\title{
Scalable Regioselective Synthesis of Rhodamine Dyes
}

\author{
Stephen J. Dwight and Sergiy Levin* \\ Promega Biosciences LLC, 277 Granada Drive, San Luis Obispo, 93401, CA, United States \\ Sergiy.Levin@promega.com
}

Supporting Information 
Table of Contents

1. General Procedures S3

2. Rhodamine Dye Structures

3. Synthetic Procedures S5

4. References $\quad$ S21

5. $\quad{ }^{1} \mathrm{H}$ and ${ }^{13} \mathrm{C}$ NMR spectra $\quad$ S22 
General. Reactions were performed using commercially obtained solvents. Unless otherwise stated, all commercially obtained reagents were used as received. Reactions were monitored by thin-layer chromatography (TLC) using EMD/Merck silica gel 60 F254 pre-coated plates $(0.25$ $\mathrm{mm})$. Flash column chromatography was performed using pre-packaged RediSep ${ }^{\circledR R f}$ columns on a CombiFlash Rf system (Teledyne ISCO Inc.). Microwave experiments were performed using a Biotage Initiator ${ }^{\circledR}$ microwave reactor. ${ }^{1} \mathrm{H}$ and ${ }^{13} \mathrm{C}$ NMR spectra were recorded on a Varian Mercury 300 (at $300 \mathrm{MHz}$ and $75 \mathrm{MHz}$ respectively) and are reported relative to internal $\mathrm{CHCl}_{3}$ $\left({ }^{1} \mathrm{H}, \delta=7.26\right), \mathrm{CDHCl}_{2}\left({ }^{1} \mathrm{H}, \delta=5.32\right), \mathrm{DMSO}_{-} d_{5}\left({ }^{1} \mathrm{H}, \delta=2.50\right), \mathrm{CD}_{2} \boldsymbol{H O D}\left({ }^{1} \mathrm{H}, \delta=3.31\right)$ and $\mathrm{CDCl}_{3}\left({ }^{13} \mathrm{C}, \delta=77.0\right), \mathrm{CD}_{2} \mathrm{Cl}_{2}\left({ }^{13} \mathrm{C}, \delta=53.8\right)$, DMSO- $d_{6}\left({ }^{13} \mathrm{C}, \delta=39.5\right), \mathrm{CD}_{3} \mathrm{OD}\left({ }^{13} \mathrm{C}, \delta=49.0\right)$. Data for ${ }^{1} \mathrm{H}$ NMR spectra are reported as follows: chemical shift $(\delta \mathrm{ppm})$ (multiplicity, coupling constant $(\mathrm{Hz})$, integration). Multiplicity and qualifier abbreviations are as follows: $\mathrm{s}=$ singlet, $\mathrm{d}=$ doublet, $\mathrm{t}=$ triplet, $\mathrm{q}=$ quartet, $\mathrm{m}=$ multiplet, $\mathrm{br}=$ broad, $\mathrm{app}=$ apparent. High resolution mass spectra were obtained on AB Sciex TripleTOF 5600+. Preparative HPLC was performed with Waters 2535 Quaternary Gradient Module utilizing XBridge PREP C18 Column $5 \mu \mathrm{m}(30 \mathrm{~mm} \mathrm{X}$ $250 \mathrm{~mm}$ ). Analytical HPLC was performed with an Agilent 1100 Series HPLC utilizing Phenomenex Synergi ${ }^{\mathrm{TM}} 2.5 \mu \mathrm{m}$ MAX-RP $100 \AA \AA$ columns $(4.6 \mathrm{~mm}$ x $50 \mathrm{~mm})$.

\section{Chemical abbreviations used:}

DMSO - dimethyl sulfoxide, DMF - $N, N$-Dimethylformamide; EtOAc - ethyl acetate; DCM dichloromethane; MeCN - acetonitrile; THF - tetrahydrofuran; TFE - 2,2,2-trifluoroethanol, NCT - non-chloro-TOM; TMR - tetramethylrhodamine; CXR - carboxy-X-rhodamine, HFIP hexafluoroisopropanol, depp - 1,3-bis(dicyclohexylphosphino)propane, NBS $-\mathrm{N}$ bromosuccinimide, AIBN - 2,2'-azobis(2-methylpropionitrile), Xantphos - 4,5Bis(diphenylphosphino)-9,9-dimethylxanthene, EtCN - propionitrile. 


\section{Rhodamine Dye Structures}<smiles></smiles>

5-carboxy-NCT (9)<smiles>CN(C)c1ccc2c(-c3ccc(C(=O)O)cc3C(=O)[O-])c3ccc(=[N+](C)C)cc-3oc2c1</smiles>

5-carboxy-TMR (16)<smiles></smiles>

5-CXR (18)<smiles></smiles>

6-CI-NCT (20)

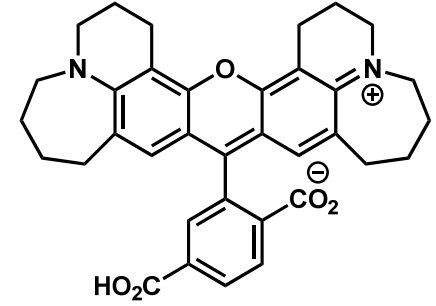

6-carboxy-NCT (10)<smiles>CN(C)c1ccc2c(-c3cc(C(=O)O)ccc3C(=O)[O-])c3ccc(=[N+](C)C)cc-3oc2c1</smiles>

6-carboxy-TMR (17)<smiles></smiles>

6-CXR (19)

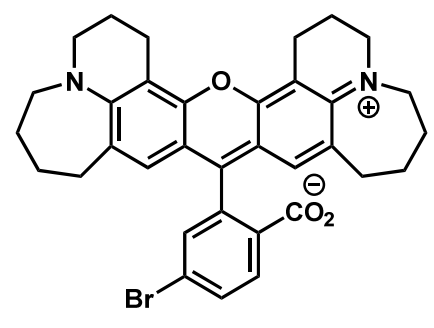

6-Br-NCT (21) 


\section{4-carboxyphthaladehydic acid (11)}

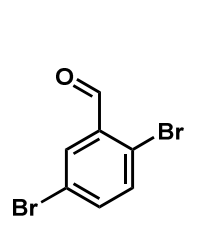

12

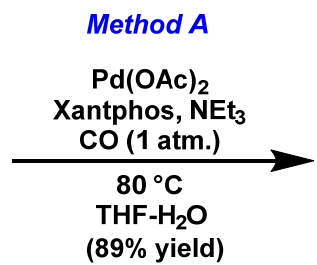

$(89 \%$ yield $)$

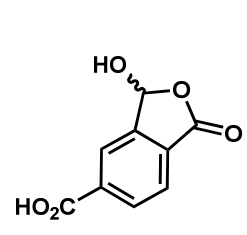

11

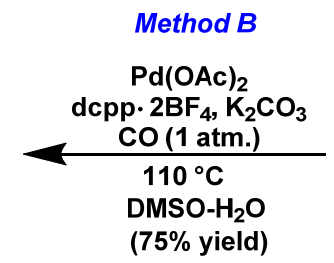

(75\% yield)

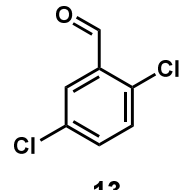

13

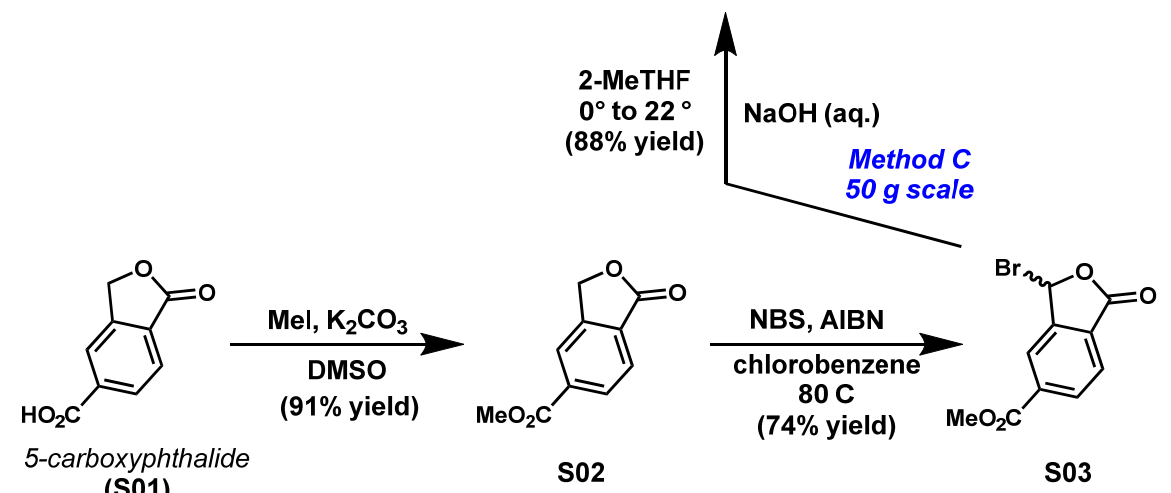

(S01)

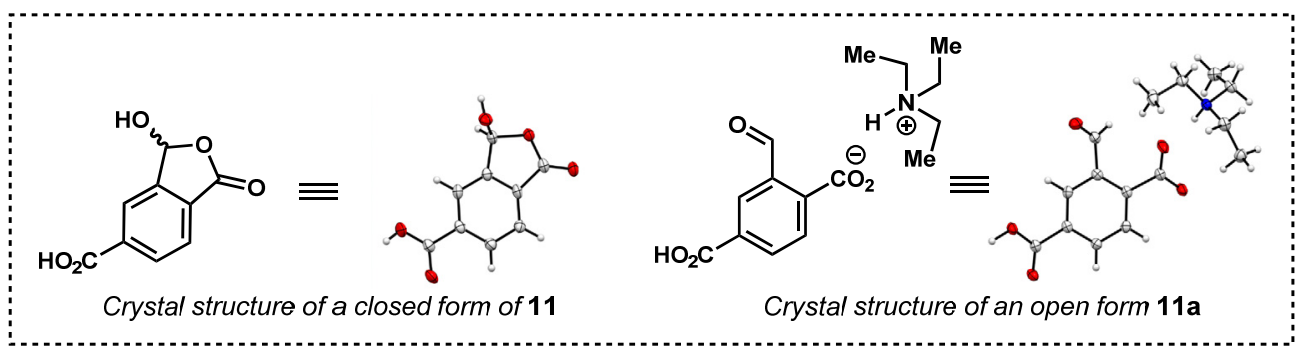

Method A: Hydroxycarbonylation of 2,5-dibromobenzaldehyde
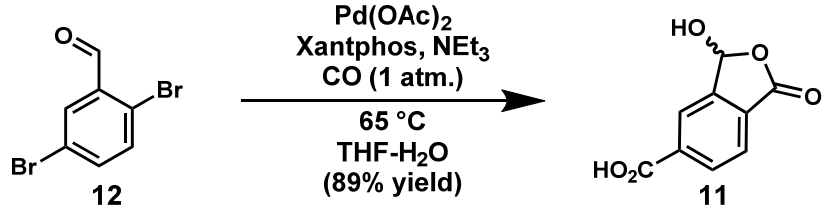

A $500 \mathrm{~mL}$ round bottom flask, equipped with stir bar and septum was charged with $\mathbf{1 2}$ (3.10 g, $11.8 \mathrm{mmol}), \mathrm{Pd}(\mathrm{OAc}) 2(132 \mathrm{mg}, 587 \mu \mathrm{mol})$ and Xantphos $(680 \mathrm{mg}, 1.17 \mathrm{mmol})$. The flask was evacuated and backfilled with argon (3 x times repeat). Degassed THF (100 mL) was added and the flask was evacuated and backfilled with carbon monoxide ( $3 \mathrm{x}$ times repeat). $\mathrm{CO}$ was allowed to bubble through the solution for 5 minutes while $\mathrm{H}_{2} \mathrm{O}(7.5 \mathrm{~mL})$ and $\mathrm{Et}_{3} \mathrm{~N}(6 \mathrm{~mL})$ was added. The resulting dark brown solution (which turns light yellow over time) was heated to 65 
${ }^{\circ} \mathrm{C}$ under $\mathrm{CO}$ balloon for 20 hours at which point HPLC analysis indicated complete consumption of the starting material. The solvent was removed in vacuo, and the residue was partitioned between DCM $(150 \mathrm{~mL})$ and water $(150 \mathrm{~mL})$. Aqueous layer $\mathrm{pH}$ was adjusted to $10-11$ with aq. $2 \mathrm{M} \mathrm{NaOH}$. Layers were separated and aqueous layer was washed $2 \times 150 \mathrm{~mL}$ DCM. Aqueous layer was acidified to $\mathrm{pH} 2$ with aq. $6 \mathrm{M} \mathrm{HCl}(6 \mathrm{M})$ and extracted with EtOAc $(3 \times 150 \mathrm{~mL})$. Organic layers were combined, dried over $\mathrm{Na}_{2} \mathrm{SO}_{4}$, filtered and concentrated to provide $2.03 \mathrm{~g}$ ( $89 \%$ yield) of 11 as white solid. ${ }^{1} \mathrm{H}$ NMR (300 MHz, DMSO- $\left.d_{6}\right) \delta 13.61(\mathrm{~s}, 1 \mathrm{H}), 8.47-8.22(\mathrm{~m}, 1 \mathrm{H}), 8.18(\mathrm{~d}$, $\mathrm{J}=7.9 \mathrm{~Hz}, 1 \mathrm{H}), 8.13(\mathrm{~s}, 1 \mathrm{H}), 7.94(\mathrm{~d}, \mathrm{~J}=7.9 \mathrm{~Hz}, 1 \mathrm{H}), 6.73(\mathrm{~s}, 1 \mathrm{H}) ;{ }^{13} \mathrm{C}$ NMR $(75 \mathrm{MHz}$, DMSO$\left.d_{6}\right) \delta 167.7,166.2,147.7,136.3,131.5,130.1,124.9,124.4,98.4$; HRMS (ESI+) calc'd for $\mathrm{C}_{9} \mathrm{H}_{7} \mathrm{O}_{5}{ }^{+}[\mathrm{M}+\mathrm{H}]^{+} 195.0293$ found 195.0285 .

Method B: Hydroxycarbonylation of 2,5-dichlorobenzaldehyde
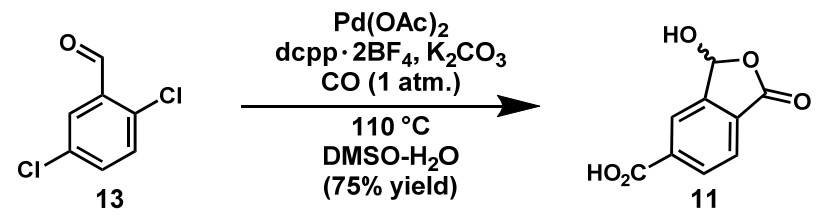

A $100 \mathrm{~mL}$ round bottom flask, equipped with stir bar and septum was charged with 13 (580 $\mathrm{mg}, 3.31 \mathrm{mmol}$ ), $\mathrm{K}_{2} \mathrm{CO}_{3}(1.37 \mathrm{~g}, 9.94 \mathrm{mmol}), \mathrm{Pd}(\mathrm{OAc})_{2}$ (37 mg, $\left.0.17 \mathrm{mmol}\right)$ and [dcpp 2BF4] (203 $\mathrm{mg}, 331 \mu \mathrm{mol})$. Flask was evacuated and backfilled with argon ( $3 \mathrm{x}$ times repeat). Degassed DMSO $(11 \mathrm{~mL})$ and $\mathrm{H}_{2} \mathrm{O}(0.25 \mathrm{~mL})$ were added and reaction vessel was evacuated and backfilled with carbon monoxide ( $3 \mathrm{x}$ times repeat). $\mathrm{CO}$ was allowed to bubble through the solution for 5 minutes. The resulting yellow suspension was heated to $110^{\circ} \mathrm{C}$ under $\mathrm{CO}$ balloon for 25 hours at which point HPLC analysis indicated complete consumption of the starting material. Reaction mixture was partitioned between DCM $(50 \mathrm{~mL})$ and water $(50 \mathrm{~mL})$. $\mathrm{pH}$ of aqueous layer was adjusted to 11 with aqueous $\mathrm{NaOH}(2 \mathrm{M})$, organic layer was separated and aqueous layer was washed with DCM (2 $\times 50 \mathrm{~mL})$. Aqueous layer was acidified to $\mathrm{pH} 2$ with aqueous $\mathrm{HCl}(6 \mathrm{M})$ and extracted with EtOAc $(3 \times 75 \mathrm{~mL})$. Organic layers were combined, dried Na2SO4, filtered and solvent was removed in vacuo. Crude yellow residue was purified by silica gel chromatography ( 0 $\rightarrow 20 \% \mathrm{MeOH} / \mathrm{DCM}$ ) to provide $0.48 \mathrm{~g}$ ( $75 \%$ yield) of 11 as light yellow solid. Analytical data matched the one reported in Method A. 
Method C: Large Scale Synthesis of 11 from 5-carboxyphthalide (S01)

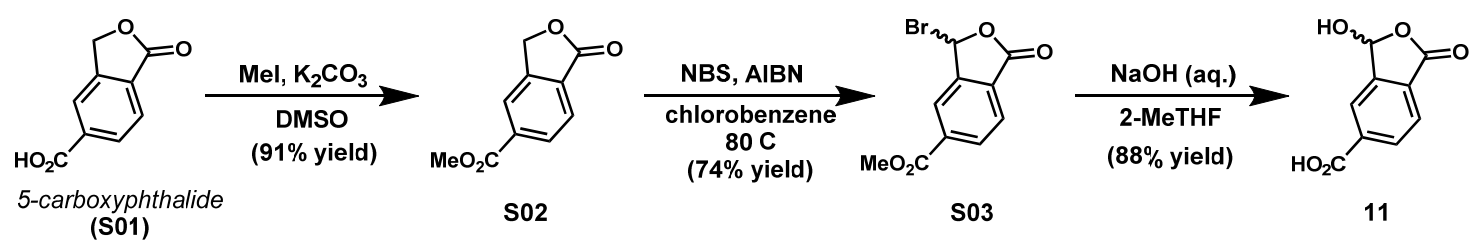

To a 2 L 3-necked round bottom flask equipped with mechanical stirrer, addition funnel and a bubbler was added 5-carboxyphthalide ( $80.0 \mathrm{~g}, 449 \mathrm{mmol})$ followed by DMSO $(800 \mathrm{~mL})$. To a vigorously stirred solution was added $\mathrm{K}_{2} \mathrm{CO}_{3}(186 \mathrm{~g}, 1.35 \mathrm{~mol})$ in portions (gas evolution!). MeI (77.0 mL, $1.24 \mathrm{~mol})$ was added slowly through addition funnel over 15 minutes. After an additional 15 minutes of stirring the reaction mixture is diluted with EtOAc (4.8 L) and water (4.8 L) and mixed well. Organic layer separated and washed with aq. $\mathrm{NaOH}(50 \mathrm{mM}, 2.4 \mathrm{~L})$. Organic layer separated and washed with water (2.4 L). Organic layer separated, diluted with DCM (0.55 L) and dried with anhydrous $\mathrm{Na}_{2} \mathrm{SO}_{4}(646 \mathrm{~g})$ for 1 hour. Solution filtered and filter cake was washed with EtOAc $(500 \mathrm{~mL})$. Combined solutions were concentrated in vacuo, to provide $78.5 \mathrm{~g}$ (91\% yield) of $\mathbf{S 0 2}$ as crystalline white solid. ${ }^{1} \mathrm{H}$ NMR (300 MHz, DMSO-d $) \delta 8.24$ (tt, J = 1.5, $0.8 \mathrm{~Hz}, 1 \mathrm{H}), 8.10(\mathrm{ddt}, \mathrm{J}=8.0,1.5,0.7 \mathrm{~Hz}, 1 \mathrm{H}), 7.96(\mathrm{ddt}, \mathrm{J}=8.0,0.8,0.7 \mathrm{~Hz}, 1 \mathrm{H}), 5.47(\mathrm{dt}, \mathrm{J}=$ 2.0, $0.8 \mathrm{~Hz}, 2 \mathrm{H}), 3.91$ (s, 3H); ${ }^{13} \mathrm{C}$ NMR (75 MHz, DMSO-d6) $\delta 169.8,165.4,147.6,134.5,129.6$, 128.8, 125.3, 124.0, 70.1, 52.7; HRMS (ESI+) calc'd for $\mathrm{C}_{10} \mathrm{H}_{9} \mathrm{O}_{4}^{+}[\mathrm{M}+\mathrm{H}]^{+} 193.0501$ found 193.0495 .

To a $5 \mathrm{~L}$ 3-necked round bottom flask equipped with mechanical stirrer, temperature probe and a bubbler was added S02 (78.5 g, $408 \mathrm{mmol})$ and NBS (72.8 g, $409 \mathrm{mmol})$ followed by chlorobenzene $(1880 \mathrm{~mL})$. After stirring for 5 minutes, AIBN (3.35 g, $20.4 \mathrm{mmol})$ was added. Reaction temperature was raised to $80^{\circ} \mathrm{C}$ and after 2 hours deep orange homogeneous mixture was allowed to cool to $22^{\circ}$. Solvent was removed in vacuo and residual solid was dissolved in DCM $(2 \mathrm{~L})$ and absorbed onto celite and purified by silica gel chromatography $(0 \rightarrow 100 \%$ $\mathrm{DCM} /$ heptane) to provide $92.7 \mathrm{~g}$ of bromide $\mathbf{S 0 3}$ as yellowish solid, which was further purified 
by trituration in $4: 1$ heptane/EtOAc $(555 \mathrm{~mL})$ at $80 \mathrm{C}$ for 1 hour, cooled to $22{ }^{\circ} \mathrm{C}$, filtered and washed with hot heptane to provide $81.7 \mathrm{~g}(74 \%)$ of $\mathbf{S 0 3}$ as white solid. ${ }^{1} \mathrm{H}$ NMR (300 MHz, $\left.\mathrm{CDCl}_{3}\right) \delta 8.45-8.17(\mathrm{~m}, 2 \mathrm{H}), 8.00(\mathrm{~d}, \mathrm{~J}=8.2 \mathrm{~Hz}, 1 \mathrm{H}), 7.43(\mathrm{~s}, 1 \mathrm{H}), 4.01(\mathrm{~s}, 3 \mathrm{H}) ;{ }^{13} \mathrm{C}$ NMR $(75$ MHz, DMSO-d6) $\delta 167.5,165.2,147.7,134.9,131.3,130.4,125.1,124.4,98.3,52.8$; HRMS $(\mathrm{ESI}+)$ calc'd for $\mathrm{C}_{10} \mathrm{H}_{8} \mathrm{BrO}_{4}{ }^{+}[\mathrm{M}+\mathrm{H}]^{+} 270.9606$ found 270.9598 .

To a 12 L 3-necked round bottom flask equipped with mechanical stirrer, addition funnel and a bubbler was added bromide $\mathbf{S 0 2}(81.7 \mathrm{~g}, 301 \mathrm{mmol})$ and 2-methyltetrahydrofuran (1.3 L). To a stirred solution was added aqueous $\mathrm{NaOH}(2.50 \mathrm{~L}, 0.60 \mathrm{M})$ over 5 minutes and the resulting biphasic solution was allowed to stir for 2 hours, at which point TLC analysis indicated consumption of the starting material. Stirring stopped, layers separated and aqueous layer was acidified with aqueous $\mathrm{HCl}$ (ca. $0.65 \mathrm{~L}, 2 \mathrm{M}$ ) to $\mathrm{pH}=1.5$ (slow addition). Acidified solution was cooled $10{ }^{\circ} \mathrm{C}$ on the ice bath and white precipitate filtered out. Filter cake was washed with cold water (ca. $1 \mathrm{~L}$ ) and filtrate dried under vacuum to provide $51.4 \mathrm{~g}$ (88\% yield) of 4carboxyphthalaldehydic acid (S11) as white solid. Analytical data matched those reported in Method A.

\section{4-chlorophthaladehydic acid (S05)}
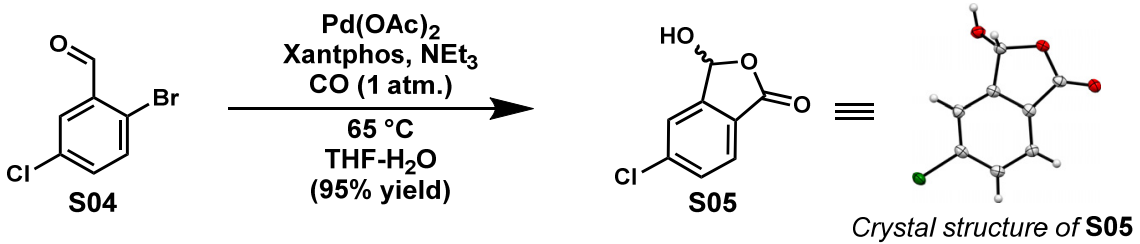

A $100 \mathrm{~mL}$ round bottom flask, equipped with stir bar and septum was charged with $\mathbf{S 0 4}$ (600 mg, $2.73 \mathrm{mmol}), \mathrm{Pd}(\mathrm{OAc})_{2}(31 \mathrm{mg}, 137 \mu \mathrm{mol})$ and Xantphos (158 mg, $\left.273 \mu \mathrm{mol}\right)$. Flask was evacuated and backfilled with argon (3 x times repeat). Degassed THF (25 mL) was added and flask was evacuated and backfilled with carbon monoxide ( $3 \mathrm{x}$ times repeat). $\mathrm{CO}$ was allowed to bubble through the solution for 5 minutes while degassed $\mathrm{H}_{2} \mathrm{O}(2.5 \mathrm{~mL})$ and degassed $\mathrm{Et}_{3} \mathrm{~N}(1.9$ $\mathrm{mL}$ ) were added. The resulting dark brown solution (which turns light yellow over time) was heated to $65{ }^{\circ} \mathrm{C}$ under $\mathrm{CO}$ balloon for 2 hours at which point HPLC analysis indicated complete 
consumption of the starting material. The solvent was removed in vacuo, and the residue was partitioned between DCM $(50 \mathrm{~mL})$ and water $(50 \mathrm{~mL})$. Aqueous layer $\mathrm{pH}$ was adjusted to $10-11$ with aqueous $\mathrm{NaOH}(2 \mathrm{M})$, if necessary. Layers were separated and aqueous layer was washed with $\operatorname{DCM}(2 \times 20 \mathrm{~mL})$. Aqueous layer acidified to $\mathrm{pH} 1-2$ with aqueous $\mathrm{HCl}(6 \mathrm{M})$ and extracted with EtOAc $(3 \times 75 \mathrm{~mL})$. Organic layers were combined, dried over $\mathrm{Na}_{2} \mathrm{SO}_{4}$, filtered and concentrated to provide $480 \mathrm{mg}$ (95\% yield) of $\mathbf{S 0 5}$ as white solid. ${ }^{1} \mathrm{H}$ NMR (300 MHz, DMSO- $\left.d 6\right) \delta 8.28$ (s, $1 \mathrm{H}), 7.85(\mathrm{~d}, J=8.1 \mathrm{~Hz}, 1 \mathrm{H}), 7.79(\mathrm{~d}, J=1.8 \mathrm{~Hz}, 1 \mathrm{H}), 7.72(\mathrm{~d}, J=8.1 \mathrm{~Hz}, 1 \mathrm{H}), 6.65(\mathrm{~s}, 1 \mathrm{H}) ;{ }^{13} \mathrm{C}$ NMR (75 MHz, DMSO-d6) $\delta 167.5,149.4,139.5,131.0,126.4,125.5,124.1,97.7$; HRMS (ESI+) calc'd for $\mathrm{C}_{8} \mathrm{H}_{6} \mathrm{ClO}_{3}{ }^{+}[\mathrm{M}+\mathrm{H}]^{+} 185.0005$ found 184.9994 .

\section{5-carboxyphthalaldehydic acid (S07)}
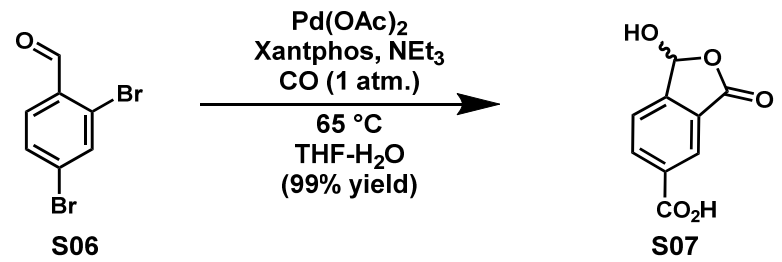

A $100 \mathrm{~mL}$ round bottom flask, equipped with stir bar and septum was charged with 2,4dibromobenzaldehyde S06 (710 mg, 2.69 mmol), Pd(OAc)2 (30.2 mg, $135 \mu \mathrm{mol})$ and Xantphos (156 mg, $269 \mu \mathrm{mol})$. Flask was evacuated and backfilled with argon (3 x times repeat). Degassed THF $(25 \mathrm{~mL})$ was added and flask was evacuated and backfilled with carbon monoxide $(3 \mathrm{x}$ times repeat). $\mathrm{CO}$ was allowed to bubble through the solution for 5 minutes while $\mathrm{H}_{2} \mathrm{O}(2.5 \mathrm{~mL})$ and $\mathrm{Et}_{3} \mathrm{~N}(1.9 \mathrm{~mL})$ was added. The resulting dark brown solution (which turns light yellow over time) was heated to $65{ }^{\circ} \mathrm{C}$ under $\mathrm{CO}$ balloon for 20 hours at which point HPLC analysis indicated complete consumption of the starting material. The solvent was removed in vacuo, and the residue was partitioned between DCM $(50 \mathrm{~mL})$ and water $(75 \mathrm{~mL})$. Aqueous layer $\mathrm{pH}$ was adjusted to 10 with $2 \mathrm{~N} \mathrm{KOH}$. Layers were separated and aqueous layer was washed with DCM (2 x $50 \mathrm{~mL})$. Aqueous layer was acidified with $1 \mathrm{M} \mathrm{HCl}$ to $\mathrm{pH} 1-2$, and extracted into EtOAc $(3 \mathrm{x} 50 \mathrm{~mL})$. Organic layers were combined, dried over $\mathrm{Na}_{2} \mathrm{SO}_{4}$ and concentrated to provide $520 \mathrm{mg}$ (99\% yield) of S07 as white solid. ${ }^{1} \mathrm{H}$ NMR (300 MHz, DMSO-d6) $\delta 13.48(\mathrm{~s}, 1 \mathrm{H}), 8.37-8.29(\mathrm{~m}, 2 \mathrm{H}), 8.23$ $(\mathrm{s}, 1 \mathrm{H}), 8.13(\mathrm{~s}, 1 \mathrm{H}), 7.80(\mathrm{~d}, \mathrm{~J}=7.9 \mathrm{~Hz}, 1 \mathrm{H}) ;{ }^{13} \mathrm{C}$ NMR $\left(75 \mathrm{MHz}, \mathrm{DMSO}-d_{6}\right) \delta 167.7,166.2$, 
147.7, 136.3, 131. 5, 130.1, 124.9, 124.4, 98.4; HRMS (ESI+) calc'd for $\mathrm{C}_{9} \mathrm{H}_{7} \mathrm{O}_{5}{ }^{+}[\mathrm{M}+\mathrm{H}]^{+}$ 195.0293 found 195.0285 .

\section{Purification of commercial 3-(dimethylamino)phenol}
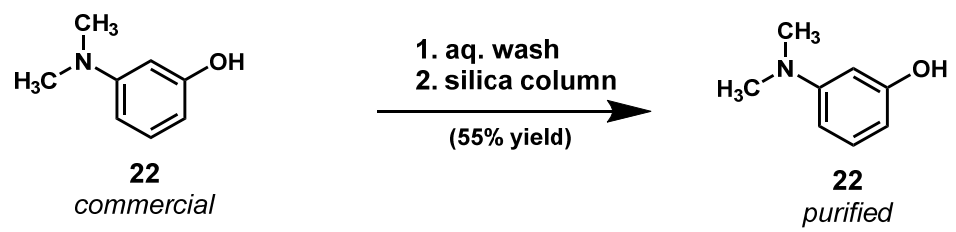

Commercial 3-(dimethylamino)phenol $(5.0 \mathrm{~g})$ is dissolved in DCM $(70 \mathrm{~mL})$ and extracted into aqueous $\mathrm{HCl}(2 \mathrm{M}, 175 \mathrm{~mL})$. Aqueous layer is washed additionally with DCM (30 mL) and $\mathrm{pH}$ adjusted to 8.3 with aqueous $\mathrm{NaOH}(6 \mathrm{M})$. Aqueous layer is extracted with $\mathrm{DCM}(3 \times 75 \mathrm{~mL})$. Organic layers are combined and dried over $\mathrm{Na}_{2} \mathrm{SO}_{4}$, solvent was removed in vacuo and residue was purified by silica gel chromatography. $(0 \rightarrow 100 \% \mathrm{DCM} /$ Heptane) to provide $2.73 \mathrm{~g}(55 \%$ mass recovery) of S06 as white crystalline solid.

\section{Synthesis of 5-carboxy-NCT (9)}
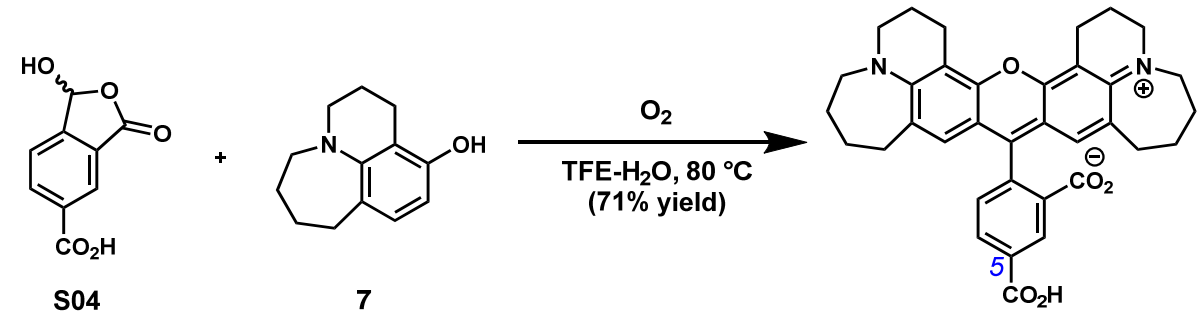

9

A $50 \mathrm{~mL}$ round-bottom flask equipped with a stir bar was charged with 5carboxyphthalaldehydic acid (S04) (140 mg, $0.721 \mathrm{mmol}$ ), phenol $7^{1}$ (367 mg, $\left.1.80 \mathrm{mmol}\right), 2,2,2-$ trifluoroethanol $(15 \mathrm{~mL})$ and water $(3 \mathrm{~mL})$. Oxygen was allowed to bubble through the solution for 5 minutes. The resulting yellow solution was gently warmed under oxygen atmosphere to 80 ${ }^{\circ} \mathrm{C}$ and stirred vigorously for 24 hours. Solvent was removed in vacuo, and dark blue residue was purified by silica gel chromatography $(0 \rightarrow 60 \% \mathrm{MeOH} / \mathrm{DCM}$ to provide $290 \mathrm{mg}$ (71\% yield) of 5-carboxy-NCT (9) as dark blue solid. ${ }^{1} \mathrm{H}$ NMR $\left(300 \mathrm{MHz}, \mathrm{DMSO}-d_{6}\right) \delta 8.40(\mathrm{dd}, J=1.5,0.7 \mathrm{~Hz}$, $1 \mathrm{H}), 8.27(\mathrm{dd}, J=8.1,1.4 \mathrm{~Hz}, 1 \mathrm{H}), 7.37(\mathrm{dd}, J=8.0,0.7 \mathrm{~Hz}, 1 \mathrm{H}), 6.26(\mathrm{~s}, 2 \mathrm{H}), 3.19-3.12(\mathrm{~m}$, 
4H), $3.05-3.00(\mathrm{~m}, 4 \mathrm{H}), 2.93-2.87(\mathrm{~m}, 4 \mathrm{H}), 2.52(\mathrm{~m}, 4 \mathrm{H}$, overlap with DMSO-d $d), 1.86-1.80$ (m, 4H), $1.71-1.65(\mathrm{~m}, 4 \mathrm{H}), 1.49-1.41(\mathrm{~m}, 4 \mathrm{H}) ;{ }^{13} \mathrm{C}$ NMR (75 MHz, DMSO-d6) $\delta$ 167.9, 166.1, 155.6, 150.2, 147.3, 135.8, 133.1, 129.9, 127.0, 125.4, 125.0, 124.6, 114.2, 108.5, 56.0, 53.1, 33.5, 29.1, 25.2, 21.7, 18.2; HRMS (ESI+) calc'd for $\mathrm{C}_{35} \mathrm{H}_{35} \mathrm{~N}_{2} \mathrm{O}_{5}^{+}[\mathrm{M}+\mathrm{H}]^{+} 563.2540$ found 563.2541 .

\section{Synthesis of 6-carboxy-NCT (10)}

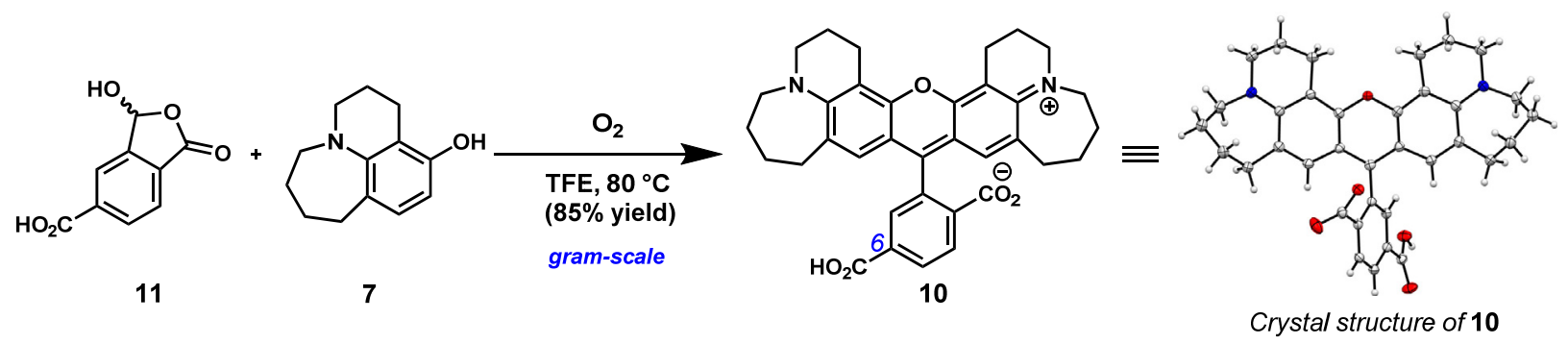

To a $500 \mathrm{~mL}$ round bottom flask, equipped with septa and stir bar was added $11(1.00 \mathrm{~g}$, $5.15 \mathrm{mmol})$ and $7^{1}(2.50 \mathrm{~g}, 12.3 \mathrm{mmol})$. Flask was sparged with $\mathrm{O}_{2}$ and oxygen saturated TFE (100 $\mathrm{mL}$ ) was added. Two large oxygen balloons were attached through septa. The reaction was heated to $80{ }^{\circ} \mathrm{C}$ for 48 hours, at which point HPLC analysis indicated complete consumption of the starting materials. Solvent was removed in vacuo and purified by silica gel chromatography $(0 \rightarrow$ $40 \% \mathrm{MeOH} / \mathrm{DCM})$ to provide $2.90 \mathrm{~g}(85 \%)$ of 6-carboxy-NCT (10) as a dark blue solid. ${ }^{1} \mathrm{H}$ NMR $\left(300 \mathrm{MHz}, \mathrm{DMSO}-d_{6}\right) \delta 8.21(\mathrm{dd}, J=8.0,1.3 \mathrm{~Hz}, 1 \mathrm{H}), 8.09(\mathrm{dd}, J=8.0,0.6 \mathrm{~Hz}, 1 \mathrm{H}), 7.61(\mathrm{dd}, J$ $=1.2,0.7 \mathrm{~Hz}, 1 \mathrm{H}), 6.27(\mathrm{~s}, 2 \mathrm{H}), 3.20-3.13(\mathrm{~s}, 4 \mathrm{H}), 3.07-3.01(\mathrm{~m}, 4 \mathrm{H}), 2.89(\mathrm{t}, J=6.5 \mathrm{~Hz}, 4 \mathrm{H})$, $2.52\left(\mathrm{~m}, 4 \mathrm{H}\right.$, overlap with DMSO- $\left.d_{5}\right), 1.87-1.77(\mathrm{~m}, 4 \mathrm{H}), 1.71-1.62(\mathrm{~m}, 4 \mathrm{H}), 1.49-1.40(\mathrm{~m}$, $4 \mathrm{H}) ;{ }^{13} \mathrm{C}$ NMR (75 MHz, DMSO- $\left.d_{6}\right) \delta 166.0,165.7,155.62,154.3,151.1,134.7,134.1,133.7$, 131.4, 131.0, 130.8, 129.9, 128.3, 113.3, 108.6, 52.9, 52.4, 31.8, 25.2, 23.5, 20.2, 20.0; HRMS (ESI+) calc'd for $\mathrm{C}_{35} \mathrm{H}_{35} \mathrm{~N}_{2} \mathrm{O}_{5}{ }^{+}[\mathrm{M}+\mathrm{H}]^{+} 563.2540$ found 563.2540. 


\section{Synthesis of 5-Carboxytetramethylrhodamine (16)}

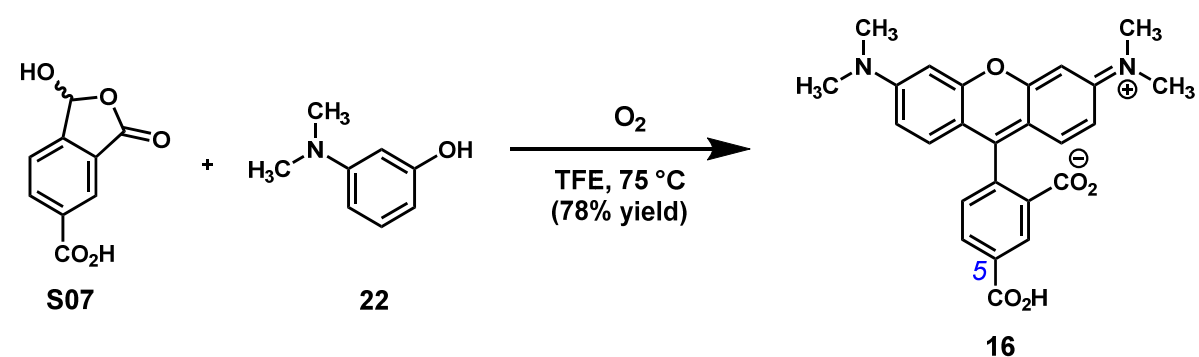

A $50 \mathrm{~mL}$ round-bottom flask equipped with stir bar was charged with 5carboxyphthalaldehydic acid (S04) (84.0 mg, $0.43 \mathrm{mmol}), 3$-(dimethylamino)phenol (22) (237 mg, $1.73 \mathrm{mmol})$, and trifluoroethanol $(20 \mathrm{~mL})$. Oxygen was allowed to bubble through the solution for 5 minutes. The resulting dark brown solution was gently warmed up under oxygen atmosphere to $75^{\circ} \mathrm{C}$ and stirred vigorously for 22 hours. The solvent was concentrated in vacuo, and dark blue residue was purified by silica gel chromatography $(0 \rightarrow 60 \% \mathrm{MeOH} / \mathrm{DCM}$ to provide $146 \mathrm{mg}$ (78\% yield) of 5-carboxy-TMR (16) as a dark blue solid. ${ }^{1} \mathrm{H}$ NMR (300 MHz, DMSO- $\left.d 6\right) 8.38$ (s, 1H), $8.27(\mathrm{~d}, J=7.8 \mathrm{~Hz}, 1 \mathrm{H}), 7.14(\mathrm{~d}, J=7.9 \mathrm{~Hz}, 1 \mathrm{H}), 6.53-6.45(\mathrm{~m}, 6 \mathrm{H}), 2.93(\mathrm{~s}, 12 \mathrm{H}) ;{ }^{13} \mathrm{C}$ NMR (75 MHz, DMSO-d6) $\delta$ 169.0, 167.6, 153.6, 152.1, 151.9, 140.5, 136.0, 128.4, 126.3, 124.6, 123.1, 108.9, 106.1, 98.0, 84.3, 39.8 (overlap with DMSO-d6); HRMS (ESI+) calc'd for $\mathrm{C}_{25} \mathrm{H}_{23} \mathrm{~N}_{2} \mathrm{O}_{5}^{+}[\mathrm{M}+\mathrm{H}]^{+} 431.1601$ found 431.1601 .

\section{Synthesis of 6-carboxytetramethylrhodamine (17)}

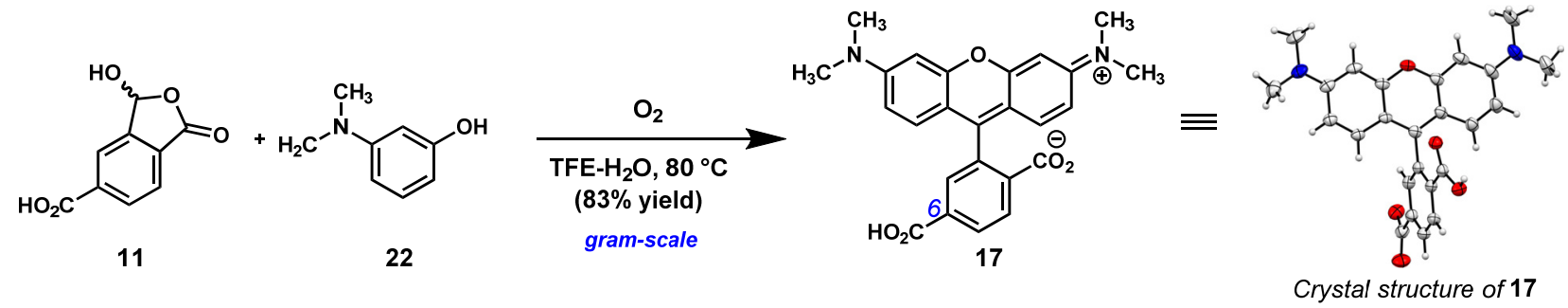

To a $500 \mathrm{~mL}$ round bottom flask, equipped with septa and stir bar was added 11 (1.00 g, $5.15 \mathrm{mmol})$ and purified phenol $22(2.12 \mathrm{~g}, 15.45 \mathrm{mmol})$. Flask was sparged with $\mathrm{O}_{2}$ and oxygen saturated TFE $(80 \mathrm{~mL})$ and water $(20 \mathrm{~mL})$ were added. Two large oxygen balloons (ca $\left.8 \mathrm{~L} \mathrm{of}_{2}\right)$ were attached through septa. The reaction was heated to $80^{\circ} \mathrm{C}$ for 48 hours, at which point HPLC 
analysis indicated complete consumption of the starting material. Solvent was removed in vacuo and purified by silica gel chromatography $(0 \rightarrow 60 \% \mathrm{MeOH} / \mathrm{DCM})$ to provide $1.83 \mathrm{~g}(83 \%)$ of 6carboxy-TMR (14) as a dark blue solid. ${ }^{1} \mathrm{H}$ NMR $\left(300 \mathrm{MHz}\right.$, Methanol- $\left.d_{4}\right) \delta 8.40(\mathrm{dd}, J=8.2,0.7$ $\mathrm{Hz}, 1 \mathrm{H}), 8.36(\mathrm{dd}, J=8.2,1.5 \mathrm{~Hz}, 1 \mathrm{H}), 7.95(\mathrm{dd}, J=1.6,0.7 \mathrm{~Hz}, 1 \mathrm{H}), 7.12(\mathrm{~d}, J=9.5 \mathrm{~Hz}, 2 \mathrm{H})$, $7.03(\mathrm{dd}, J=9.5,2.4 \mathrm{~Hz}, 2 \mathrm{H}), 6.95(\mathrm{~d}, J=2.4 \mathrm{~Hz}, 2 \mathrm{H}), 3.28$ (s, $12 \mathrm{H}$, overlap with $\left.\mathrm{CD}_{2} \mathrm{HOD}\right) ;{ }^{13} \mathrm{C}$ NMR (75 MHz, Methanol- $\left.d_{4}\right) \delta$ 166.3, 165.9, 159.0, 157.7, 157.5, 134.6, 134.5, 134.0, 131.4, 130.9, 130.9, 130.5, 114.2, 113.5, 96.1, 39.5; HRMS (ESI+) calc'd for $\mathrm{C}_{25} \mathrm{H}_{23} \mathrm{~N}_{2} \mathrm{O}_{5}{ }^{+}[\mathrm{M}+\mathrm{H}]^{+}$ 431.1601 found 431.1607.

\section{Synthesis of 5-carboxy-X-rhodamine (18)}

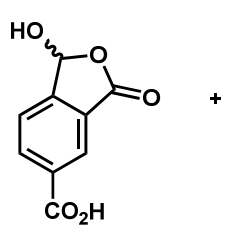

S07

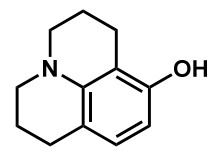

S09

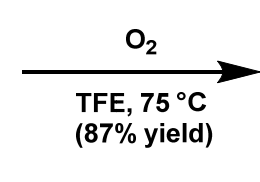

quipped with stir bar, 5-carboxyphthalaldehydic acid (S04) (116 mg, $0.60 \mathrm{mmol})$, phenol $\mathbf{S 0 9}(238 \mathrm{mg}, 1.25 \mathrm{mmol})$ and trifluoroethanol $(25 \mathrm{~mL})$. Oxygen was allowed to bubble through the solution for 5 minutes. The resulting dark brown solution was gently warmed up under oxygen atmosphere to $75^{\circ} \mathrm{C}$ and stirred vigorously for 21 hours. The solvent was removed in vacuo, and dark blue residue was purified by silica gel chromatography $(0 \rightarrow 60 \% \mathrm{MeOH} / \mathrm{DCM}$ to provide $279 \mathrm{mg}$ (87\% yield) of 5-CXR (18) as a dark blue solid. ${ }^{1} \mathrm{H}$ NMR (300 MHz, DMSO-d $\left.d\right) \delta 8.43(\mathrm{~s}, 1 \mathrm{H}), 8.19(\mathrm{dd}, \mathrm{J}=7.9,1.6 \mathrm{~Hz}, 1 \mathrm{H}), 7.27(\mathrm{~d}$, $\mathrm{J}=8.0 \mathrm{~Hz}, 1 \mathrm{H}), 6.21(\mathrm{~s}, 2 \mathrm{H}), 3.30-3.16(\mathrm{~m}, 8 \mathrm{H}), 2.90(\mathrm{t}, \mathrm{J}=6.6 \mathrm{~Hz}, 4 \mathrm{H}), 2.02-1.91(\mathrm{~m}, 4 \mathrm{H})$, $1.86-1.72(\mathrm{~m}, 4 \mathrm{H}) ;{ }^{13} \mathrm{C}$ NMR $\left(75 \mathrm{MHz}, \mathrm{CD}_{2} \mathrm{Cl}_{2}\right.$-TFIP- $\left.d_{2}\right) \delta 174.0,171.2,155.9,153.4,152.6$, 139.4, 135.90, 134.1, 133.4, 132.9, 132.1, 126.8, 125.5, 113.7, 106.6, 51.9, 51.4, 28.2, 21.4, 20.8, 20.5; HRMS (ESI+) calc'd for $\mathrm{C}_{33} \mathrm{H}_{31} \mathrm{~N}_{2} \mathrm{O}_{5}{ }^{+}[\mathrm{M}+\mathrm{H}]^{+} 535.2227$ found 535.2228. 


\section{Synthesis of 6-carboxy-X-rhodamine (19)}

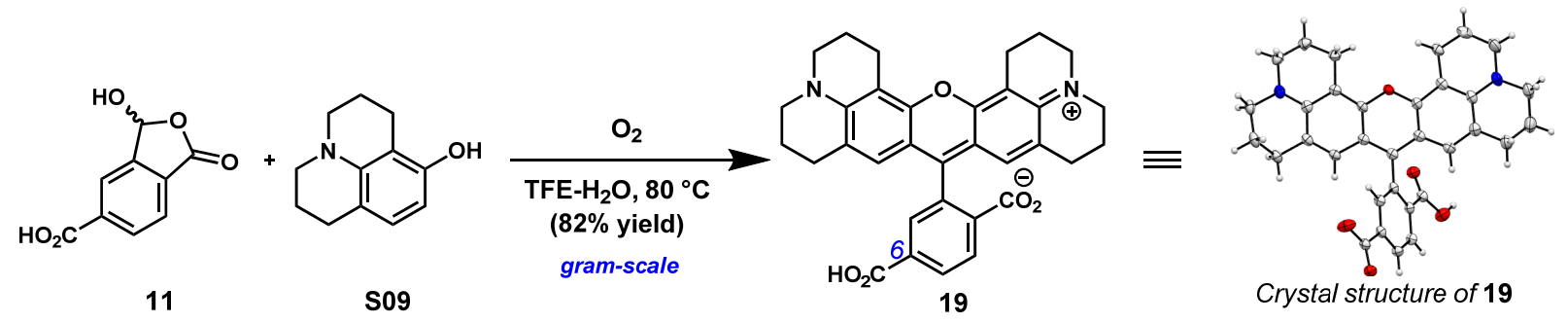

To a $500 \mathrm{~mL}$ round bottom flask, equipped with septa and stir bar was added 11 (1.00 g, $5.15 \mathrm{mmol}$ ) and phenol S09 $(2.24 \mathrm{~g}, 11.9 \mathrm{mmol})$. Flask was sparged with $\mathrm{O}_{2}$ and oxygen saturated TFE $(80 \mathrm{~mL})$ and water $(20 \mathrm{~mL})$ were added. Two large oxygen balloons (ca $8 \mathrm{~L}$ of $\mathrm{O}_{2}$ ) were attached through septa. The reaction was heated to $80{ }^{\circ} \mathrm{C}$ for 20 hours, at which point HPLC analysis indicated complete consumption of the starting material. Solvent was removed in vacuo and purified by silica gel chromatography $(0 \rightarrow 50 \% \mathrm{MeOH} / \mathrm{DCM})$ to provide $2.27 \mathrm{~g}(82 \%)$ of 6CXR (19) as a dark blue-purple solid. ${ }^{1} \mathrm{H}$ NMR (300 MHz, $\mathrm{CD}_{2} \mathrm{Cl}_{2}$-TFIP- $\left.d_{2}\right) \delta 8.26$ (s, 2H), 7.83 (s, 1H), $6.68(\mathrm{~s}, 2 \mathrm{H}), 3.54-3.43(\mathrm{~m}, 8 \mathrm{H}), 3.11(\mathrm{t}, J=6.4 \mathrm{~Hz}, 4 \mathrm{H}), 2.71(\mathrm{t}, J=6.1 \mathrm{~Hz}, 4 \mathrm{H}), 2.15$ $(\mathrm{p}, J=6.3 \mathrm{~Hz}, 4 \mathrm{H}) ;{ }^{13} \mathrm{C} \mathrm{NMR}\left(75 \mathrm{MHz}, \mathrm{CD}_{2} \mathrm{Cl}_{2}\right.$-TFIP- $\left.d_{2}\right) \delta 173.8,171.9,155.9,153.5,152.6$, 137.4, 137.0, 135.6, 132.5, 132.2, 131.8, 126.9, 125.6, 114.0, 106.7, 51.9, 51.4, 28.3, 21.4, 20.8, 20.56; HRMS (ESI+) calc'd for $\mathrm{C}_{33} \mathrm{H}_{31} \mathrm{~N}_{2} \mathrm{O}_{5}{ }^{+}[\mathrm{M}+\mathrm{H}]^{+} 535.2227$ found 535.2228.

\section{Synthesis of 6-Cl-NCT (20)}

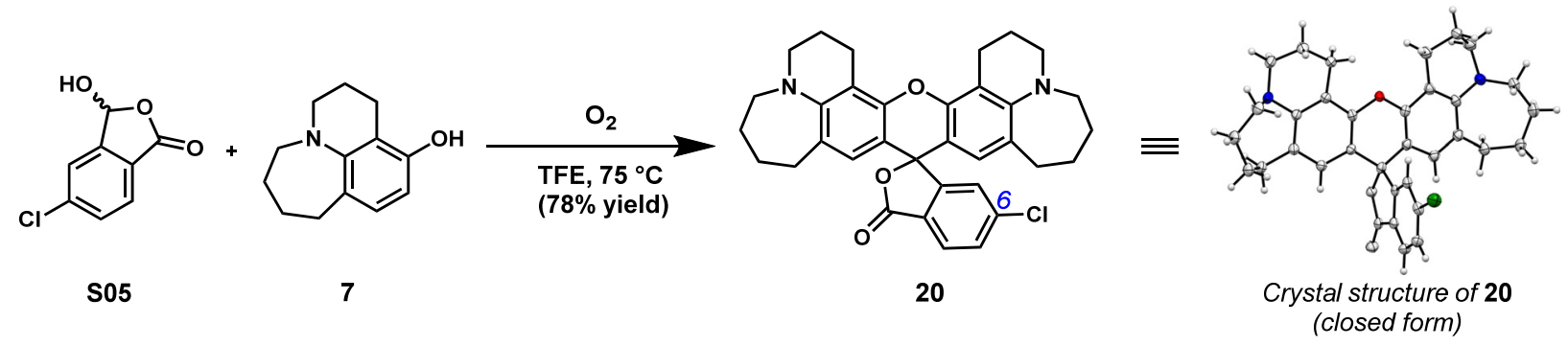

A $50 \mathrm{~mL}$ round-bottom flask equipped with stir bar was charged with 4chlorophthalaldehydic acid (S06) $(85.0 \mathrm{mg}, 0.46 \mathrm{mmol})$, phenol 7 (197 mg, $0.97 \mathrm{mmol}$ ), and trifluoroethanol $(20 \mathrm{~mL})$. Oxygen was allowed to bubble through the solution for 5 minutes. The 
resulting clear yellow solution was gently warmed up under oxygen atmosphere to $75^{\circ} \mathrm{C}$. A deep red color appeared after 10 minutes, and the resulting colored solution was stirred vigorously for 18 hours. The solvent was evaporated in vacuo, and dark blue residue was purified by silica gel chromatography $(0 \rightarrow 60 \% \mathrm{MeOH} / \mathrm{DCM}$ to provide $200 \mathrm{mg}$ (78\% yield) of $\mathbf{2 0}$ as a dark blue solid. ${ }^{1} \mathrm{H}$ NMR (300 MHz, DMSO-d6) $\delta 7.99(\mathrm{dd}, J=8.2,0.6 \mathrm{~Hz}, 1 \mathrm{H}), 7.75(\mathrm{dd}, J=8.2,1.8 \mathrm{~Hz}, 1 \mathrm{H})$, $7.43(\mathrm{dd}, J=1.8,0.6 \mathrm{~Hz}, 1 \mathrm{H}), 6.26(\mathrm{~s}, 2 \mathrm{H}), 3.15(\mathrm{~m}, 4 \mathrm{H}), 2.88(\mathrm{~m}, 4 \mathrm{H}), 2.54(\mathrm{~m}, 4 \mathrm{H}), 1.83(\mathrm{~m}$, 4H), $1.46(\mathrm{~m}, 4 \mathrm{H}) ;{ }^{13} \mathrm{C}$ NMR (75 MHz, DMSO-d6) $\delta 167.8,154.2,150.3,147.3,140.4,130.6$, $130.2,126.5,125.4,125.0,124.2,114.4,108.4,84.3,56.2,53.2,33.7,29.3,25.3,21.8,18.2$; HRMS (ESI+) calc'd for $\mathrm{C}_{34} \mathrm{H}_{34} \mathrm{ClN}_{2} \mathrm{O}_{3}^{+}[\mathrm{M}+\mathrm{H}]^{+} 553.2252$ found 553.2251.

\section{Synthesis of 6-Br-NCT (21)}
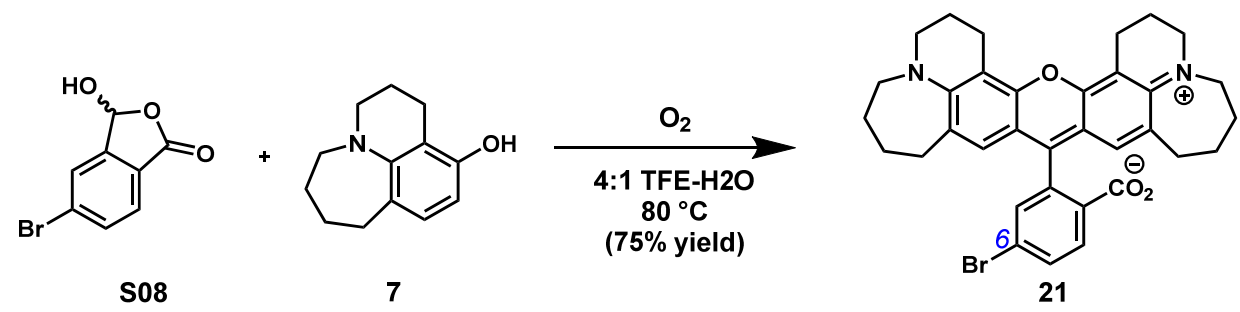

A $50 \mathrm{~mL}$ round-bottom flask equipped with stir bar was charged with 4bromophthalaldehydic acid ${ }^{2}$ (S08) $(75.0 \mathrm{mg}, 328 \mu \mathrm{mol})$, phenol $7^{1}$ (140 mg, $\left.688 \mu \mathrm{mol}\right)$, water (4 $\mathrm{mL})$, and trifluoroethanol $(16 \mathrm{~mL})$. Oxygen was allowed to bubble through the solution for 5 minutes. The resulting clear yellow solution was gently warmed up under oxygen atmosphere to $80^{\circ} \mathrm{C}$. The resulting solution was stirred vigorously for 20 hours. Solvent was evaporated in vacuo, and dark blue residue was purified by silica gel chromatography $(0 \rightarrow 50 \% \mathrm{MeOH} / \mathrm{DCM}$ to provide $147 \mathrm{mg}$ (75\% yield) of 21 as a dark blue solid. ${ }^{1} \mathrm{H}$ NMR $\left(300 \mathrm{MHz}, \mathrm{CD}_{2} \mathrm{Cl}_{2}\right) \delta 7.85(\mathrm{~d}, \mathrm{~J}$ $=8.1 \mathrm{~Hz}, 1 \mathrm{H}), 7.73(\mathrm{dd}, \mathrm{J}=8.2,1.6 \mathrm{~Hz}, 1 \mathrm{H}), 6.31(\mathrm{~s}, 2 \mathrm{H}), 3.28-3.13(\mathrm{~m}, 4 \mathrm{H}), 3.12-3.02(\mathrm{~m}$, $4 \mathrm{H}), 3.01-3.84(\mathrm{~m}, 4 \mathrm{H}), 2.66-2.52(\mathrm{~m}, 4 \mathrm{H}), 1.99-1.81(\mathrm{~m}, 4 \mathrm{H}), 1.80-1.69(\mathrm{~m}, 4 \mathrm{H}), 1.62-$ $1.43(\mathrm{~m}, 4 \mathrm{H}) ;{ }^{13} \mathrm{C} \mathrm{NMR}\left(75 \mathrm{MHz}, \mathrm{CD}_{2} \mathrm{Cl}_{2}\right) \delta 168.9,154.9,151.1,148.5,133.2,130.9,129.9$, 127.9, 126.8, 126.6, 125.8, 115.1, 109.1, 57.2, 54.4 (overlap with $\mathrm{CD}_{2} \mathrm{Cl}_{2}$ ), 34.7, 30.0, 26.0, 22.6, 19.2; HRMS (ESI+) calc'd for $\mathrm{C}_{34} \mathrm{H}_{34} \mathrm{BrN}_{2} \mathrm{O}^{+}[\mathrm{M}+\mathrm{H}]^{+} 597.1747$ found 597.1735. 


\section{Synthesis of triarylmethane 24}

\section{Unoptimized reaction}

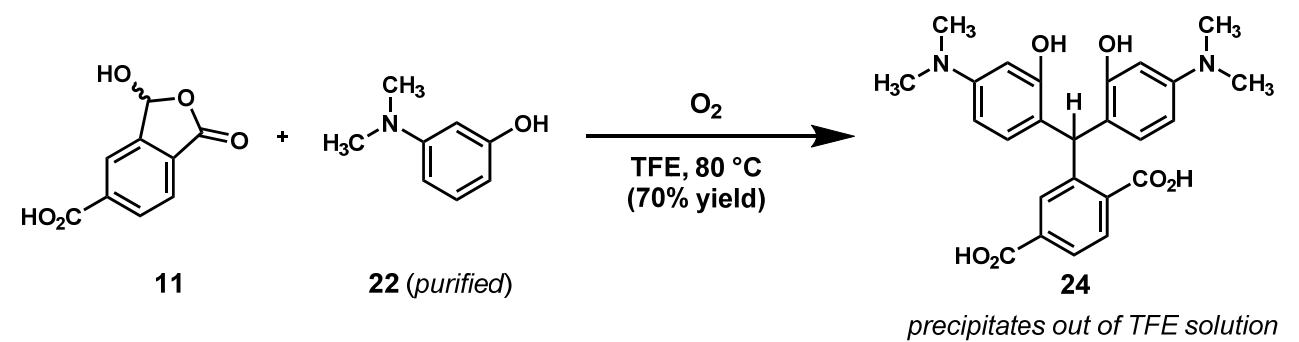

To a $50 \mathrm{~mL}$ round bottom flask, equipped with septa and stir bar was added 11 (106 mg, $547 \mu \mathrm{mol})$ and purified phenol $22(302 \mathrm{~g}, 2.20 \mathrm{mmol})$. Flask was sparged with $\mathrm{O}_{2}$ and TFE (10 $\mathrm{mL}$ ) was added. An oxygen balloon was attached and the reaction was heated to $80{ }^{\circ} \mathrm{C}$ for 16 hours, at which point reaction was cooled to $22^{\circ} \mathrm{C}$ and white precipitate was filtered out to provide $172 \mathrm{mg}\left(70 \%\right.$ ) of 24 as pinkish solid. ${ }^{1} \mathrm{H}$ NMR (400 MHz, DMSO- $\left.d 6\right) \delta 12.83$ (br. s, 2H), 8.77 (br. s, 2H), 7.76 (dd, J = 8.0, 1.7 Hz, 1H), 7.69 (d, J = 8.0 Hz, 1H), 7.55 (d, J = 1.6 Hz, 1H), 6.52 (s, 1H), 6.29 (d, J = 8.4 Hz, 2H), $6.14(\mathrm{~d}, \mathrm{~J}=2.5 \mathrm{~Hz}, 2 \mathrm{H}), 6.05(\mathrm{dd}, \mathrm{J}=8.5,2.5 \mathrm{~Hz}, 2 \mathrm{H}), 2.80$ (s, $12 \mathrm{H}) ;{ }^{13} \mathrm{C}$ NMR (75 MHz, DMSO- $\left.d_{6}\right) \delta 168.8,167.1,155.3,149.8,145.6,136.5,131.6,130.7$, 129.7, 129.33, 126.1, 119.0, 103.2, 99.8, 40.2; HRMS (ESI+) calc'd for $\mathrm{C}_{25} \mathrm{H}_{27} \mathrm{~N}_{2} \mathrm{O}_{6}{ }^{+}[\mathrm{M}+\mathrm{H}]^{+}$ 451.1869 found 451.1863 .

\section{Synthesis of triarylmethane 15}

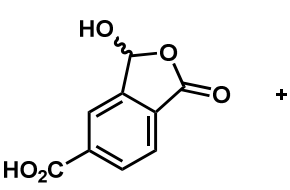

11

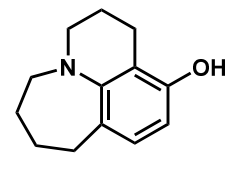

7

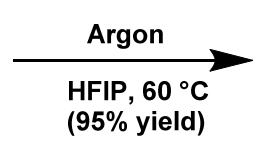

(95\% yield)

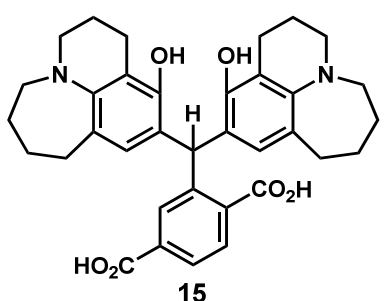

To a $25 \mathrm{~mL}$ round bottom flask, equipped with septa and stir bar was added 11 (65 mg, $335 \mu \mathrm{mol})$ and phenol $7^{1}(170 \mathrm{mg}, 837 \mu \mathrm{mol})$ and degassed HFIP $(5 \mathrm{~mL})$. The reaction was heated under agron to $80^{\circ} \mathrm{C}$ for 26 hours, at which point heating stopped and solvent was removed in vacuo. Crude blue residue was purified by silica gel chromatography $(0 \rightarrow 70 \% \mathrm{MeOH} / \mathrm{DCM}$ to 
provide $120 \mathrm{mg}$ (95\% yield) of 15 as a blue solid. ${ }^{1} \mathrm{H}$ NMR (300 MHz, DMSO-d 6 ) $\delta 7.73(\mathrm{~s}, 1 \mathrm{H})$, $7.73-7.67(\mathrm{~m}, 1 \mathrm{H}$, overlap with previous peak), $7.57(\mathrm{~d}, \mathrm{~J}=8.4 \mathrm{~Hz}, 1 \mathrm{H}), 3.07-2.93(\mathrm{~m}, 4 \mathrm{H})$, $2.92-2.75(\mathrm{~m}, 4 \mathrm{H}), 2.47-2.36(\mathrm{~m}, 4 \mathrm{H}$, overlap with DMSO-ds), $1.71-1.55(\mathrm{~m}, 8 \mathrm{H}), 1.50-1.23$ (m, 4H); ${ }^{13} \mathrm{C}$ NMR (75 MHz, DMSO- $\left.d_{6}\right) \delta$ 171.0, 167.2, 151.0, 146.9, 141.8, 132.0, 130.0, 128.6, 126.92, 126.2, 125.0, 122.3, 114.6, 56.9, 53.8, 34.7, 30.5, 26.3, 22.7, 18.0; HRMS (ESI+) calc'd for $\mathrm{C}_{35} \mathrm{H}_{39} \mathrm{~N}_{2} \mathrm{O}_{6}{ }^{+}[\mathrm{M}+\mathrm{H}]^{+} 583.2808$ found 583.2802.

\section{Synthesis of lactone 14}

Unoptimized reaction

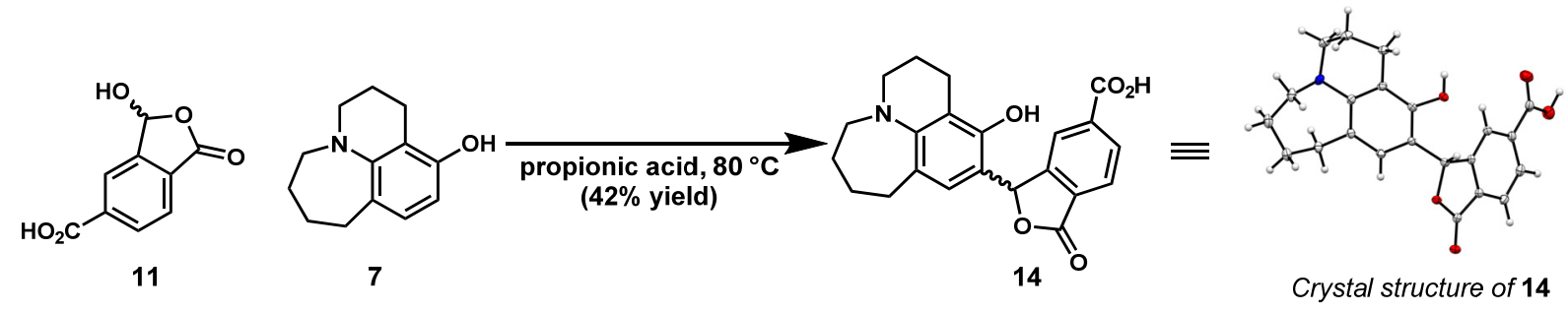

To a $50 \mathrm{~mL}$ round bottom flask equipped with stir bar was added 4carboxyphthalaldehydic acid $(26.0 \mathrm{mg}, 134 \mu \mathrm{mol})$ and $7^{1}(68.1 \mathrm{mg}, 335 \mu \mathrm{mol})$, followed by propionic acid $(5 \mathrm{~mL})$. The resulting stirred solution was heated to $80{ }^{\circ} \mathrm{C}$ open to air for 20 hours, at which point solution was concentrated and crude residue was purified by silica gel chromatography $\left(0 \rightarrow 10 \% \mathrm{MeOH} / \mathrm{DCM}\right.$ to provide $21.3 \mathrm{mg}$ ( $42 \%$ yield) of 14 as white solid. ${ }^{1} \mathrm{H}$ NMR (300 MHz, DMSO-d6) $\delta 13.51$ (s, 1H), $8.42(\mathrm{~s}, 1 \mathrm{H}), 8.11(\mathrm{dd}, \mathrm{J}=8.3,1.3 \mathrm{~Hz}, 1 \mathrm{H}), 7.97$ (d, $\mathrm{J}=8.0 \mathrm{~Hz}, 1 \mathrm{H}), 7.90(\mathrm{~s}, 1 \mathrm{H}), 6.81(\mathrm{~s}, 1 \mathrm{H}), 6.56(\mathrm{~s}, 1 \mathrm{H}), 3.09-3.98(\mathrm{~m}, 2 \mathrm{H}), 2.95-2.87(\mathrm{~m}, 2 \mathrm{H})$, $2.61-2.52(\mathrm{~m}, 4 \mathrm{H}$, overlap with DMSO- $d 5), 1.75-1.59(\mathrm{~m}, 4 \mathrm{H}), 1.47-1.37(\mathrm{~m}, 2 \mathrm{H}) ;{ }^{13} \mathrm{C} \mathrm{NMR}$ (75 MHz, DMSO-d6) $\delta 169.6,166.4,151.4,151.0,150.0,136.0,129.8,129.1,126.2,125.0,123.6$, 115.8, 114.2, 79.8, 56.5, 53.2, 33.9, 29.7, 25.6, 22.4, 18.3; HRMS (ESI+) calc'd for $\mathrm{C}_{22} \mathrm{H}_{22} \mathrm{NO}_{5}{ }^{+}$ $[\mathrm{M}+\mathrm{H}]^{+} 380.1498$ found 380.1483 .

\section{Synthesis of lactone S09}

Unoptimized reaction 


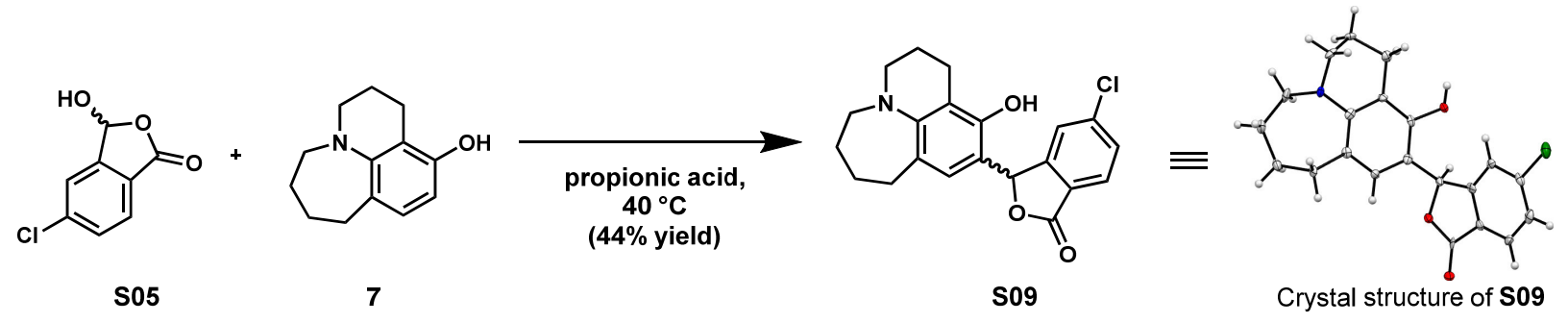

To a $100 \mathrm{~mL}$ round bottom flask equipped with stir bar was added 4chlorophthalaldehydic acid (264 mg, $1.43 \mathrm{mmol})$ and $7^{1}(320 \mathrm{mg}, 157 \mathrm{mmol})$, followed by propionic acid $(25 \mathrm{~mL})$. The resulting stirred solution was heated to $40{ }^{\circ} \mathrm{C}$ under argon for 20 hours, at which point HPLC analysis indicated consumption of the starting material. Solvent was removed in vacuo and crude residue was purified by silica gel chromatography $(0 \rightarrow 50 \%$ EtOAc/heptane to provide $230 \mathrm{mg}$ (44\% yield) of S09 as white solid. ${ }^{1} \mathrm{H}$ NMR (300 MHz, DMSOd6) $\delta 8.40(\mathrm{~s}, 1 \mathrm{H}), 7.88(\mathrm{~d}, \mathrm{~J}=8.1 \mathrm{~Hz}, 1 \mathrm{H}), 7.64(\mathrm{dd}, \mathrm{J}=8.1,1.4 \mathrm{~Hz}, 1 \mathrm{H}), 7.52(\mathrm{~d}, \mathrm{~J}=1.4 \mathrm{~Hz}, 1 \mathrm{H})$, $6.74(\mathrm{~s}, 1 \mathrm{H}), 6.53(\mathrm{~s}, 1 \mathrm{H}), 3.06-3.02(\mathrm{~m}, 2 \mathrm{H}), 2.95-2.89(\mathrm{~m}, 2 \mathrm{H}), 2.58-2.52(\mathrm{~m}, 4 \mathrm{H}$, overlap with DMSO-d5), 1.74 - $1.61(\mathrm{~m}, 4 \mathrm{H}), 1.50$ - $1.39(\mathrm{~m}, 2 \mathrm{H}) ;{ }^{13} \mathrm{C}$ NMR (75 MHz, DMSO-d6) $\delta 169.3$, $152.6,151.5,150.0,139.2,129.4,126.5,126.3,126.2$, 124.7, 123.0, 115.8, 114.1 , 79.2, 56.5, 53.2, 33.9, 29.7, 25.6, 22.4, 18.3; HRMS (ESI+) calc'd for $\mathrm{C}_{21} \mathrm{H}_{21} \mathrm{ClNO}_{3}{ }^{+}[\mathrm{M}+\mathrm{H}]^{+} 370.1210$ found 370.1204 .

\section{Synthesis of triarylmethane S10}

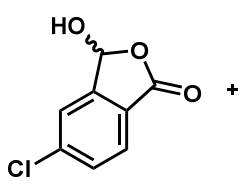

S05

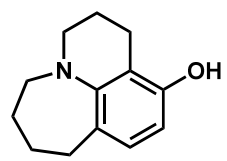

7

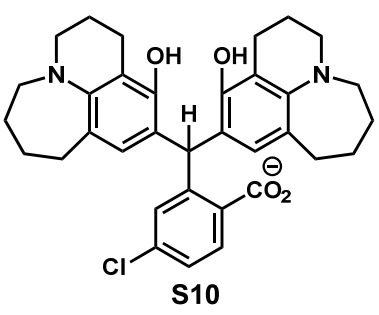

S10

To a $20 \mathrm{~mL}$ microwave vial flask equipped with stir bar was added 4chlorophthalaldehydic acid (47 mg, $0.25 \mathrm{mmol}$ ) and $7^{1}$ (155 mg, $\left.764 \mu \mathrm{mol}\right)$, followed by TFE (10 $\mathrm{mL}$ ). The resulting stirred solution was heated in microwave to $75^{\circ} \mathrm{C}$ under argon for 20 hours, at which point HPLC analysis indicated consumption of the starting material. Grey precipitate was filtered out (33 mg grey solid) and mother liquid was collected and solvent was removed in vacuo. 
Crude residue was purified by silica gel chromatography $(0 \rightarrow 30 \% \mathrm{MeOH} / \mathrm{DCM}$ to provide 60 $\mathrm{mg}$ of $\mathbf{S 1 0}$ as grey solid. Total yield (precipitate and after silica column purification) was $93 \mathrm{mg}$ (98\%) of grey solid. ${ }^{1} \mathrm{H}$ NMR (300 MHz, DMSO- $\left.d_{6}\right) \delta 12.82$ (br. s, $1 \mathrm{H}$ ), 7.81 (br. s, $2 \mathrm{H}$ ), 7.68 (d, $\mathrm{J}=8.3 \mathrm{~Hz}, 1 \mathrm{H}), 7.31(\mathrm{dd}, \mathrm{J}=8.3,2.0 \mathrm{~Hz}, 1 \mathrm{H}), 6.92(\mathrm{~d}, \mathrm{~J}=2.1 \mathrm{~Hz}, 1 \mathrm{H}), 6.83(\mathrm{~s}, 1 \mathrm{H}), 6.18(\mathrm{~s}, 2 \mathrm{H})$, $3.04-2.98(\mathrm{~m},, 4 \mathrm{H}), 2.89-2.80(\mathrm{~m}, 4 \mathrm{H}), 2.48-239$ (m, 8H, overlap with DMSO- $\left.d_{5}\right) 1.72-1.59$ $(\mathrm{m}, 8 \mathrm{H}), 1.45-1.26(\mathrm{~m}, 4 \mathrm{H}) ;{ }^{13} \mathrm{C}$ NMR $(75 \mathrm{MHz}$, DMSO-d 6$) \delta$ 168.8, 150.6, 147.3, 147.0, 134.8, 131.6, 130.0, 127.4, 125.6, 122.5, 114.8, 56.9, 53.7, 48.6, 34.6, 30.5, 26.3, 22.7, 18.2; HRMS (ESI+) calc'd for $\mathrm{C}_{34} \mathrm{H}_{38} \mathrm{ClN}_{2} \mathrm{O}_{4}{ }^{+}[\mathrm{M}+\mathrm{H}]^{+} 573.2520$ found 573.2490.

\section{Synthesis of triarylmethane S11}

Unoptimized reaction (failed attempt to synthesize dye 9)

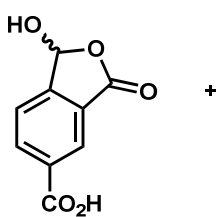

S04

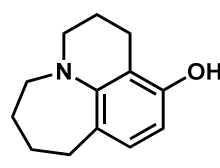

7

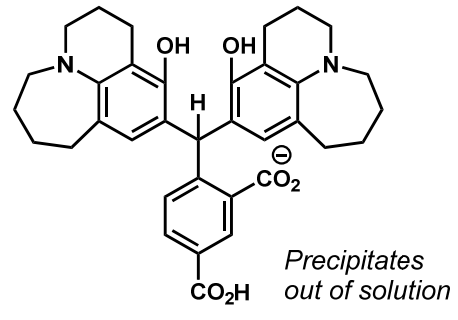

S11

To a $50 \mathrm{~mL}$ round bottom flask, equipped with stir bar was added 4chlorophthalaldehydic acid (120 mg, $0.620 \mathrm{mmol})$ and $7^{1}(314 \mathrm{mg}, 1.55 \mathrm{mmol})$, followed by TFE $(10 \mathrm{~mL})$. Oxygen was allowed to bubble through the solution for 5 minutes. The resulting clear yellow solution was gently warmed up under oxygen atmosphere to $80^{\circ} \mathrm{C}$. The resulting solution was stirred vigorously for 20 hours at which point deep red solution had a lot of fine white precipitate. Precipitate was filtered out and dried under vacuum to provide $250 \mathrm{mg}$ (72\% yield) of S11 as bluish white solid. ${ }^{1} \mathrm{H}$ NMR (300 MHz, DMSO- $\left.d_{6}\right) \delta 13.00$ (br.s, $\left.2 \mathrm{H}\right), 8.23$ (d, J = 2.0, $1 \mathrm{H}$ ), 7.94 (dd, $J=8.1,2.0 \mathrm{~Hz}, 1 \mathrm{H}), 7.74$ (br. s, 2H), 7.14 (d, $J=8.2 \mathrm{~Hz}, 1 \mathrm{H}), 6.86$ (s, 1H), 6.20 (s, 2H), $3.12-2.95(\mathrm{~m}, 4 \mathrm{H}), 2.93-2.78(\mathrm{~m}, 4 \mathrm{H}), 2.45-2.32(\mathrm{~m}, 4 \mathrm{H}), 1.75-1.56(\mathrm{~m}, 8 \mathrm{H}), 1.56-1.09$ $(\mathrm{m}, 4 \mathrm{H}) ;{ }^{13} \mathrm{C}$ NMR $\left(75 \mathrm{MHz}, \mathrm{DMSO}-d_{6}\right) \delta 168.9,166.8,150.6,150.2,147.0,132.1,131.13$, 131.07, 130.7, 128.0, 127.6, 125.6, 122.6, 114.7, 56.9, 53.7, 34.6, 30.5, 26.2, 22.7, 18.0; HRMS $(\mathrm{ESI}+)$ calc'd for $\mathrm{C}_{35} \mathrm{H}_{39} \mathrm{~N}_{2} \mathrm{O}_{6}{ }^{+}[\mathrm{M}+\mathrm{H}]^{+} 583.2808$ found 583.2792 


\section{Synthesis of triarylmethane S12}

Unoptimized reaction (failed attempt to synthesize dye 21)
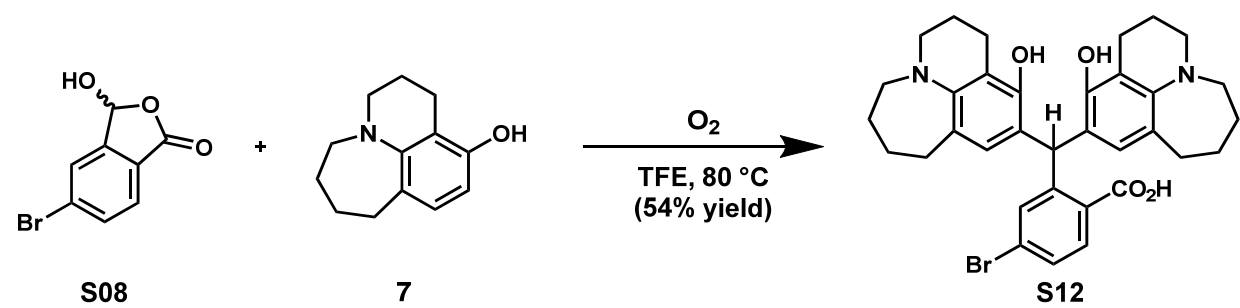

A $50 \mathrm{~mL}$ round-bottom flask equipped with stir bar was charged with 4bromophthalaldehydic acid ${ }^{2}(\mathbf{S 0 8})(65.0 \mathrm{mg}, 284 \mu \mathrm{mol})$, phenol $7^{1}(141 \mathrm{mg}, 596 \mu \mathrm{mol})$ and trifluoroethanol $(20 \mathrm{~mL})$. Oxygen was allowed to bubble through the solution for 5 minutes. The resulting clear yellow solution was gently warmed up under oxygen atmosphere to $80{ }^{\circ} \mathrm{C}$. The resulting solution was stirred vigorously for 22 hours. Fine white precipitate was filtered out from the deep red solution to provide $90 \mathrm{mg}$ (53\% yield) of $\mathbf{S 1 2}$ as bluish solid. ${ }^{1} \mathrm{H}$ NMR (300 MHz, DMSO-d6) $\delta 12.75$ (br. s, 1H), 7.72 (br. s, 2H), 7.62 (d, J = 8.3 Hz, 1H), 7.44 (dd, J = 8.3, 2.1 Hz, $1 \mathrm{H}), 7.05(\mathrm{~d}, \mathrm{~J}=2.1 \mathrm{~Hz}, 1 \mathrm{H}), 6.81(\mathrm{~s}, 1 \mathrm{H}), 6.16(\mathrm{~s}, 2 \mathrm{H}), 3.05-2.96(\mathrm{~m}, 4 \mathrm{H}), 2.90-2.81(\mathrm{~m}, 4 \mathrm{H})$, $2.48-2.37\left(\mathrm{~m}, 8 \mathrm{H}\right.$, overlap with DMSO- $\left.d_{5}\right), 1.72-1.59(\mathrm{~m}, 8 \mathrm{H}), 1.46-1.28(\mathrm{~m}, 4 \mathrm{H}) ;{ }^{13} \mathrm{C} \mathrm{NMR}$ (75 MHz, DMSO-d $d_{6} \delta 168.7,150.5,147.8,146.9,132.9,131.8,131.2,128.6,127.5,125.6,124.2$, 122.5, 114.8, 56.9, 53.7, 34.6, 30.4, 26.2, 22.7, 18.0; HRMS (ESI+) calc'd for $\mathrm{C}_{34} \mathrm{H}_{38} \mathrm{BrN}_{2} \mathrm{O}_{4}{ }^{+}$ $[\mathrm{M}+\mathrm{H}]^{+} 617.2015$ found 617.2010 .

\section{Solvent Screen: General Procedure for Solvent Screen (Table 1).}

Entries $1-8$ open to air

Entries $9-10$ under $\mathrm{O}_{2}$ balloon

A $50 \mathrm{~mL}$ round bottom flask, equipped with stir bar, was charged with 4carboxyphthalaldehydic acid $(29.1 \mathrm{mg}, 150 \mu \mathrm{mol})$ and phenol $7^{1}(76.1 \mathrm{mg}, 375 \mu \mathrm{mol})$. An appropriate solvent was added $(5 \mathrm{~mL})$ and the resulting solution was stirred $80^{\circ} \mathrm{C}\left(60^{\circ} \mathrm{C}\right.$ for HFIP $)$ open to air for 20 hours. The solvent was removed in vacuo and known a amount of mesitylene 
(internal standard) was added $(\sim 100 \mu \mathrm{mol})$ in DMSO- $d_{6}(5 \mathrm{~mL}) .{ }^{3}$ When everything dissolved, 0.6 $\mathrm{mL}$ of the solution was transferred to an NMR tube and the yields of 10, 14 and $\mathbf{1 5}$ were derived from the relative ratio to mesitylene peak. ${ }^{4}$

\section{References:}

(1) Kirkland, T. A.; McDougall, M. G.; Dwight, S. WO 2013078244, 2013.

(2) Purchased form Matrix Scientific

(3) Excact amount of mesitylene was measured by weight of material added each time

(4) A 15 second delay time (D1) was employed for all ${ }^{1} \mathrm{H}$ NMR measurements. 


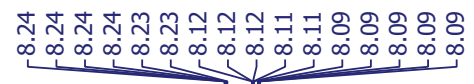

$300 \mathrm{MHz}{ }^{1} \mathrm{H}$ NMR Spectrum of $\mathbf{S 0 2}$ in DMSO- $d_{6}$

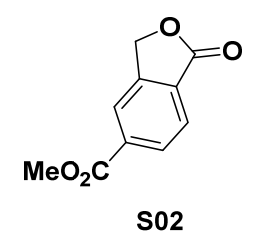

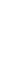
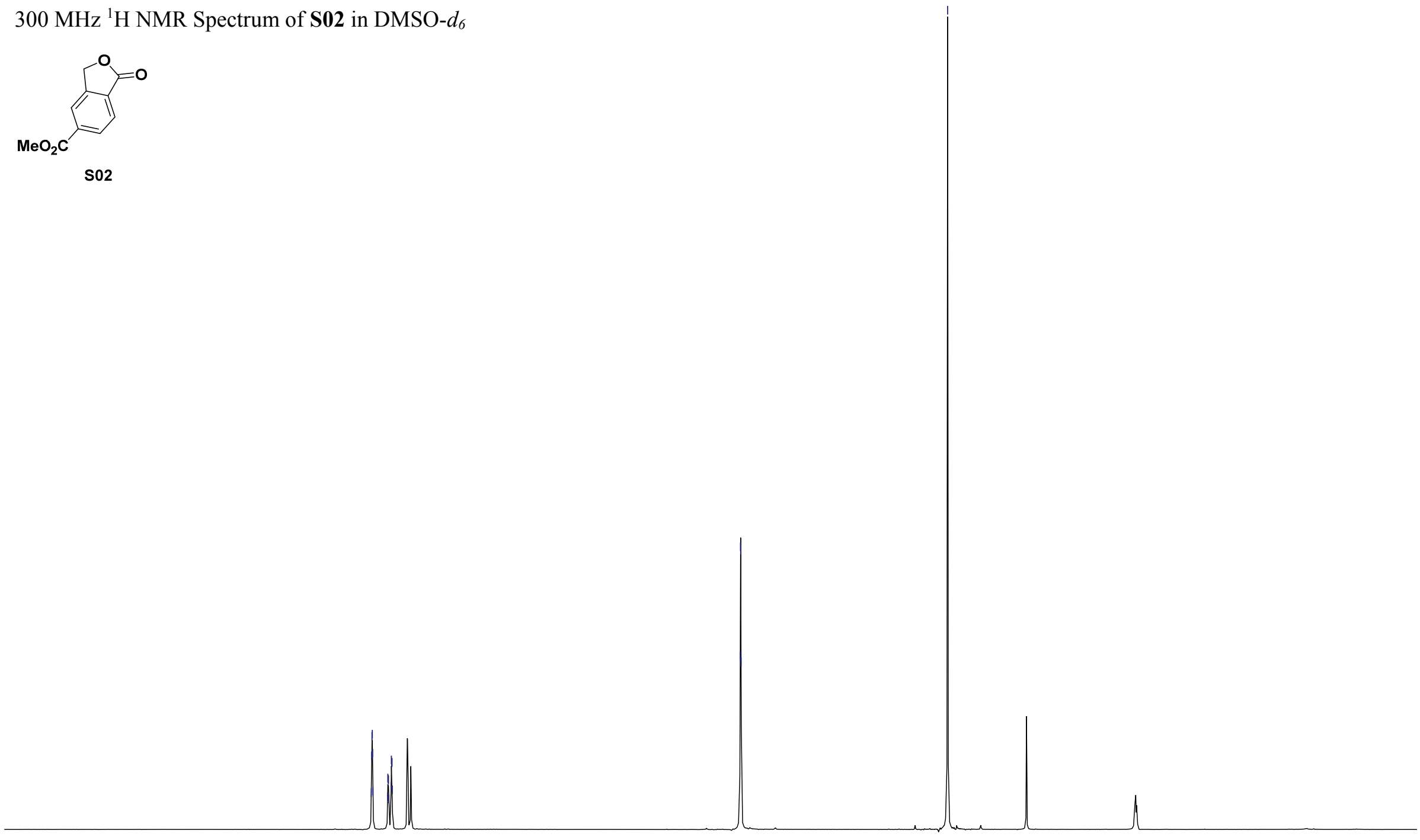

\begin{tabular}{|c|c|c|c|c|c|c|c|c|c|c|c|c|c|c|c|c|c|c|c|c|c|}
\hline & & & & & & 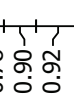 & & & & & † & & & † & & & & & & & \\
\hline .0 & 10.5 & 10.0 & 9.5 & 9.0 & 8.5 & 8.0 & 7.5 & 7.0 & 6.5 & 6.0 & 5.5 & 5.0 & 4.5 & 4.0 & 3.5 & 3.0 & 2.5 & 2.0 & 1.5 & 1.0 & 0.5 \\
\hline
\end{tabular}




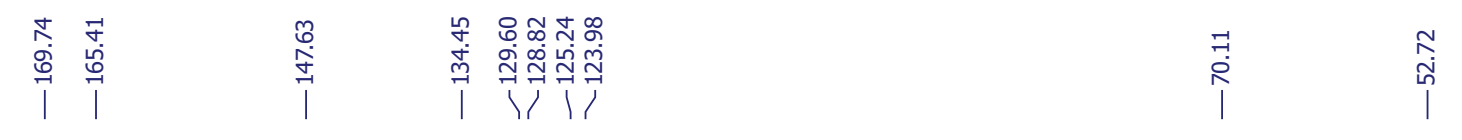

$75 \mathrm{MHz}{ }^{13} \mathrm{C}$ NMR Spectrum of S02 in DMSO- $d_{6}$
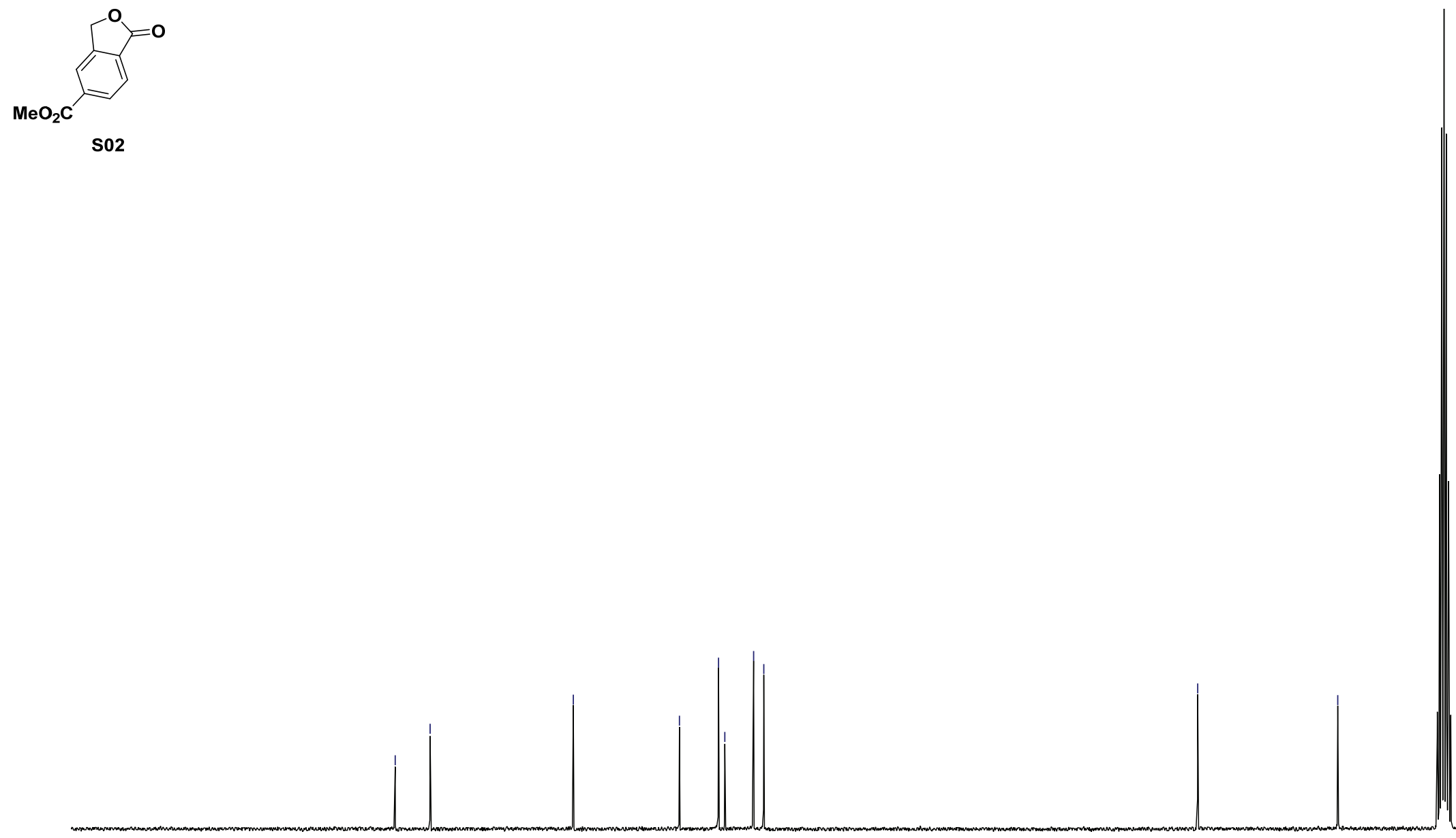

$10 \quad 200 \quad 190$

180

$170 \quad 160$

150

140

130

120

110

100

90

80

70

60

50 
$300 \mathbf{M H z}{ }^{1} \mathrm{H}$ NMR Spectrum of $\mathbf{S 0 3}$ in $\mathrm{CDCl}_{3}$

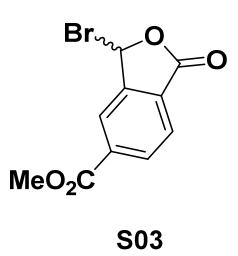

s03

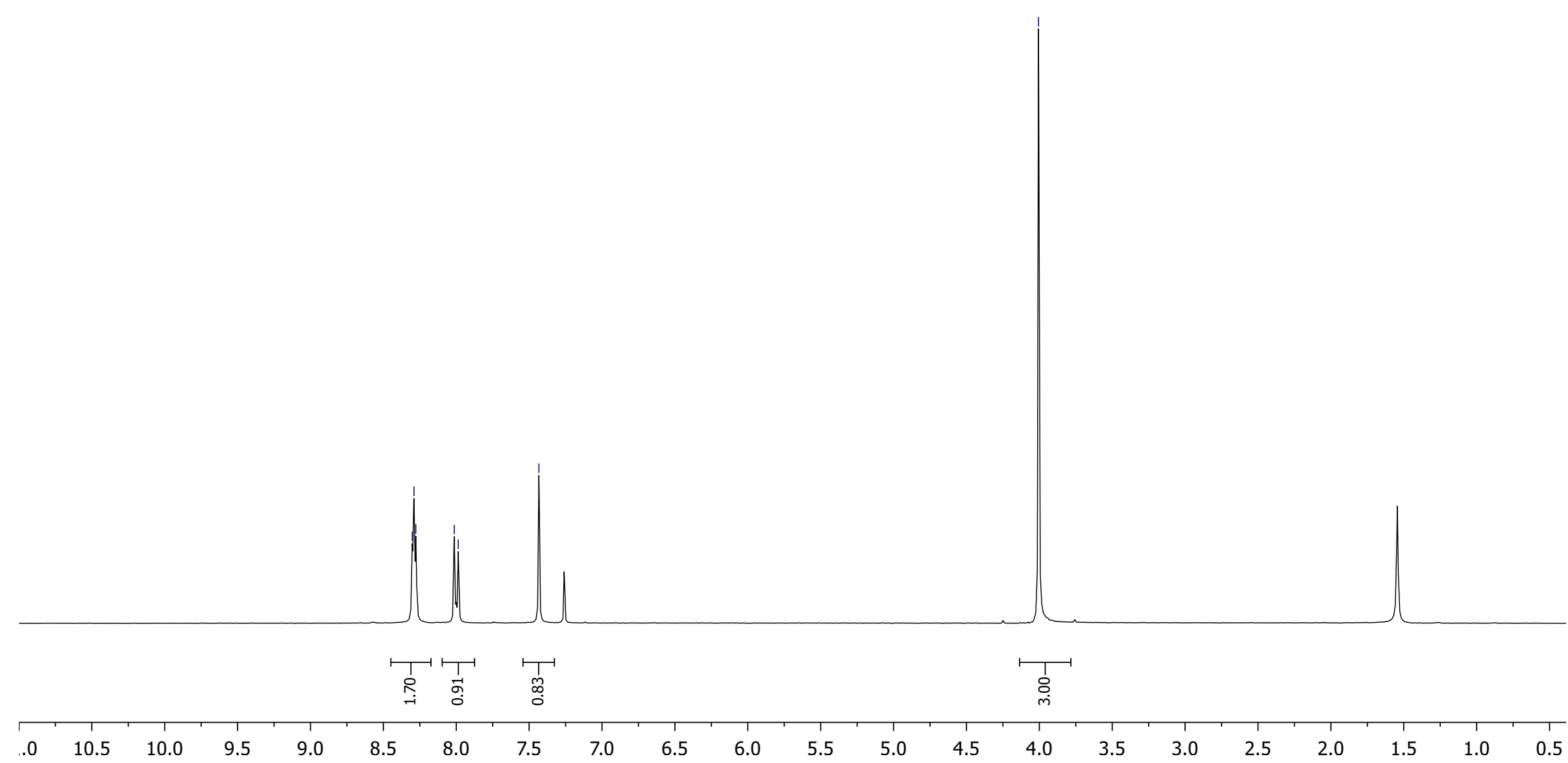




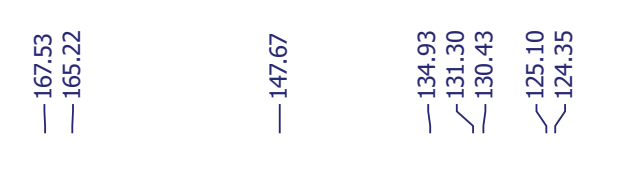

$75 \mathrm{MHz}{ }^{13} \mathrm{C}$ NMR Spectrum of $\mathbf{S 0 3}$ in DMSO- $d_{6}$
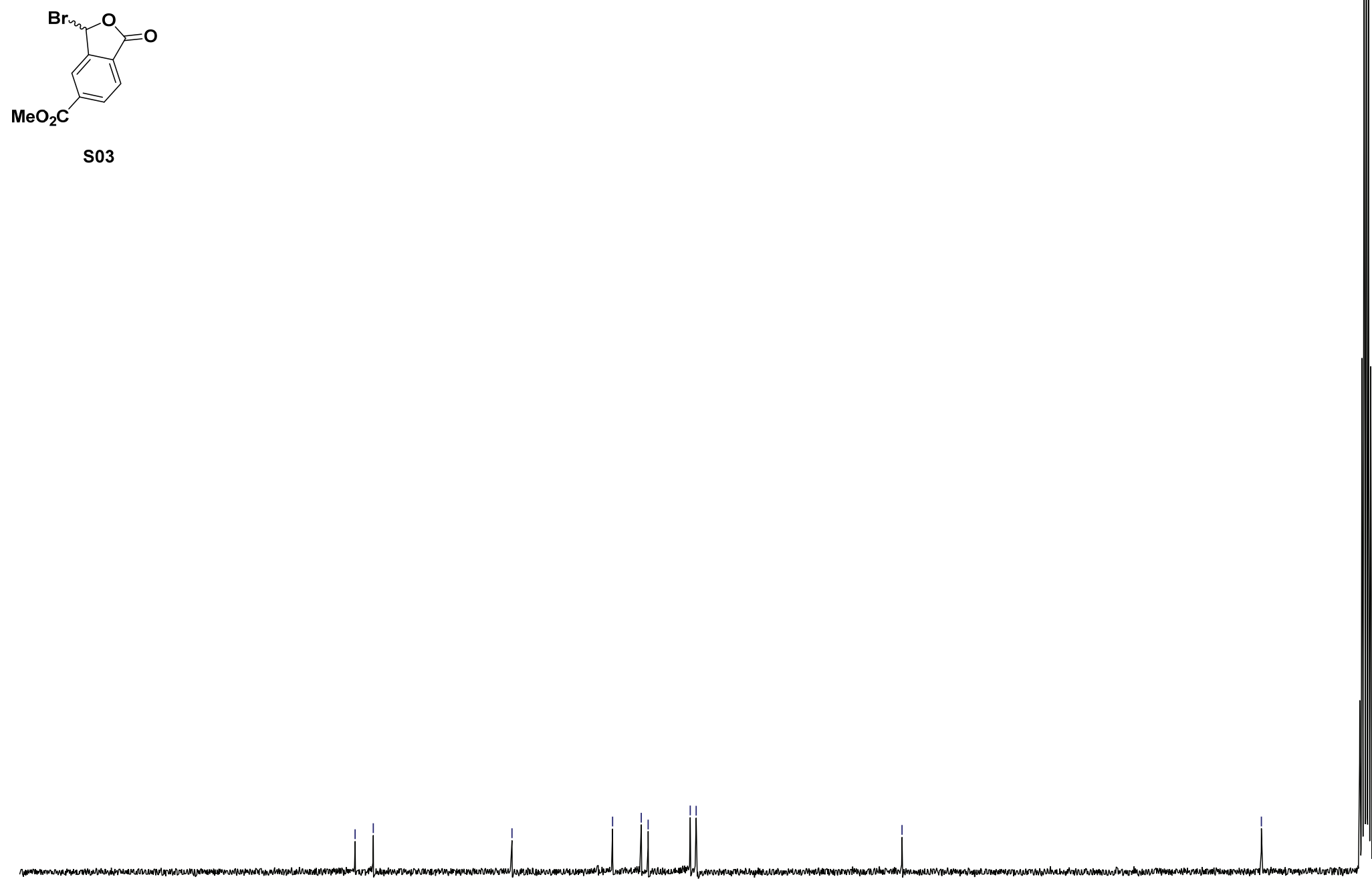


\section{|}

$300 \mathrm{MHz}{ }^{1} \mathrm{H}$ NMR Spectrum of S05 in DMSO- $d_{6}$

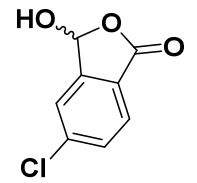

S05

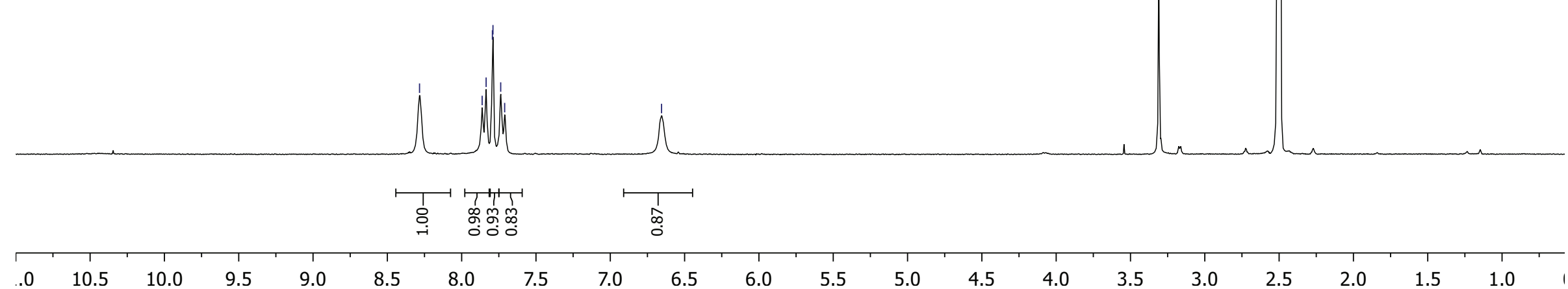




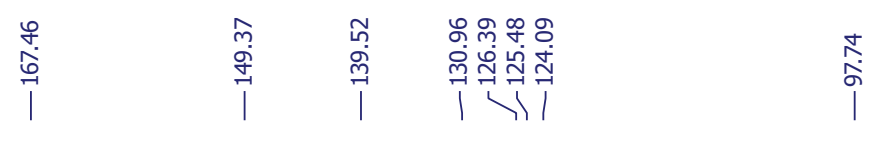

$75 \mathrm{MHz}{ }^{13} \mathrm{C}$ NMR Spectrum of $\mathbf{S 0 5}$ in DMSO- $d_{6}$

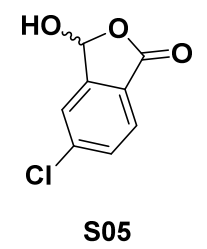

S05
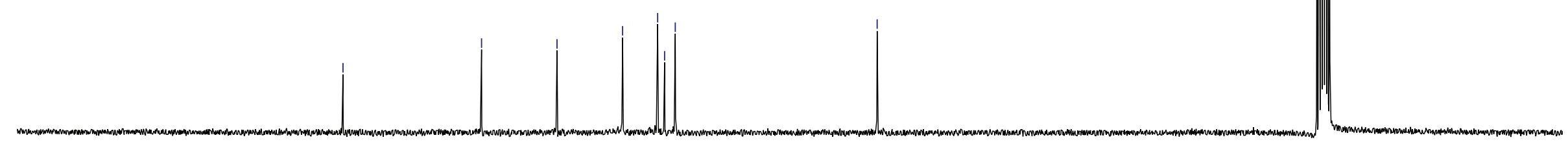

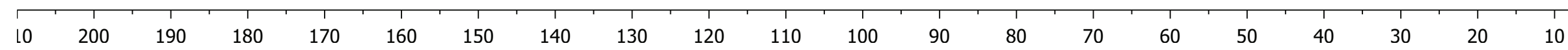


।

$300 \mathbf{M H z}{ }^{1} \mathrm{H}$ NMR Spectrum of $\mathbf{S 0 7}$ in DMSO- $d_{6}$

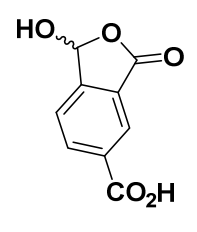

$\mathrm{S} 07$

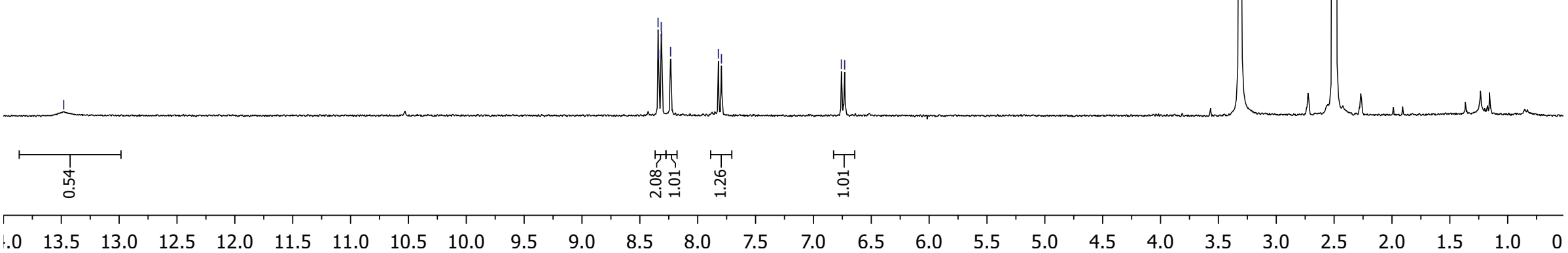




il

$75 \mathrm{MHz}{ }^{13} \mathrm{C}$ NMR Spectrum of $\mathbf{S 0 7}$ in DMSO- $d_{6}$

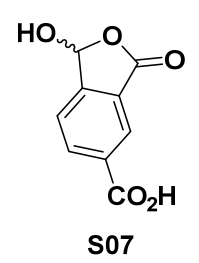

S07

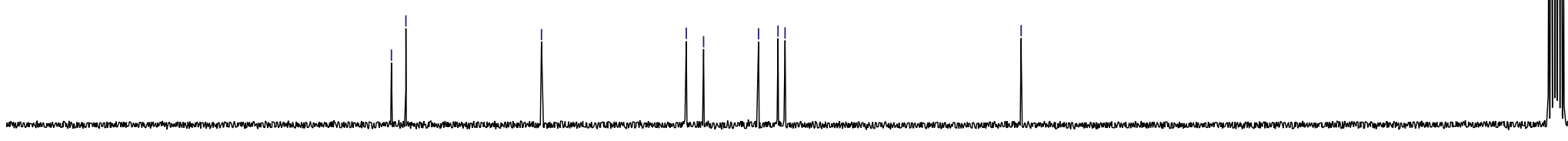

10

200

190

180

170

160

150

140

130

120

110

100

90

80

70

60

50

40 


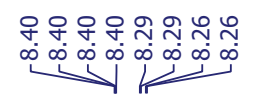

$300 \mathrm{MHz}{ }^{1} \mathrm{H}$ NMR Spectrum of 9 in DMSO- $d_{6}$

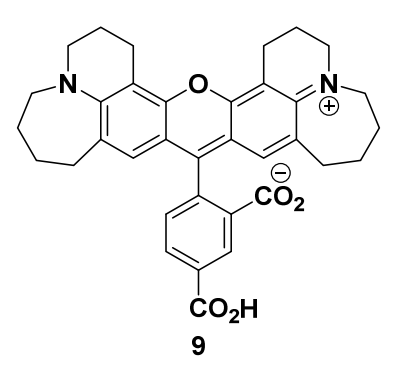

9

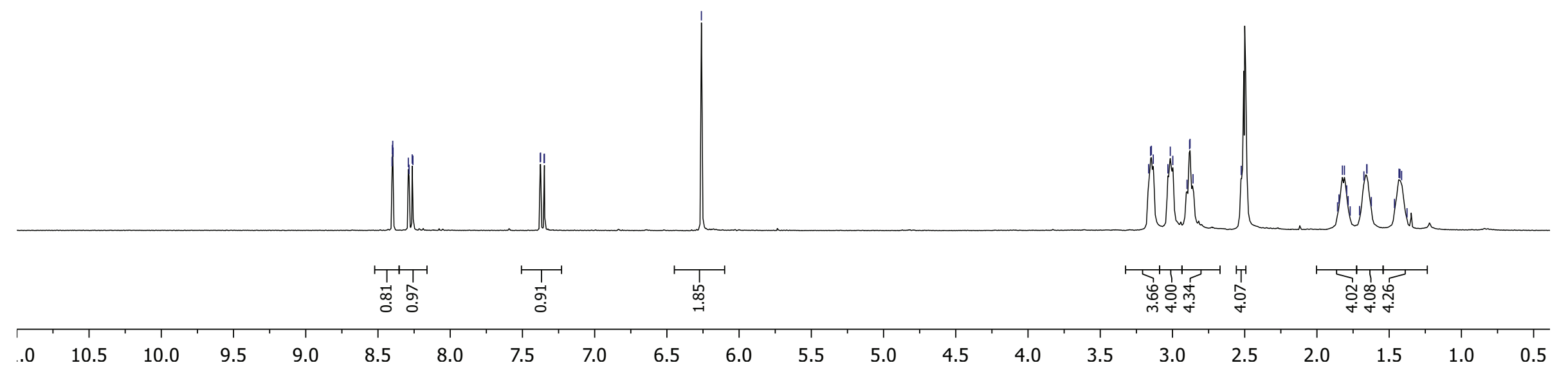


$75 \mathrm{MHz}{ }^{13} \mathrm{C}$ NMR Spectrum of 9 in DMSO- $d_{6}$

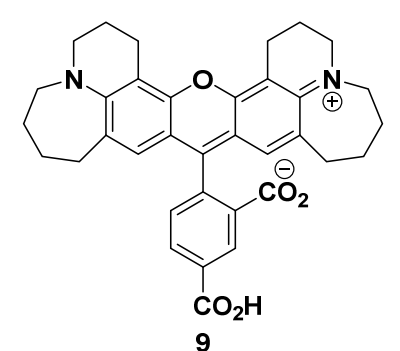

9
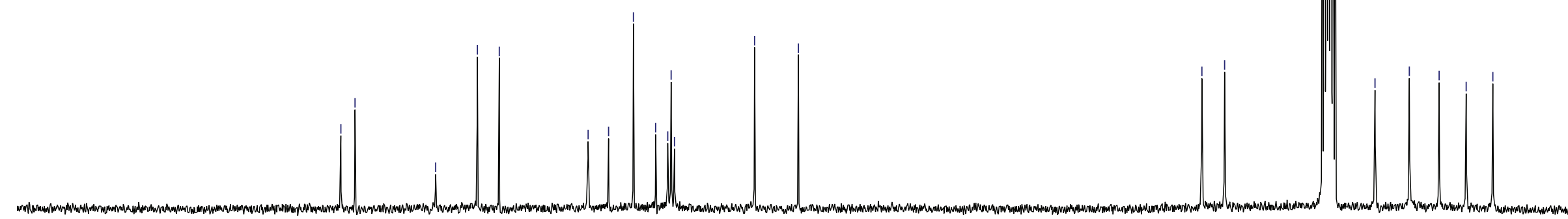

$$
10
$$

200

190

180

170

160

150

140

130

120

110

100

90

80

70

60

50

40

30

20 
$300 \mathrm{MHz}{ }^{1} \mathrm{H}$ NMR Spectrum of $\mathbf{1 0}$ in DMSO- $d_{6}$
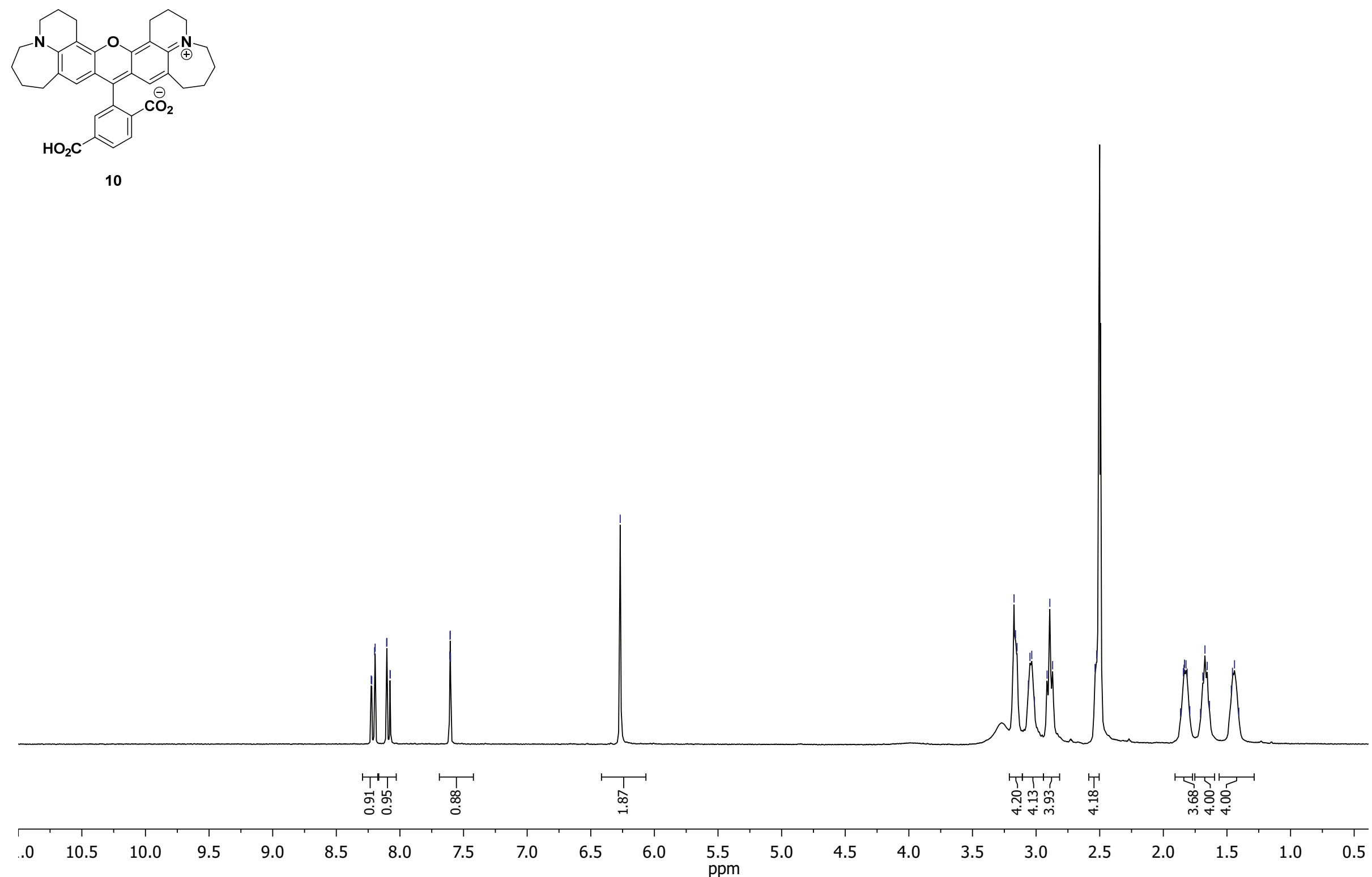


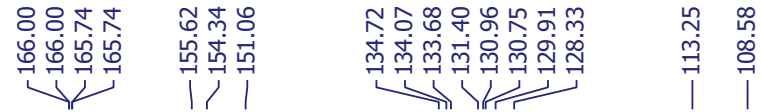

$75 \mathrm{MHz}{ }^{13} \mathrm{C}$ NMR Spectrum of $\mathbf{1 0}$ in DMSO- $d_{6}$
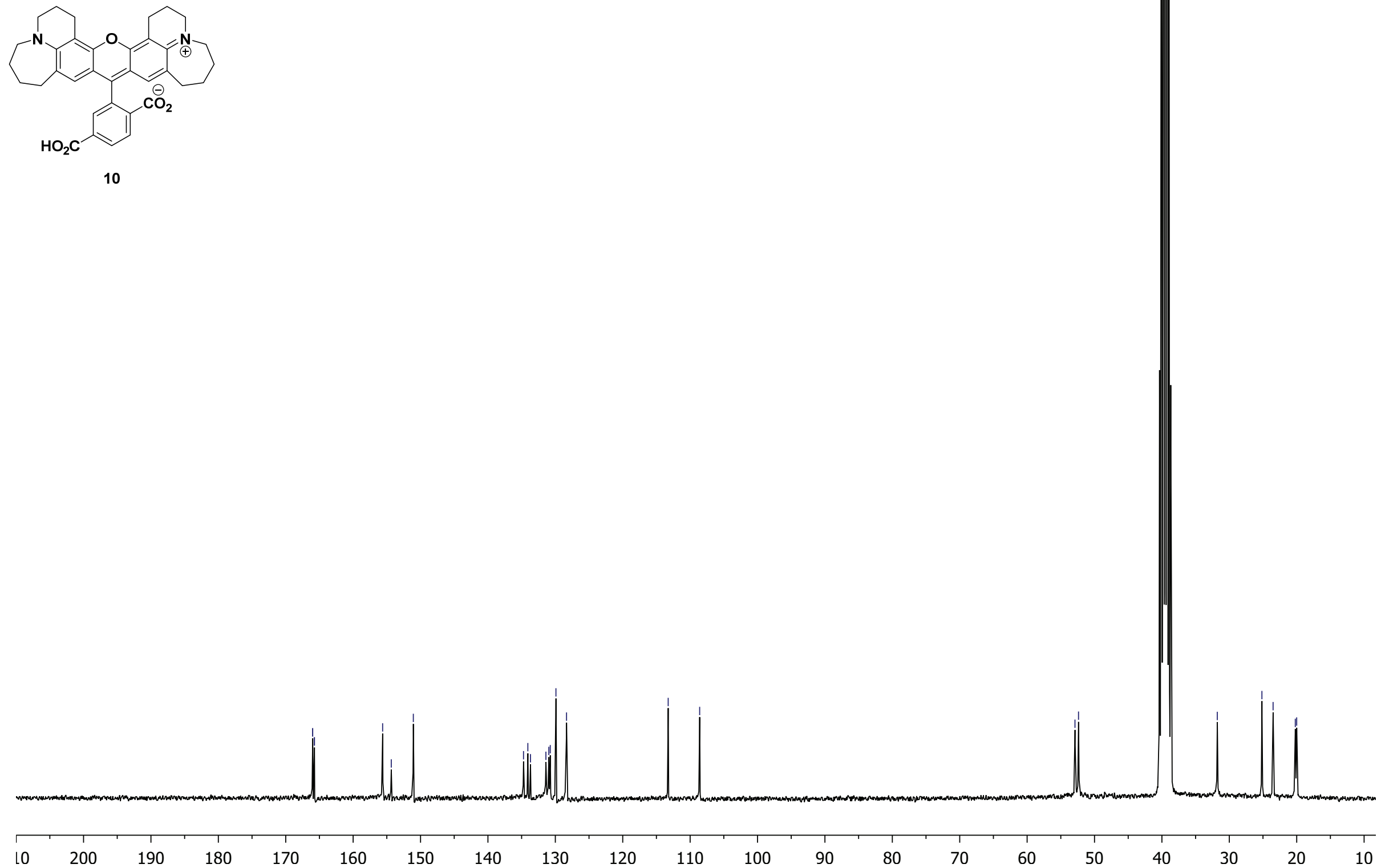
$300 \mathrm{MHz}{ }^{1} \mathrm{H}$ NMR Spectrum of $\mathbf{1 1}$ in DMSO- $d_{6}$
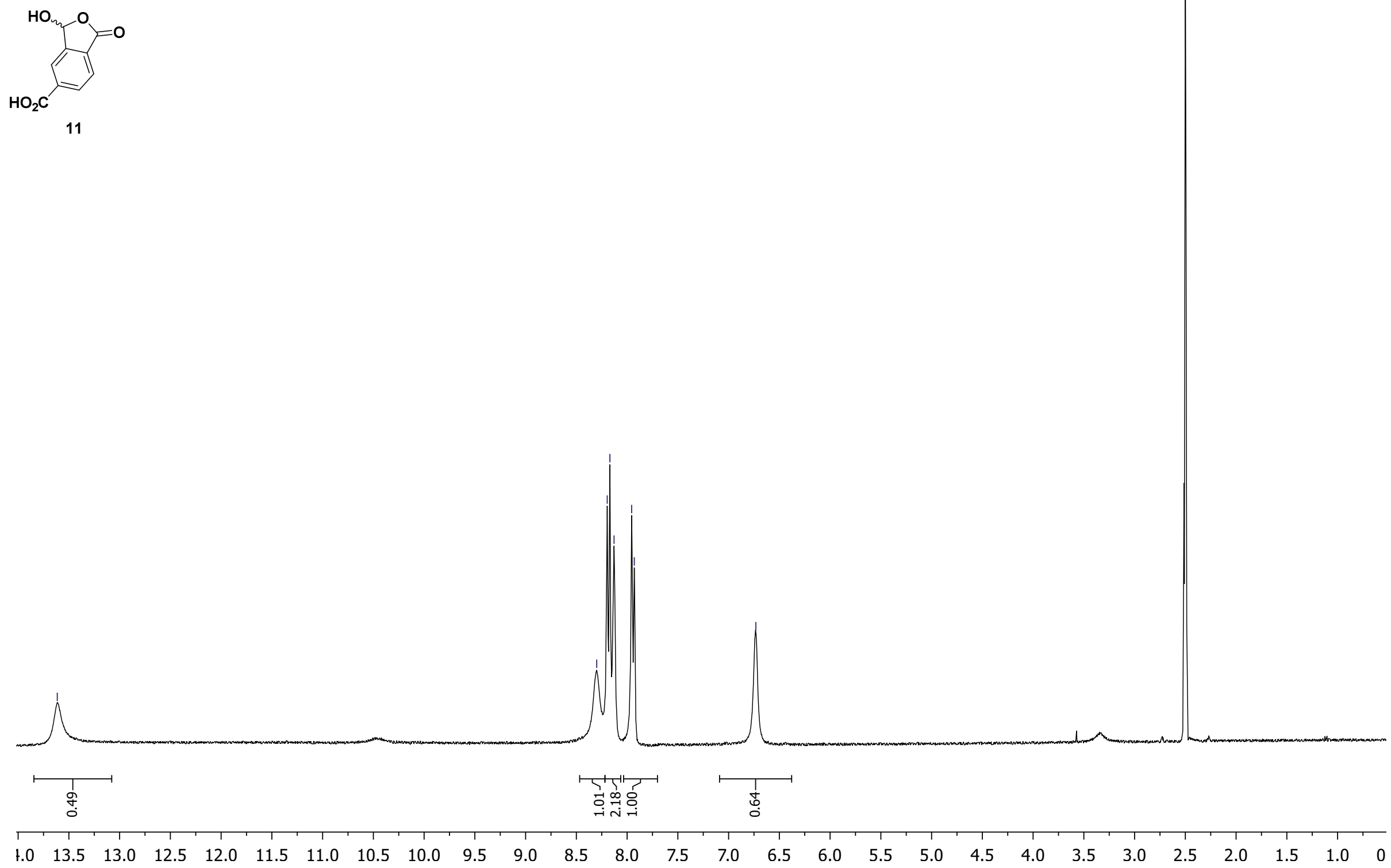


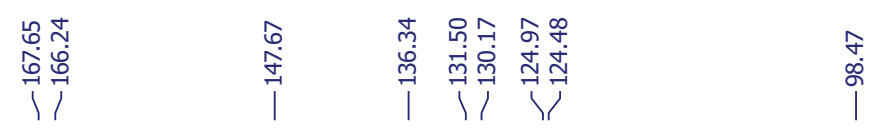

$75 \mathrm{MHz}{ }^{13} \mathrm{C}$ NMR Spectrum of 11 in DMSO- $d_{6}$
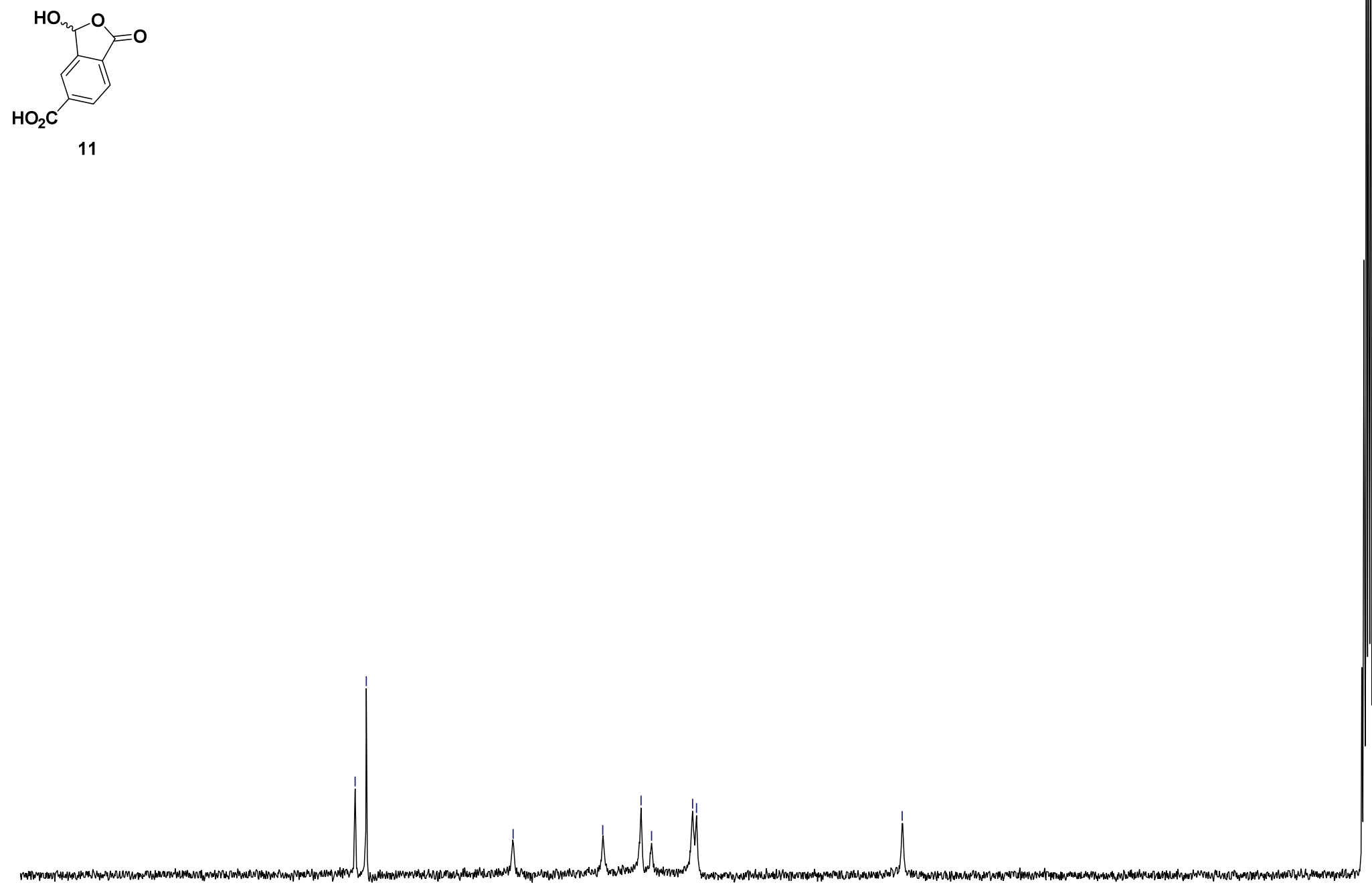
$300 \mathrm{MHz}{ }^{1} \mathrm{H}$ NMR Spectrum of $\mathbf{1 4}$ in DMSO- $d_{6}$
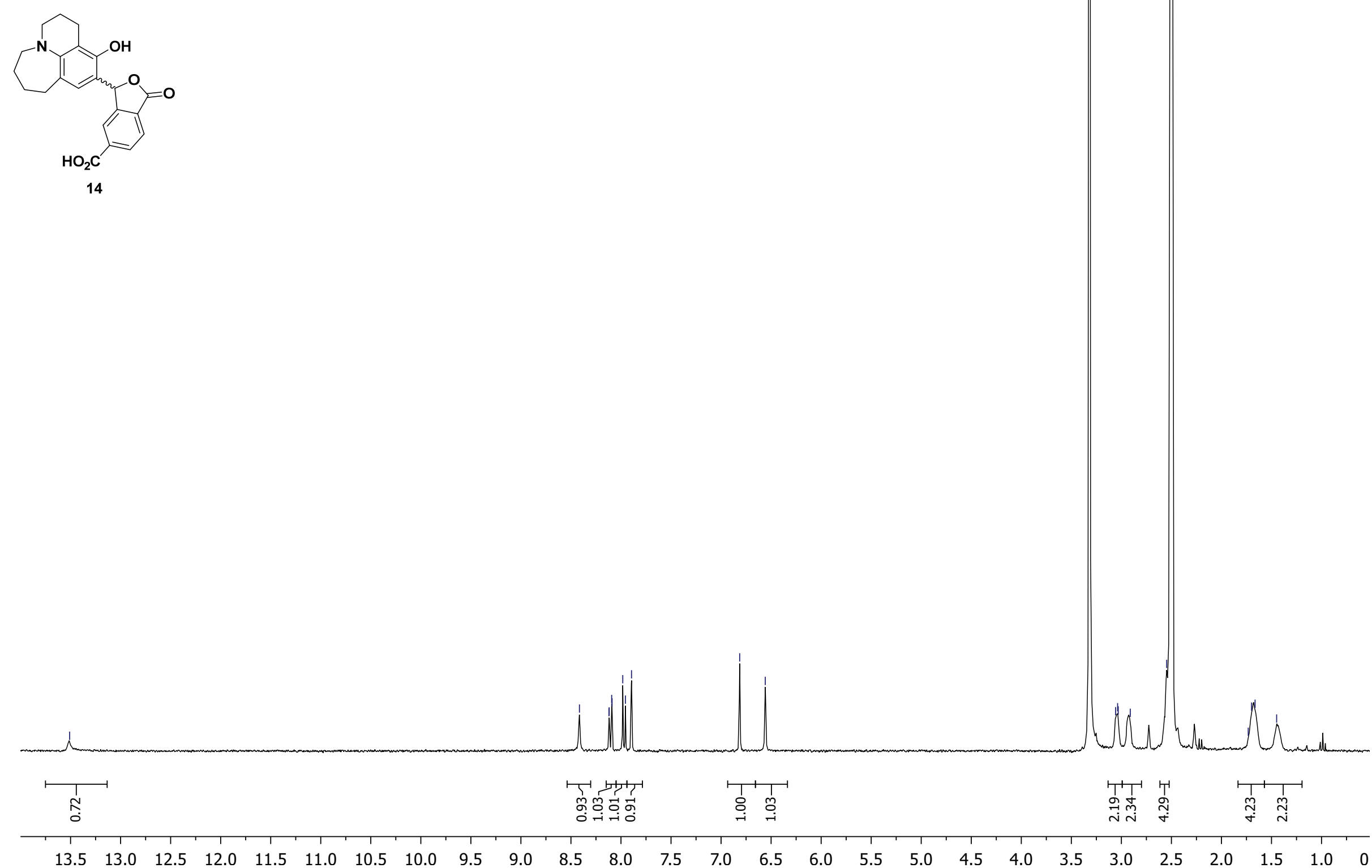


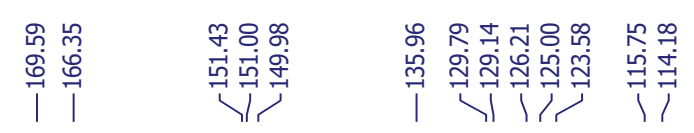

$75 \mathrm{MHz}{ }^{13} \mathrm{C}$ NMR Spectrum of $\mathbf{1 4}$ in DMSO- $d_{6}$

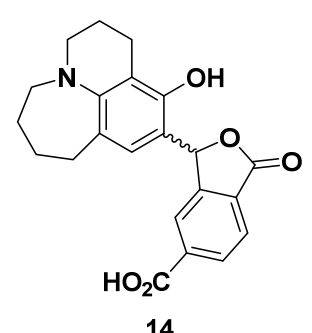

14

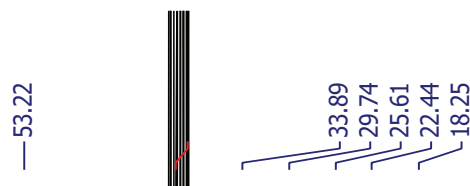

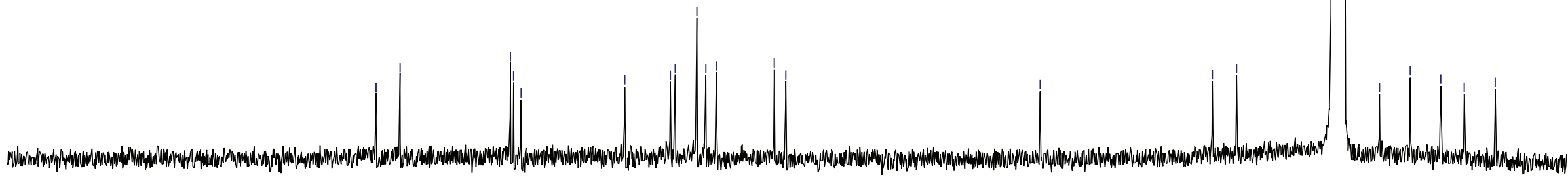

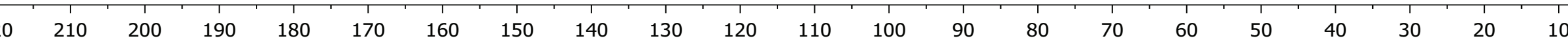




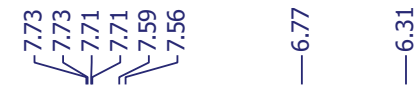

$300 \mathrm{MHz}{ }^{1} \mathrm{H}$ NMR Spectrum of $\mathbf{1 5}$ in DMSO- $d_{6}$
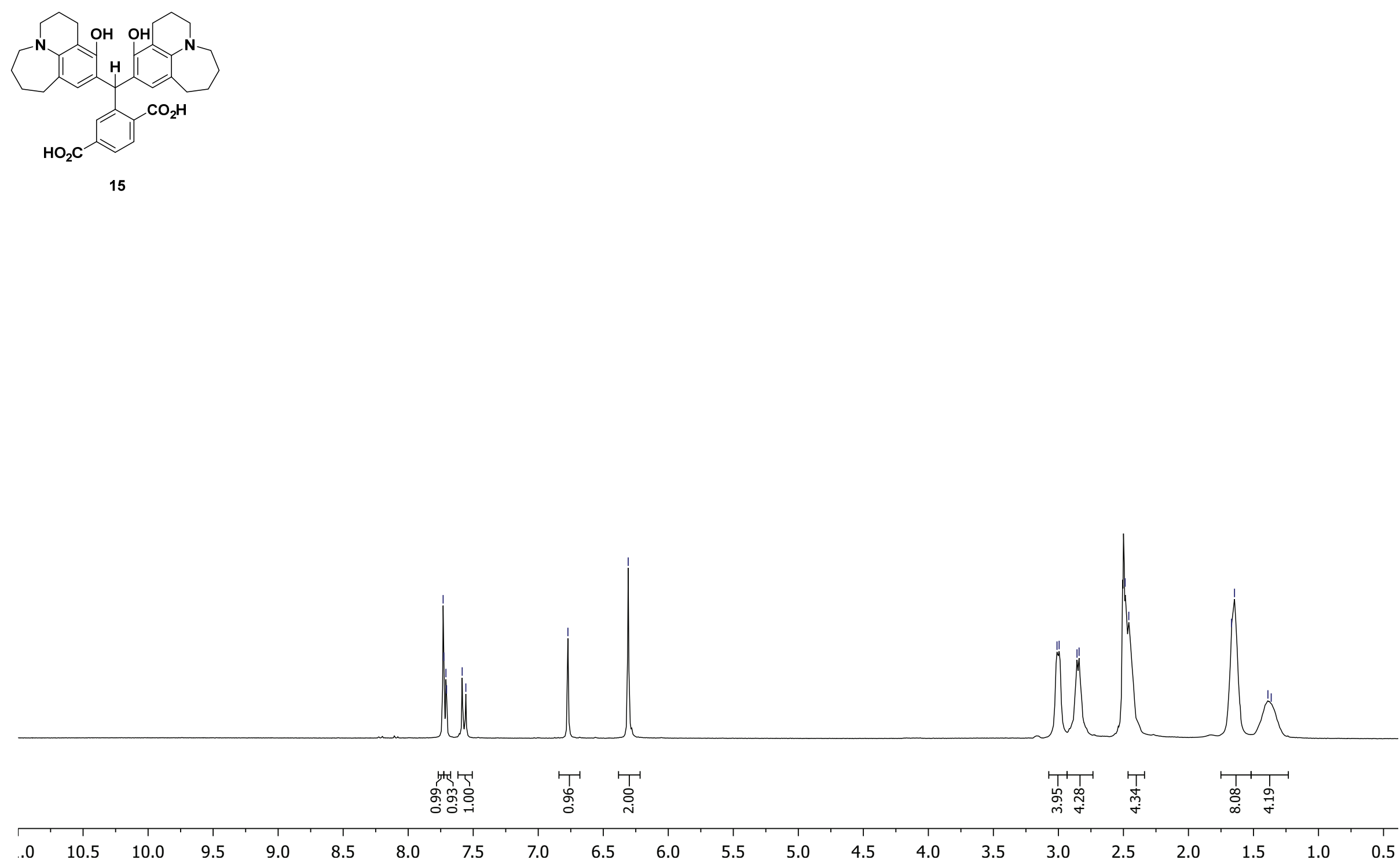


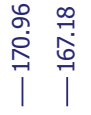

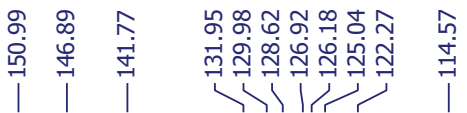

$75 \mathrm{MHz}{ }^{13} \mathrm{C}$ NMR Spectrum of 15 in DMSO- $d_{6}$
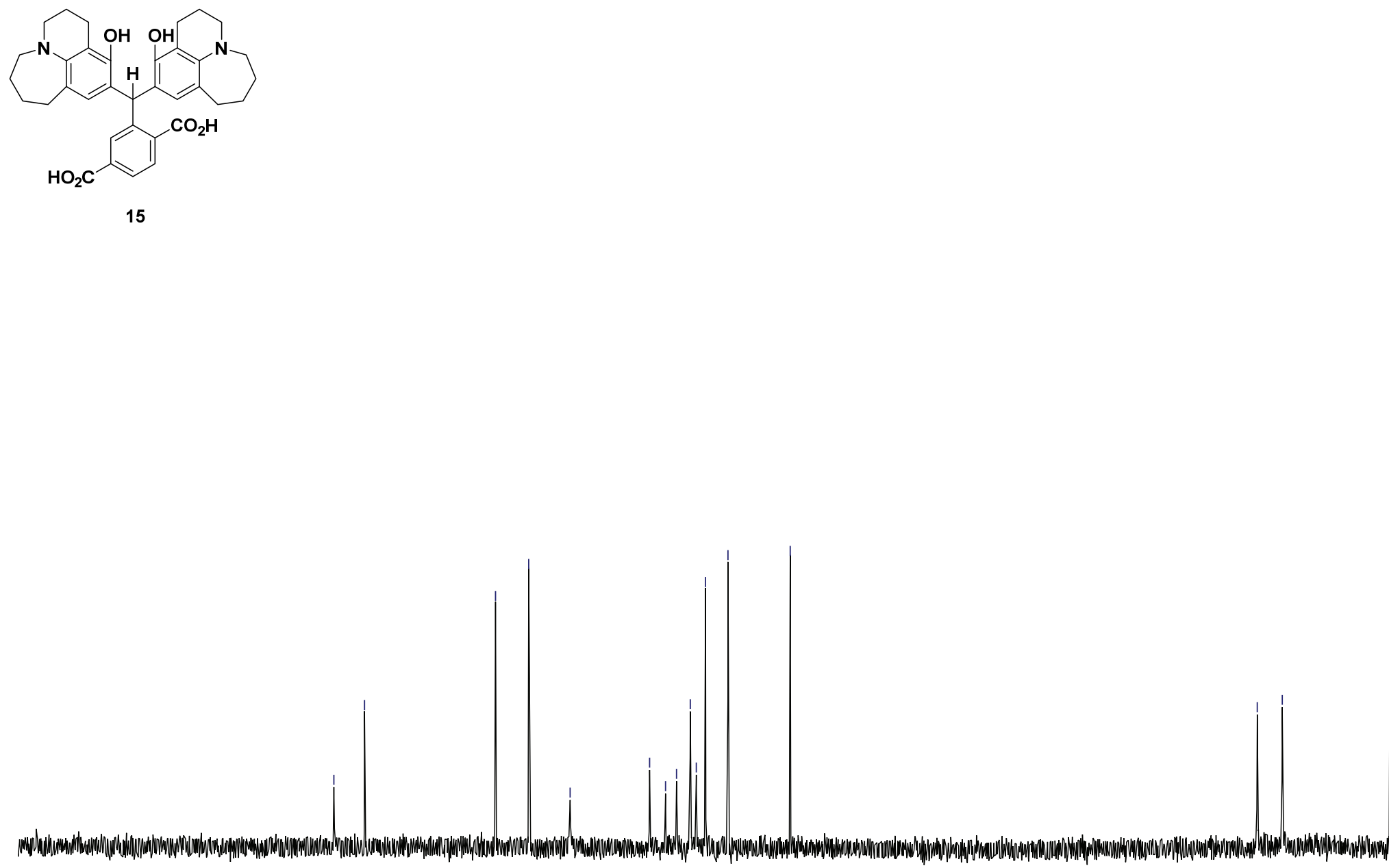


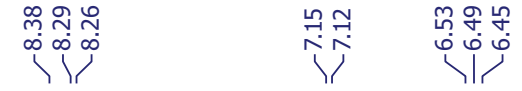

$300 \mathrm{MHz}{ }^{1} \mathrm{H}$ NMR Spectrum of 16 in DMSO- $\mathrm{d}_{6}$

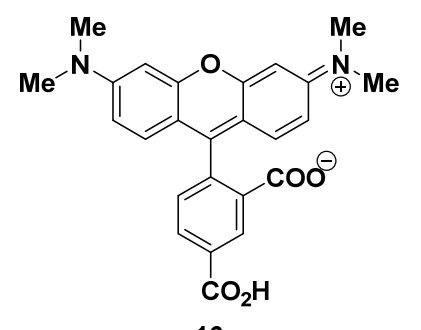

16

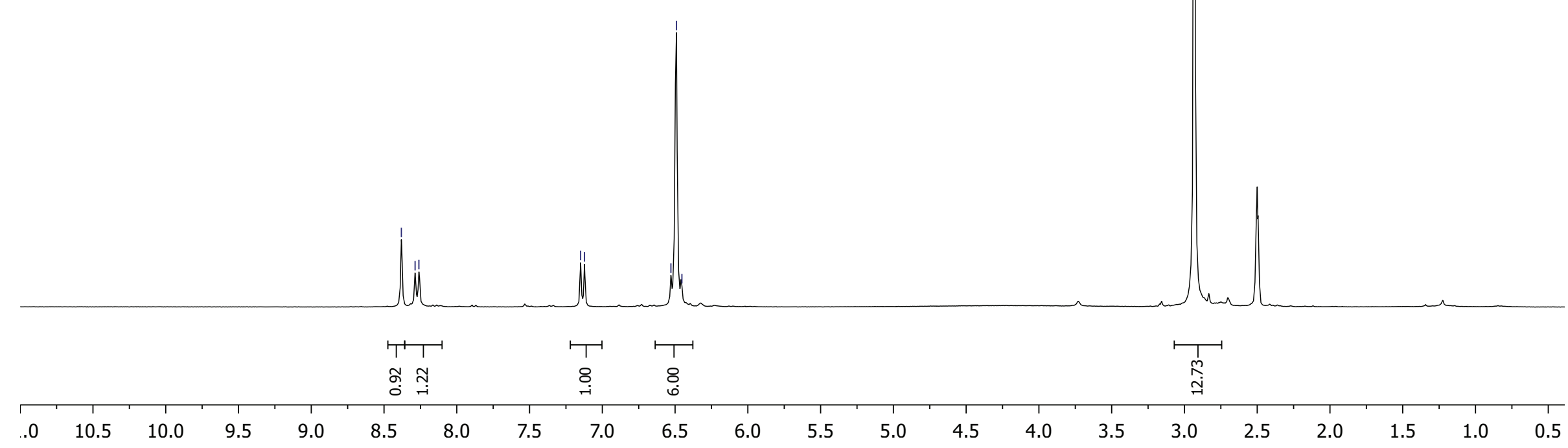




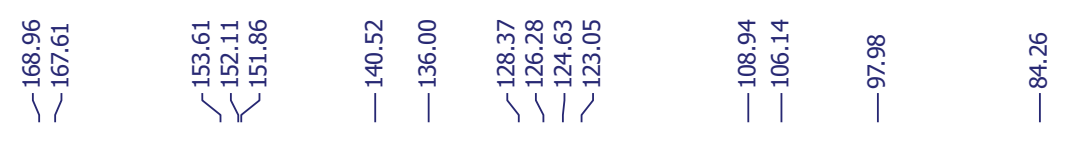

$75 \mathrm{MHz}{ }^{13} \mathrm{C}$ NMR Spectrum of $\mathbf{1 6}$ in DMSO-d 6

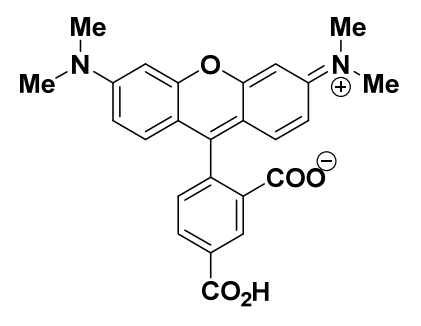

16
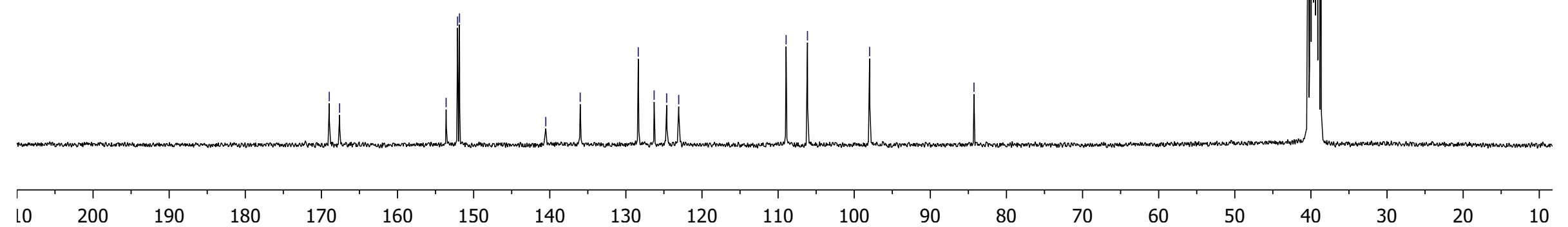
$300 \mathrm{MHz}{ }^{1} \mathrm{H}$ NMR Spectrum of $\mathbf{1 7}$ in $\mathrm{CD}_{3} \mathrm{OD}$
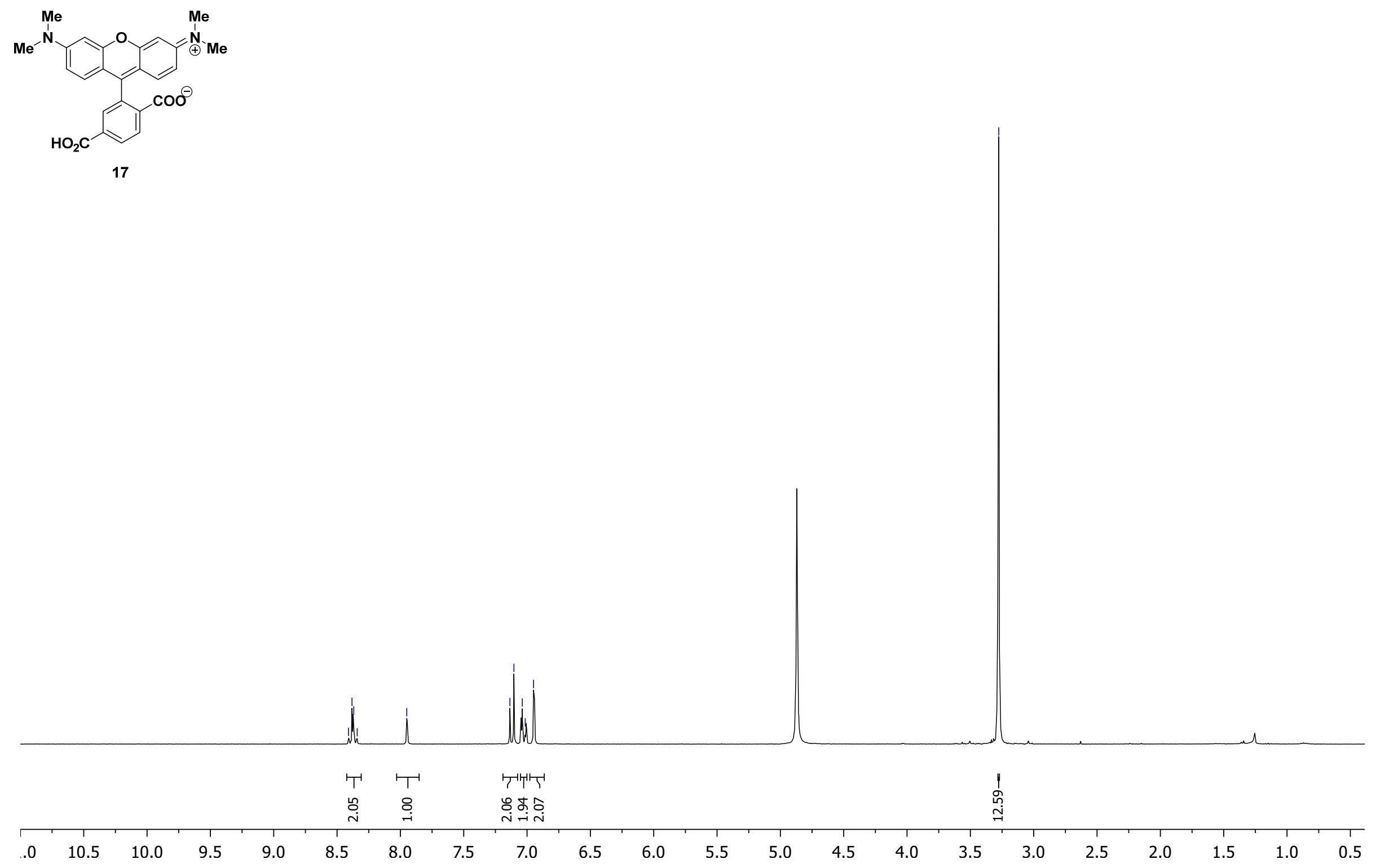


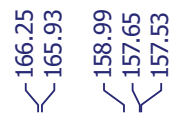

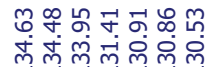 \\ 然堽 \\ 1) \\ i̊}

$75 \mathrm{MHz}{ }^{13} \mathrm{C}$ NMR Spectrum of $\mathbf{1 7}$ in $\mathrm{CD}_{3} \mathrm{OD}$
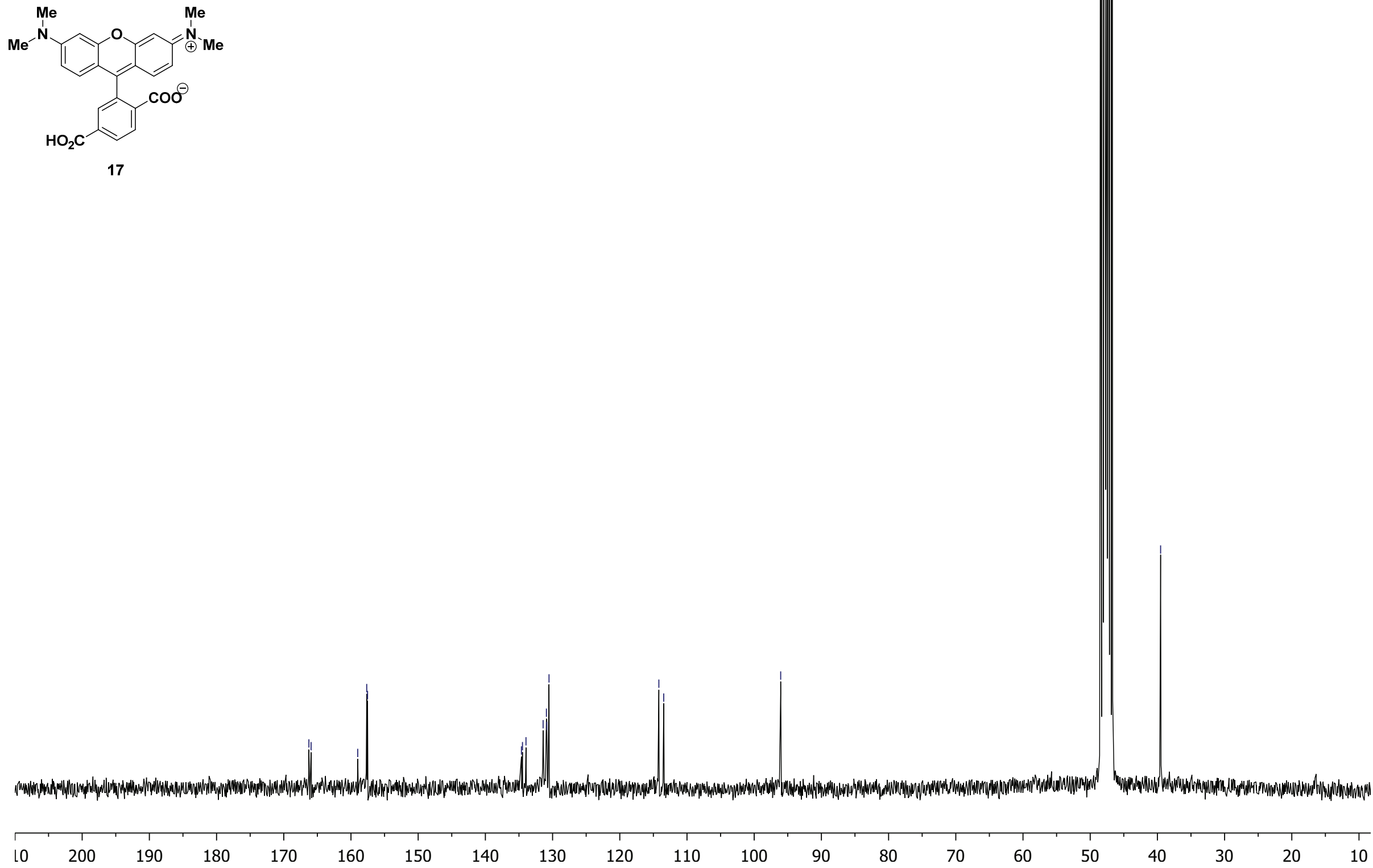
$300 \mathrm{MHz}{ }^{1} \mathrm{H}$ NMR Spectrum of $\mathbf{1 8}$ in DMSO- $d_{6}$

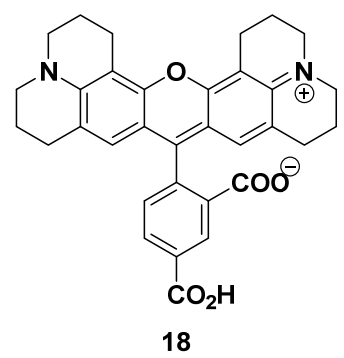

18

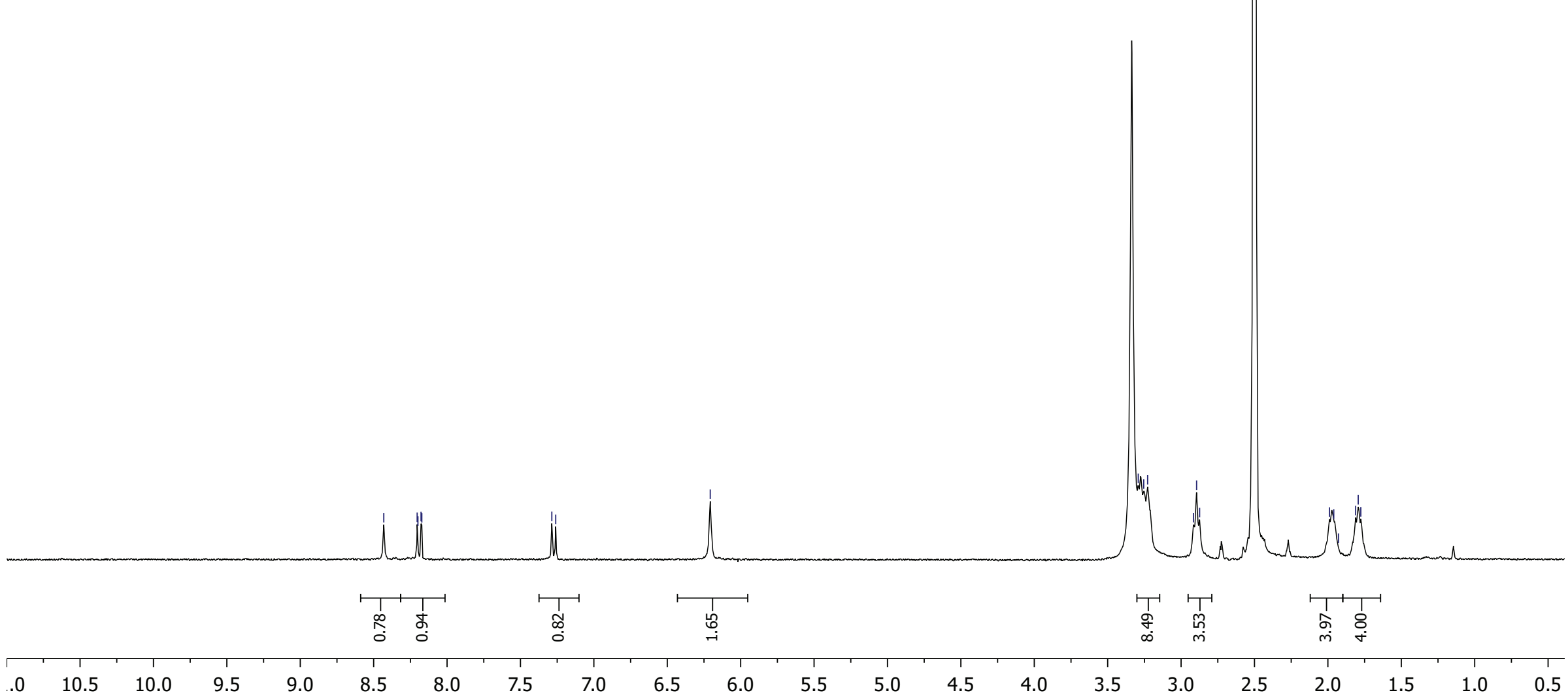




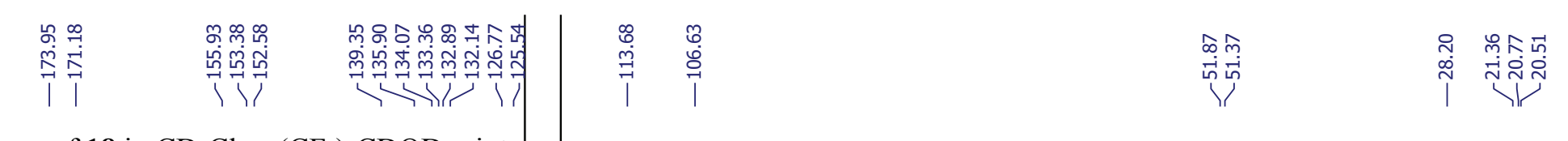

$75 \mathrm{MHz}{ }^{13} \mathrm{C}$ NMR Spectrum of $\mathbf{1 8}$ in $\mathrm{CD}_{2} \mathrm{Cl}_{2}-\left(\mathrm{CF}_{3}\right)_{2} \mathrm{CDOD}$ mixture

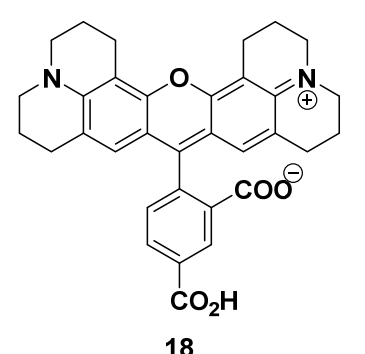

18

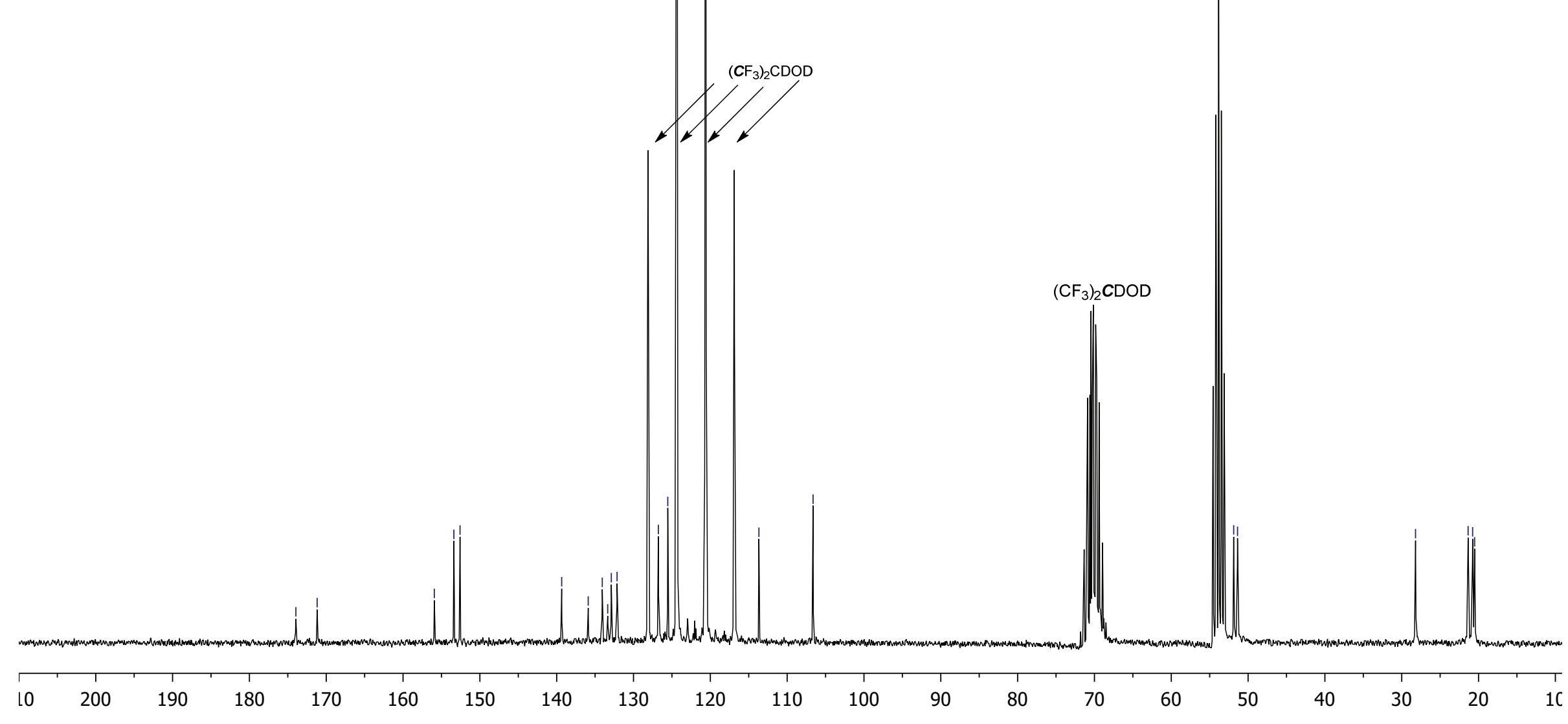




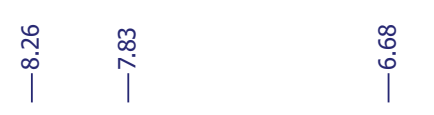

$300 \mathrm{MHz}{ }^{1} \mathrm{H}$ NMR Spectrum of $\mathbf{1 9}$ in $\mathrm{CD}_{2} \mathrm{Cl}_{2}-\left(\mathrm{CF}_{3}\right)_{2} \mathrm{CDOD}$ mixture
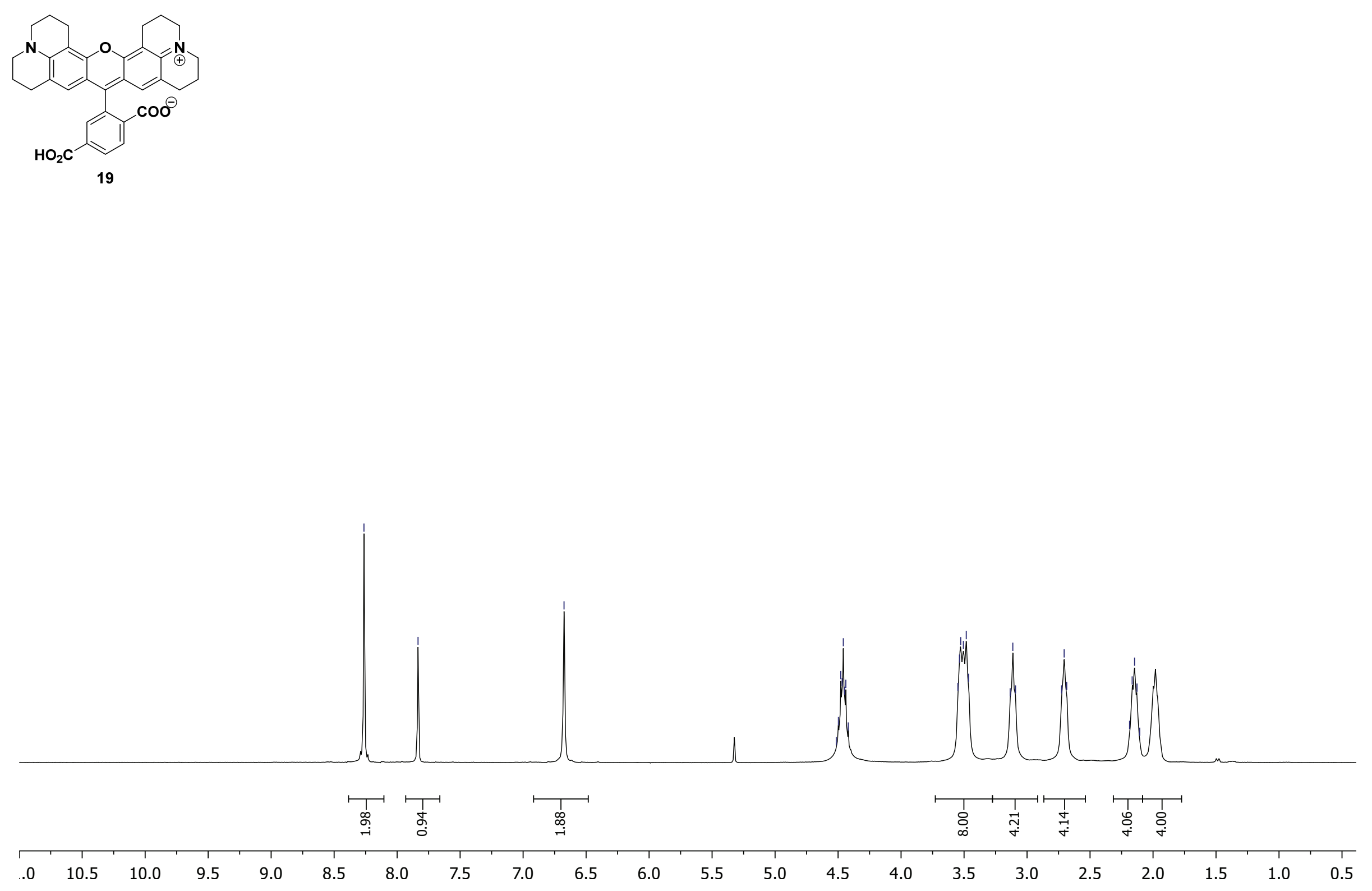


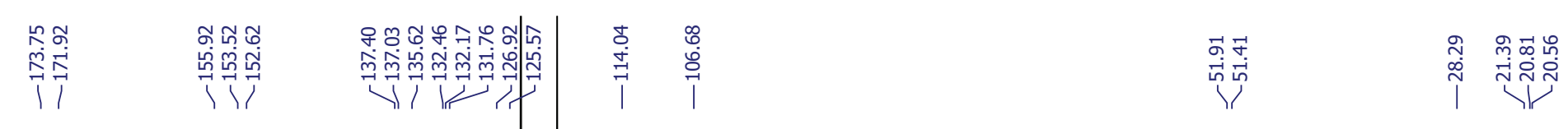

$75 \mathrm{MHz}{ }^{13} \mathrm{C}$ NMR Spectrum of 19 in $\mathrm{CD}_{2} \mathrm{Cl}_{2}-\left(\mathrm{CF}_{3}\right)_{2} \mathrm{CDOD}$ mixture

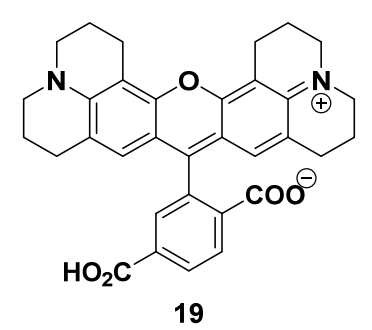

$\left(\mathrm{CF}_{3}\right)_{2} \mathrm{CDOD}$

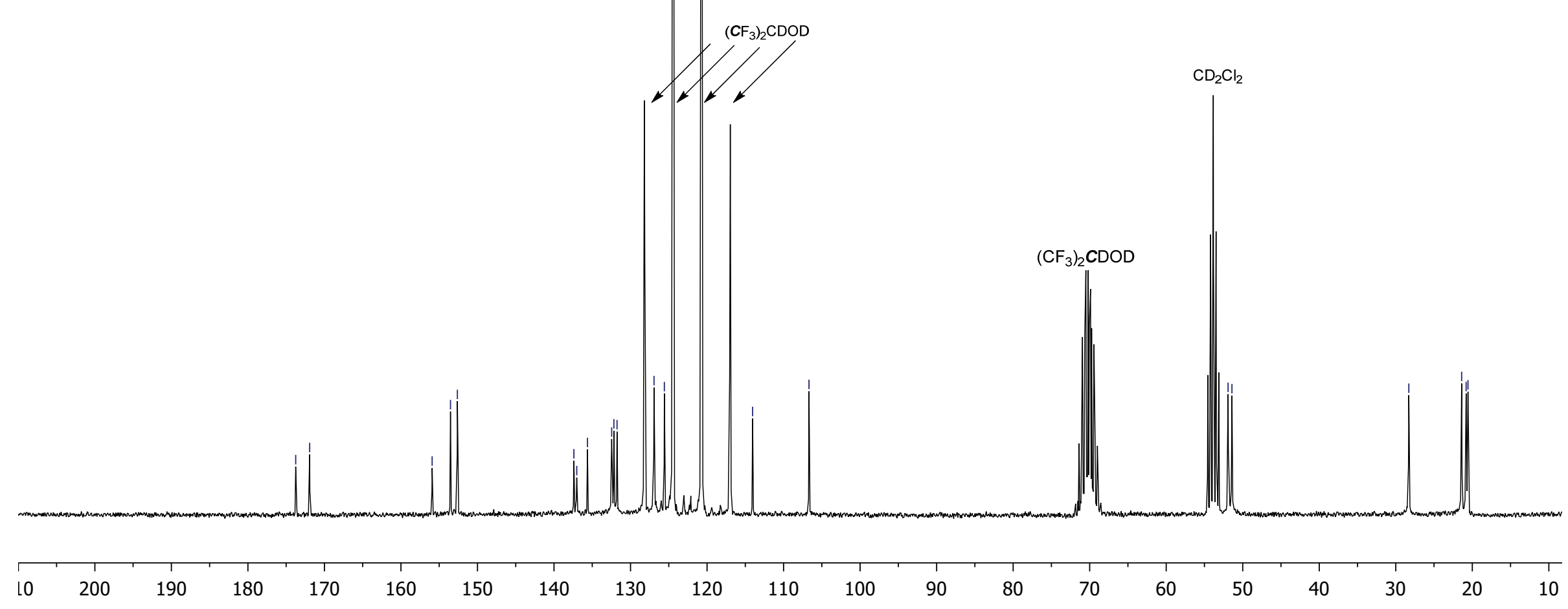


$300 \mathrm{MHz}{ }^{1} \mathrm{H}$ NMR Spectrum of 20 in DMSO- $d_{6}$

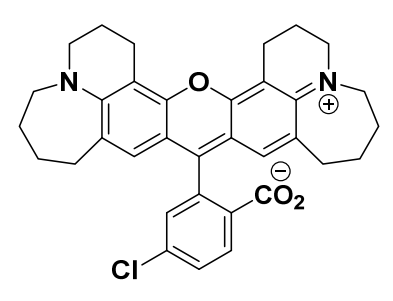

20

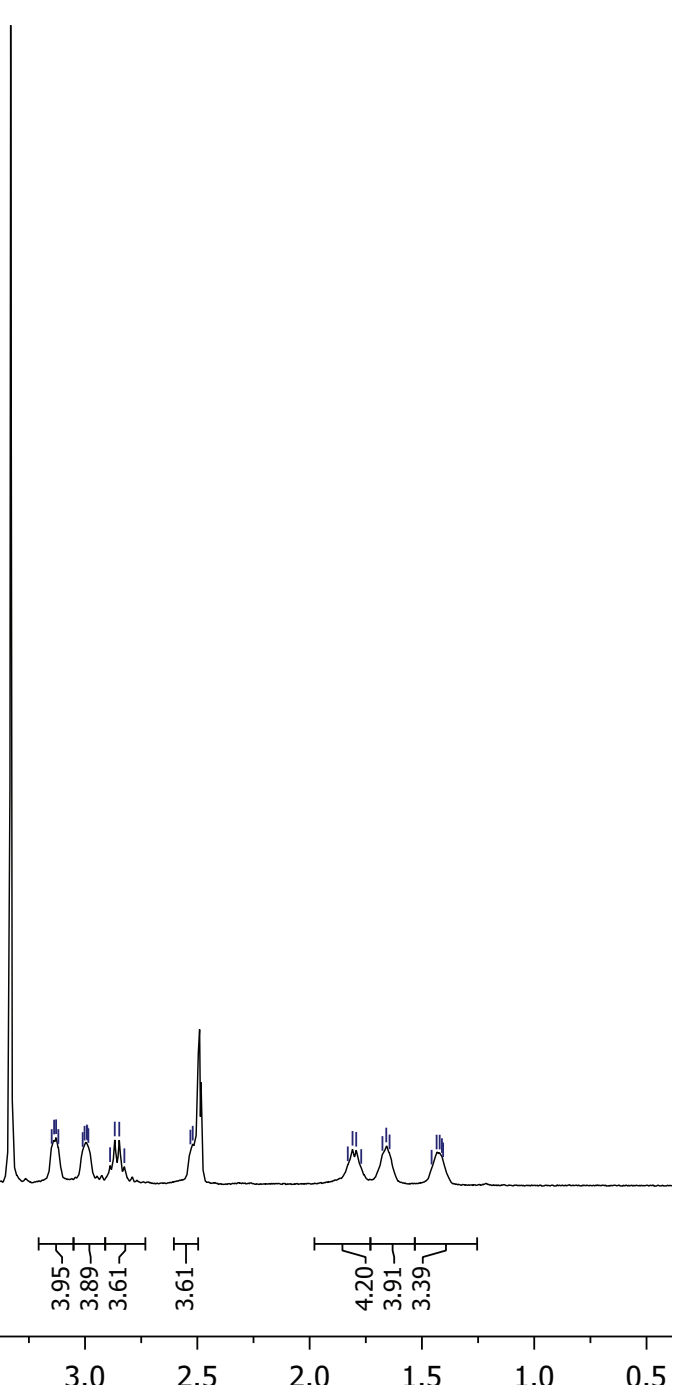




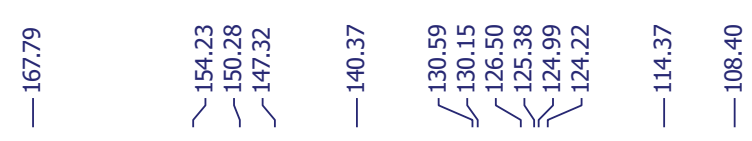

$75 \mathrm{MHz}{ }^{13} \mathrm{C}$ NMR Spectrum of 20 in DMSO- $d_{6}$

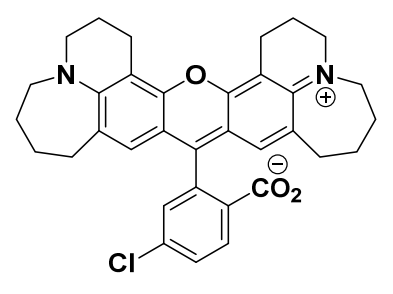

20
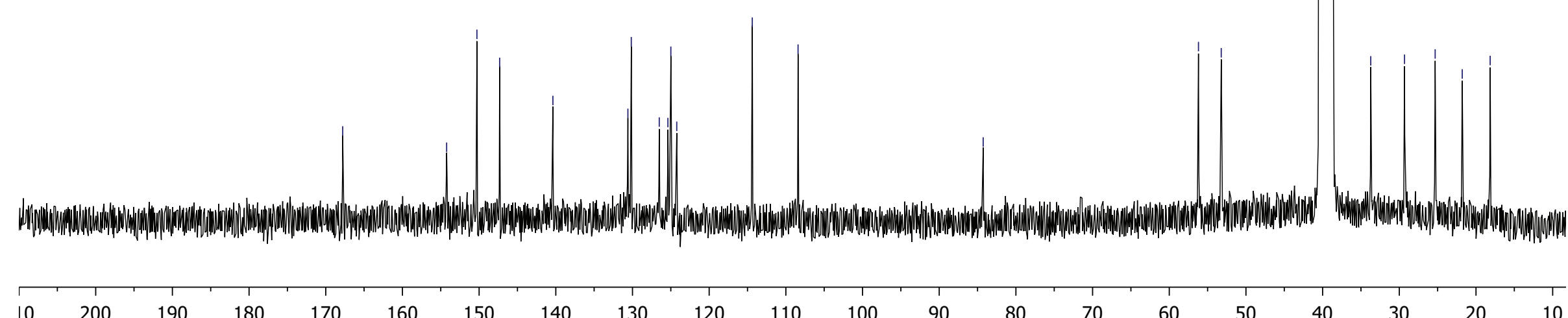

150

140

$130 \quad 120$

110

100

90

80

70

60

50

40

30 
$300 \mathrm{MHz}{ }^{1} \mathrm{H}$ NMR Spectrum of 21 in $\mathrm{CD}_{2} \mathrm{Cl}_{2}$
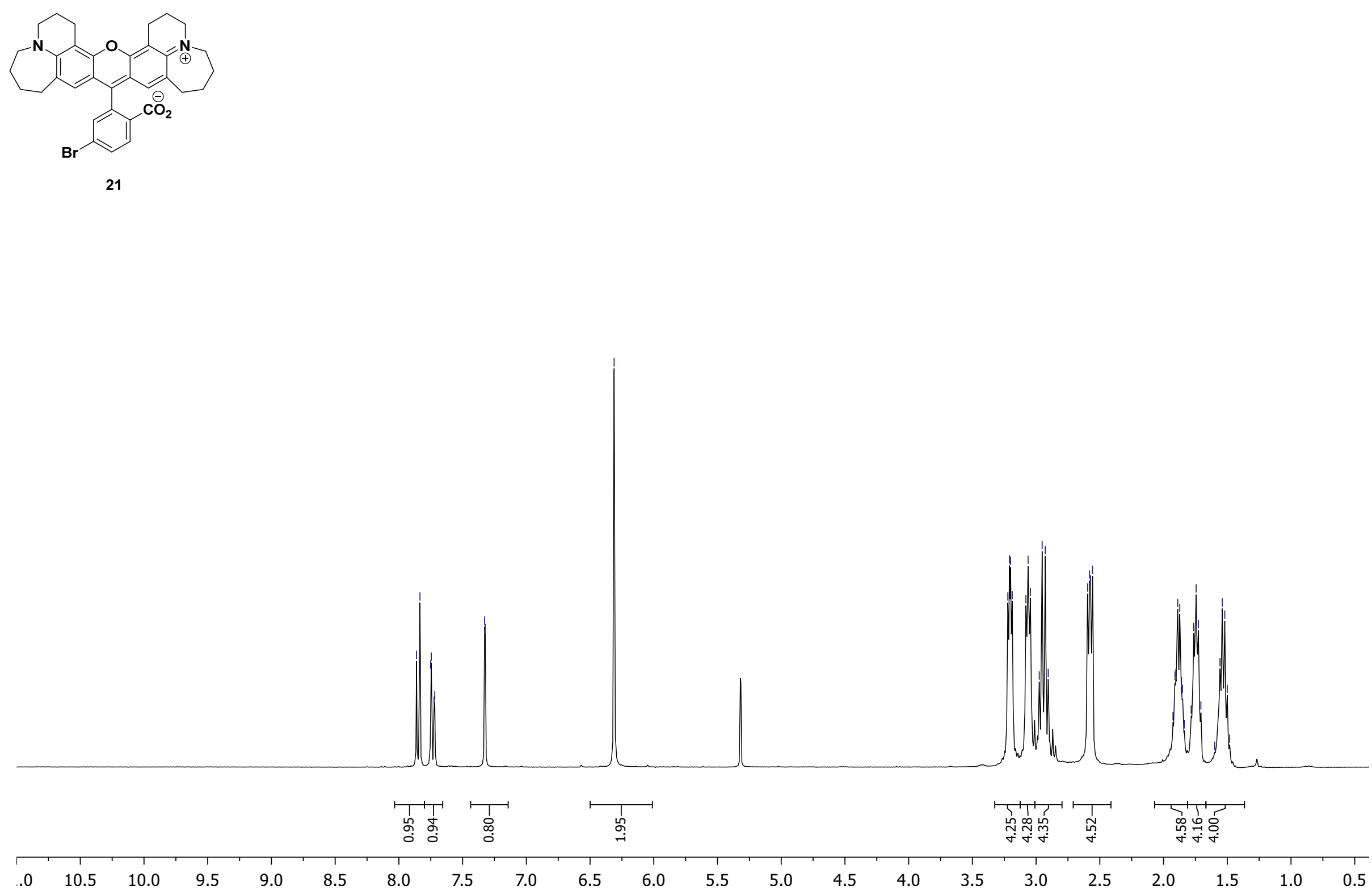


8
0
।

$75 \mathrm{MHz}{ }^{13} \mathrm{C}$ NMR Spectrum of 21 in $\mathrm{CD}_{2} \mathrm{Cl}_{2}$

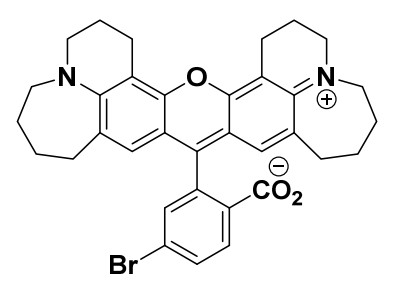

21
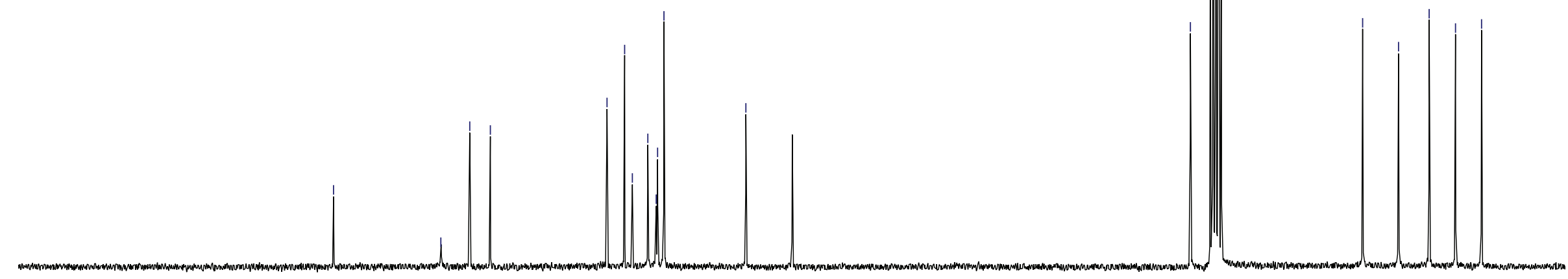

10

200

190

180

170

160

150

140

130

120

110

100

90

80

70

60

50

40

30

20

10 
$400 \mathrm{MHz}{ }^{1} \mathrm{H}$ NMR Spectrum of 24 in DMSO- $d_{6}$

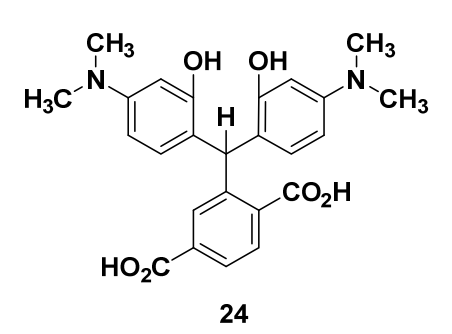

24

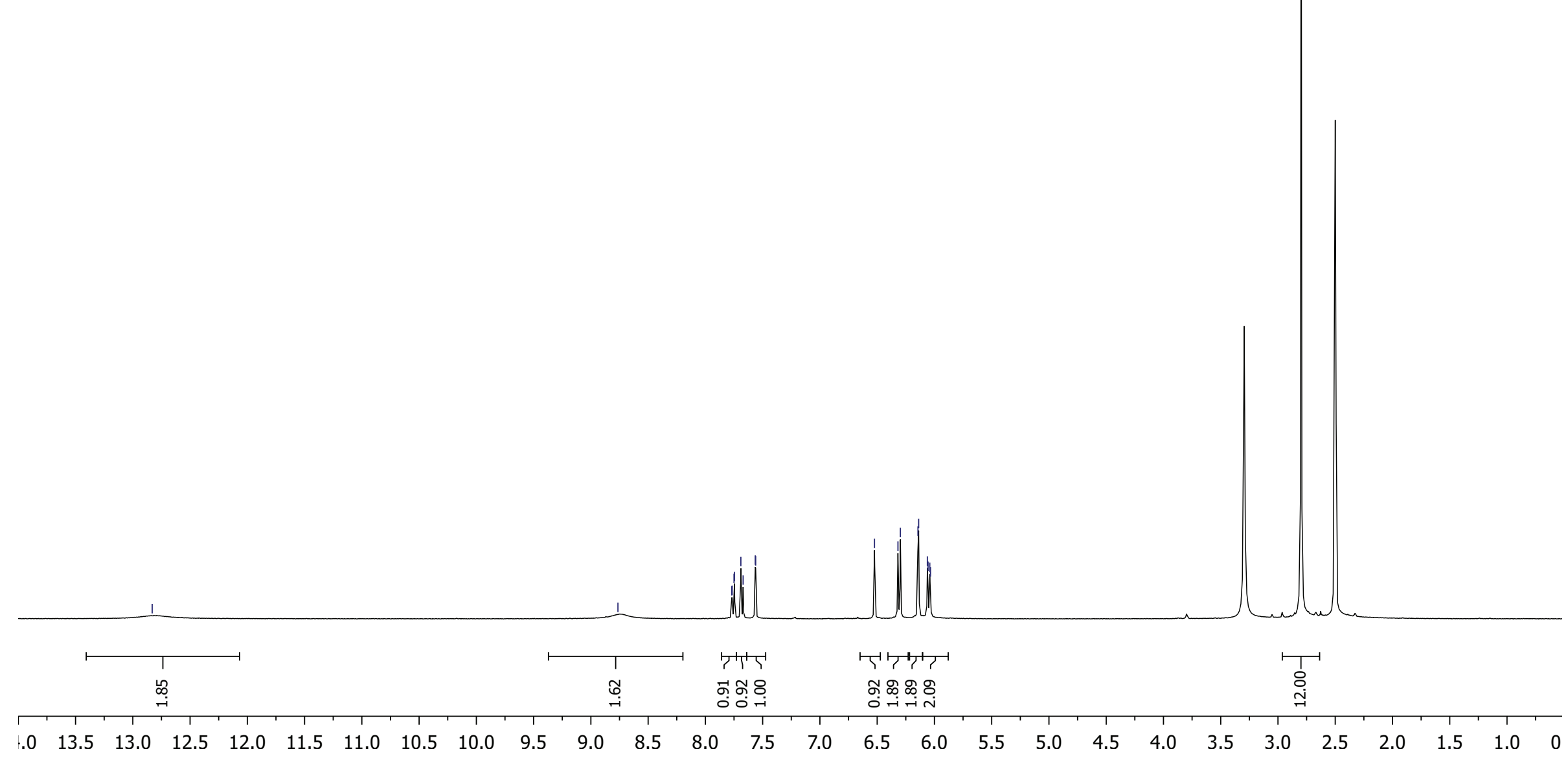




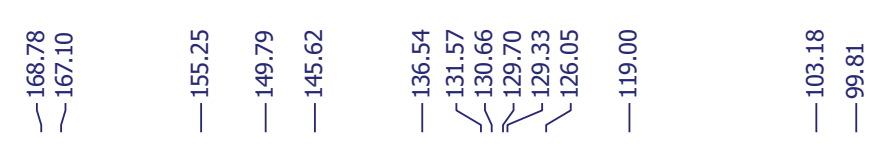

$75 \mathrm{MHz}{ }^{13} \mathrm{C}$ NMR Spectrum of $\mathbf{2 4}$ in DMSO- $d_{6}$
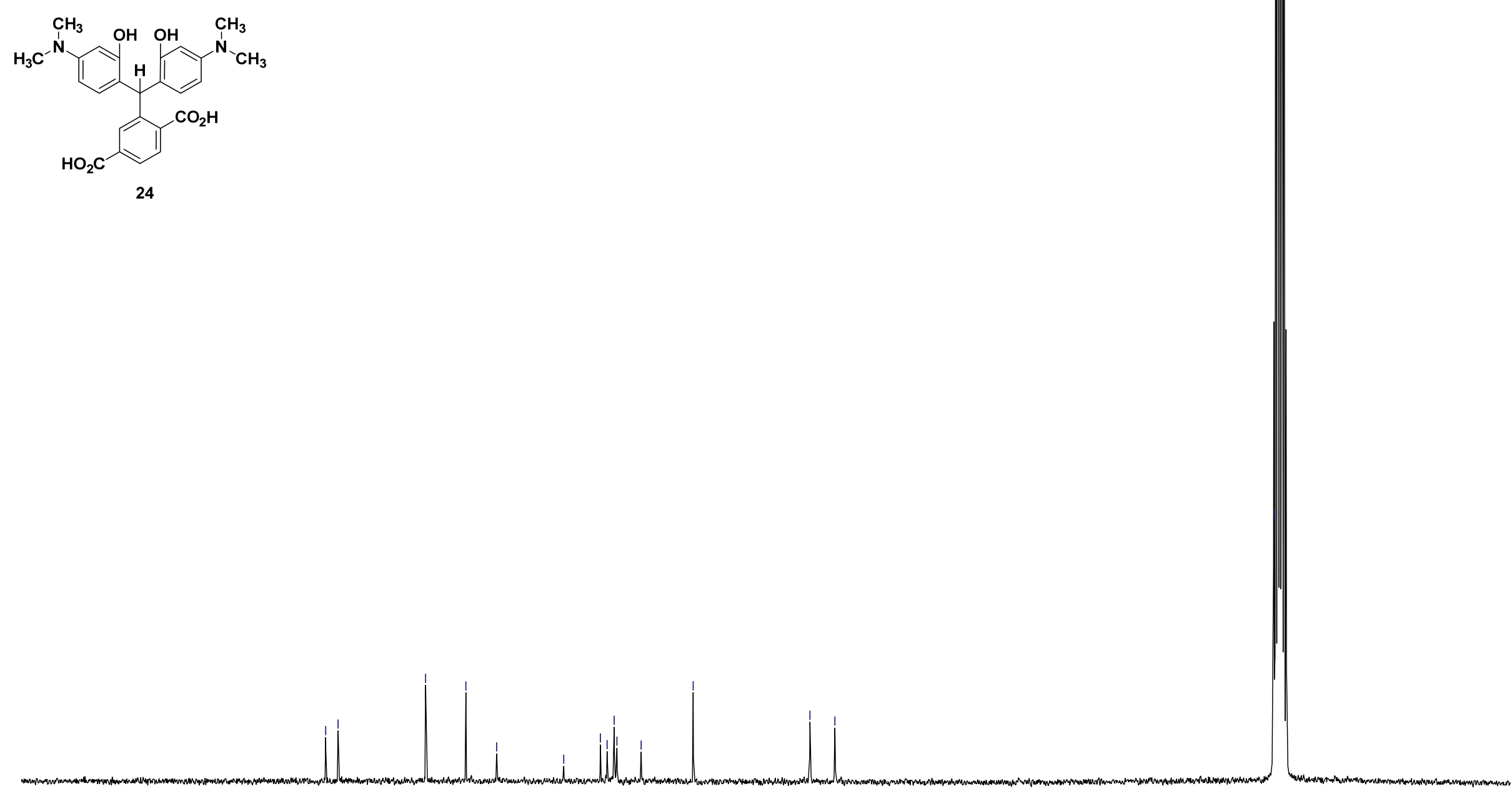

10

200

190

180

170

160

150

140

130

120

110

100

90

80

70

60

50

40 
$300 \mathrm{MHz}{ }^{1} \mathrm{H}$ NMR Spectrum of $\mathbf{S 0 9}$ in DMSO- $d_{6}$
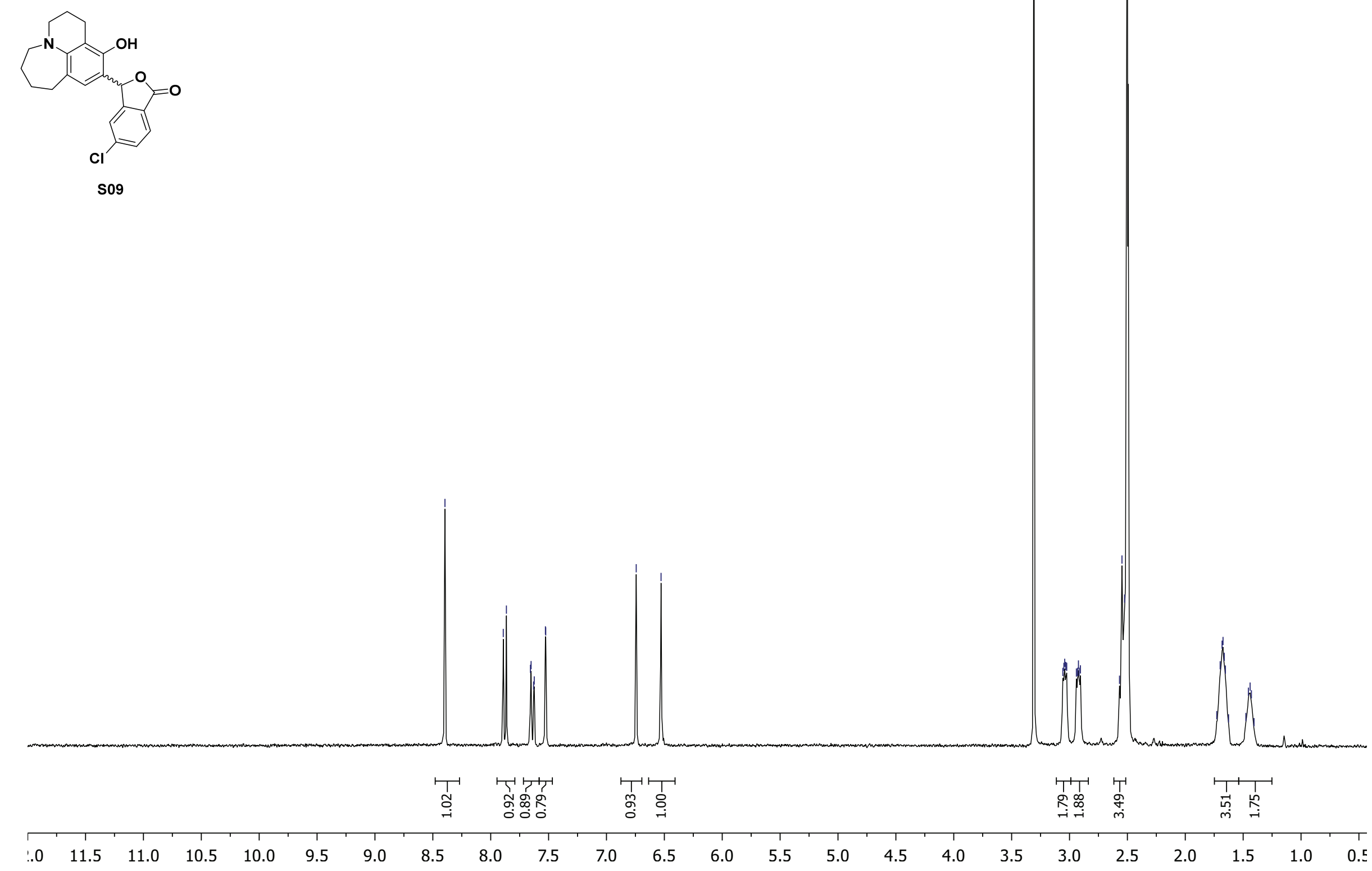


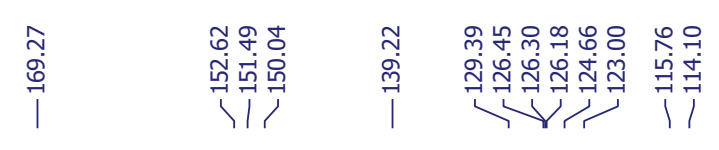

$75 \mathrm{MHz}{ }^{13} \mathrm{C}$ NMR Spectrum of $\mathbf{S 0 9}$ in DMSO- $d_{6}$

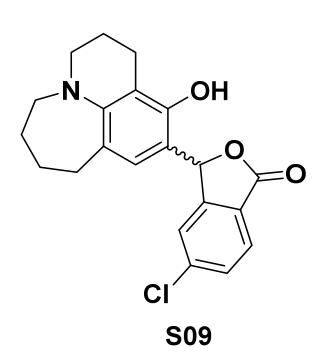

S09
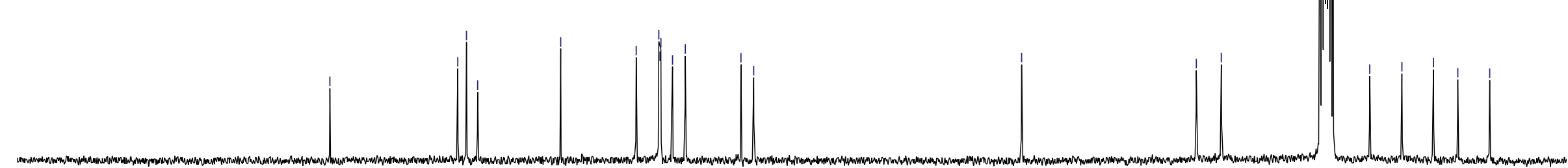

10

200

190

180

170

160

150

140

130

120

110

100

90

80

70

60

50

40

30

20

10 
$300 \mathrm{MHz}{ }^{1} \mathrm{H}$ NMR Spectrum of $\mathbf{S 1 0}$ in DMSO- $d_{6}$
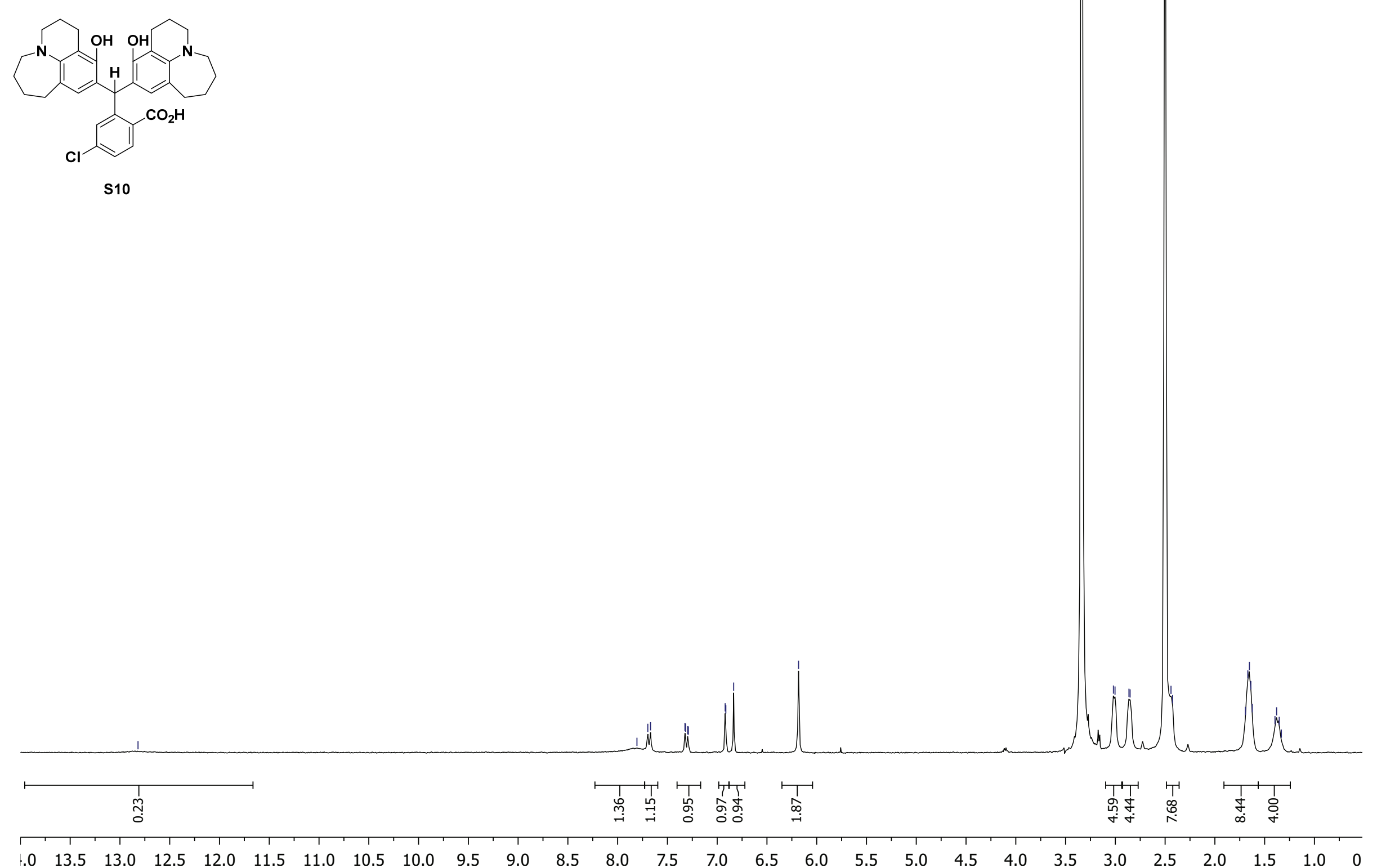
$75 \mathrm{MHz}{ }^{13} \mathrm{C}$ NMR Spectrum of $\mathbf{S 1 0}$ in DMSO- $d_{6}$
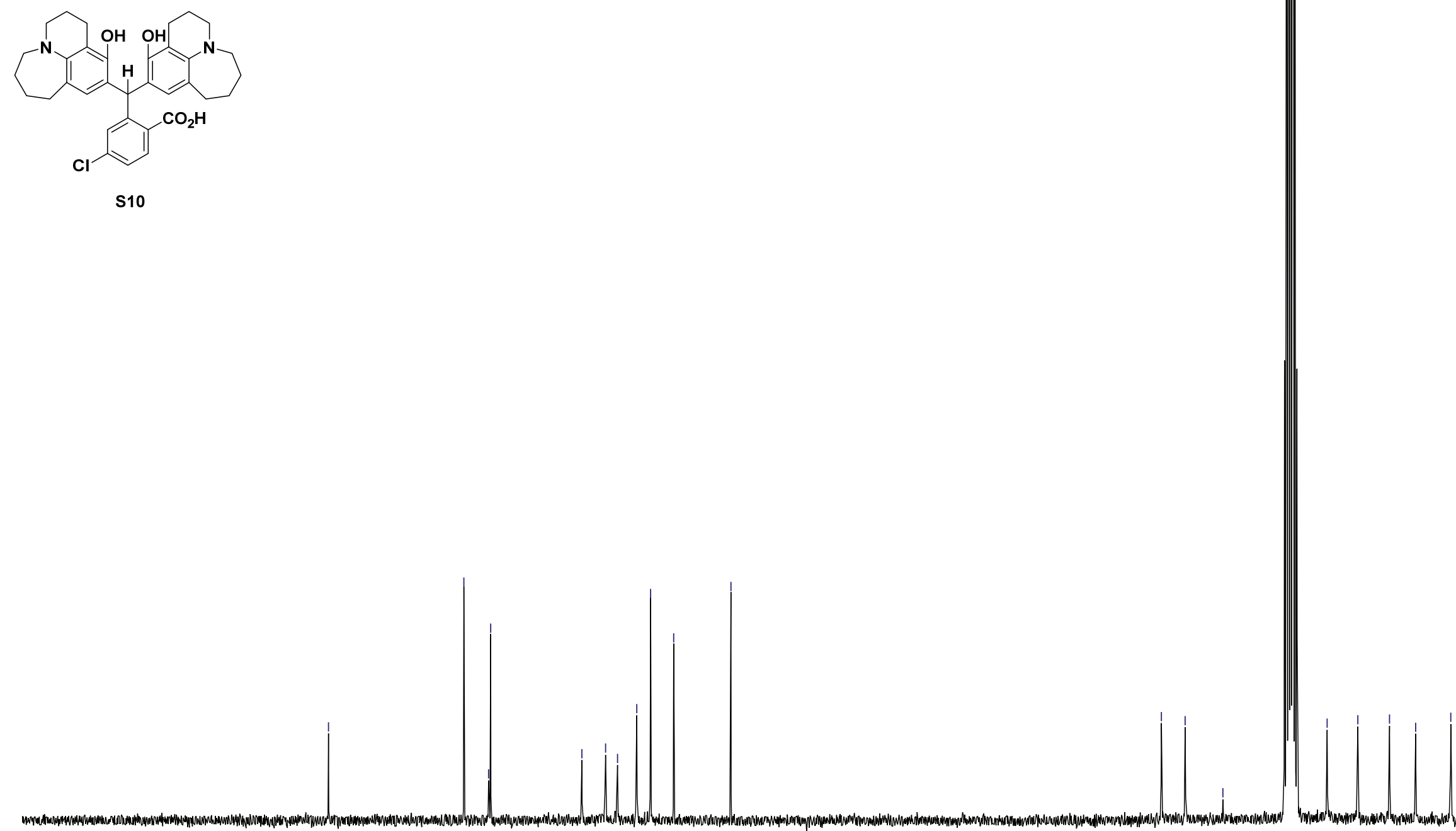

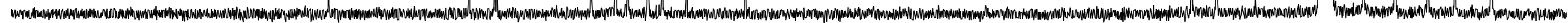

10

200

190

180

170

160

150

140

130

120

110

100

90

80

70

60

50

40

30

20 
$300 \mathrm{MHz}{ }^{1} \mathrm{H}$ NMR Spectrum of S11 in DMSO- $d_{6}$

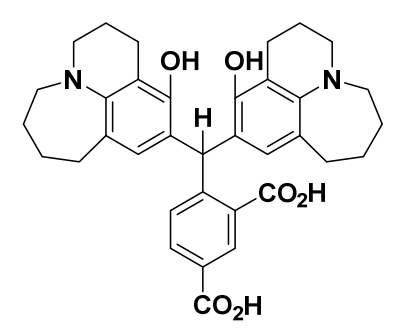

$\mathrm{s} 11$

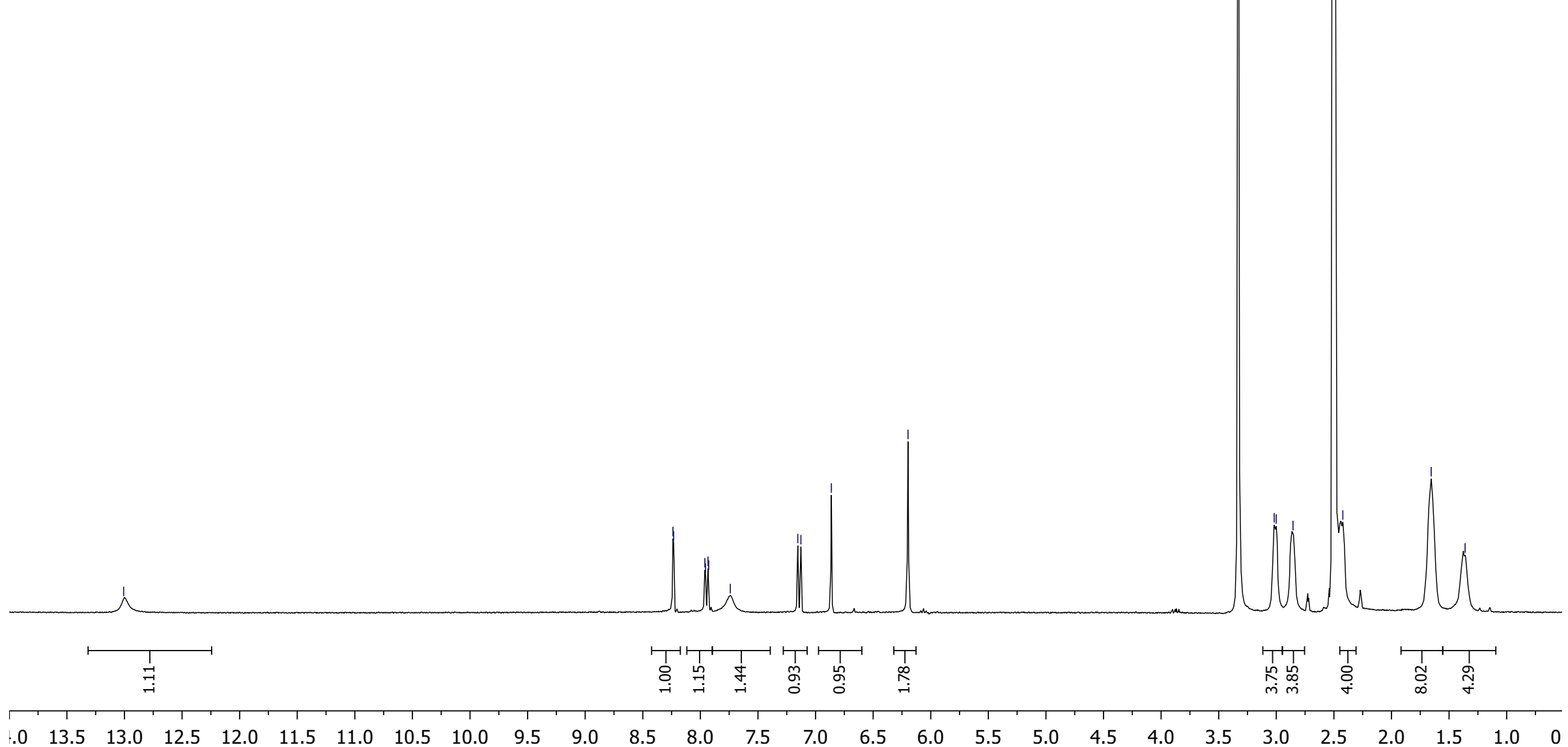




$$
\text { OR }
$$

$75 \mathrm{MHz}{ }^{13} \mathrm{C}$ NMR Spectrum of $\mathbf{S 1 1}$ in DMSO- $d_{6}$

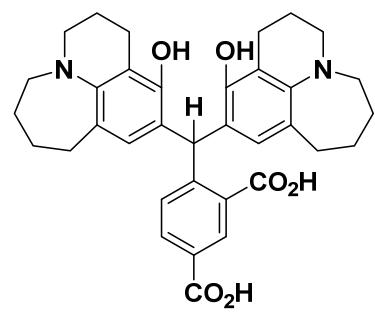

S11

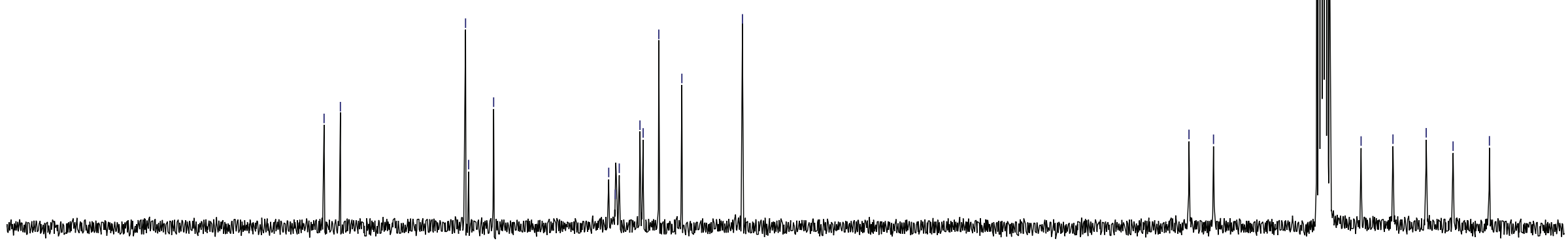




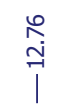

$300 \mathrm{MHz}{ }^{1} \mathrm{H}$ NMR Spectrum of $\mathbf{S 1 2}$ in DMSO- $d_{6}$

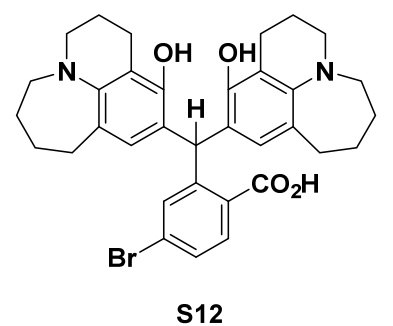

S12

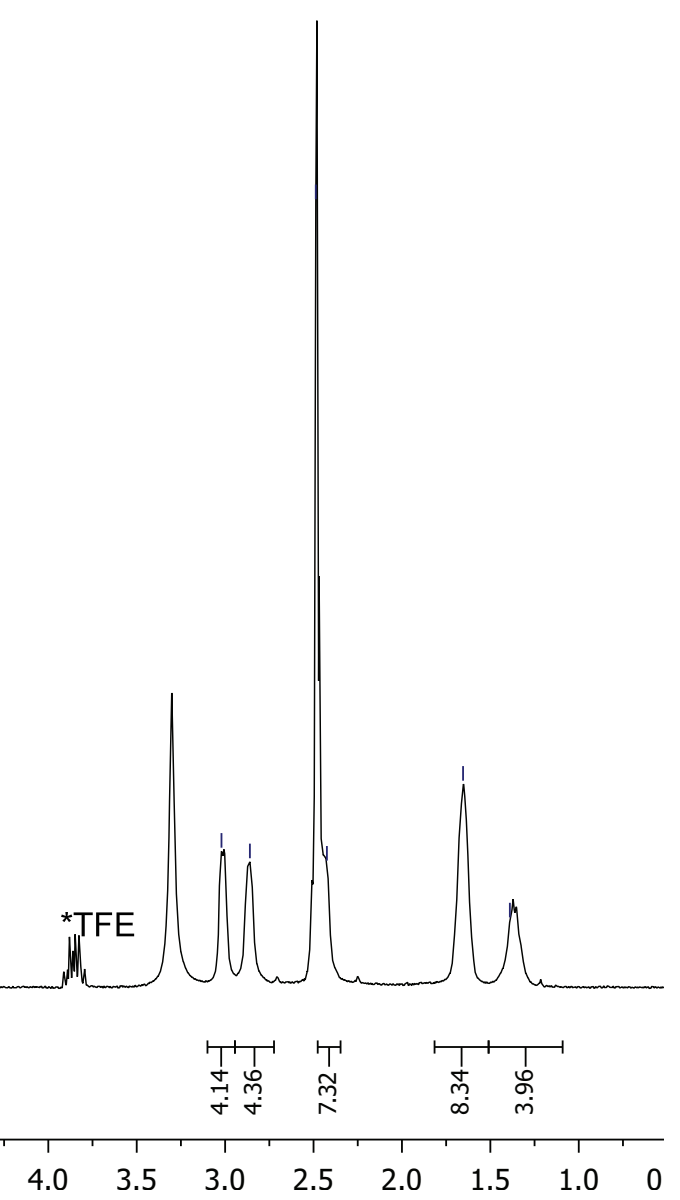


$75 \mathrm{MHz}{ }^{13} \mathrm{C}$ NMR Spectrum of $\mathbf{S 1 2}$ in DMSO- $d_{6}$
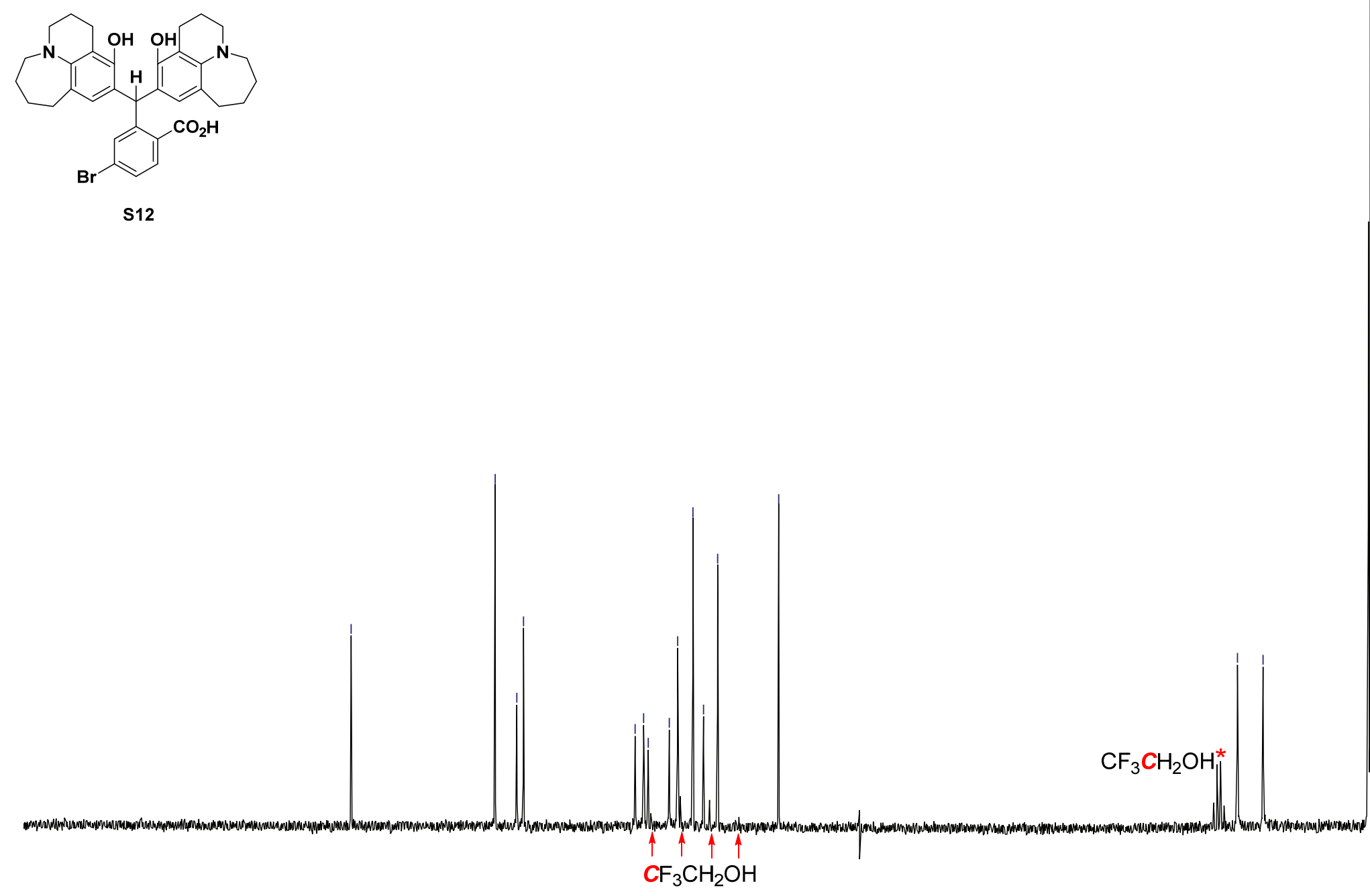

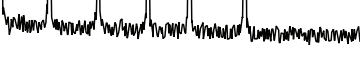
$\mathrm{CF}_{3} \mathrm{CH}_{2} \mathrm{OH}$ 